Supporting Information:

Steric Effects in the Aerobic Oxidation of $\pi$-Allylnickel(II) Complexes with N-Heterocyclic

\title{
Carbenes
}

Benjamin R. Dible and Matthew S. Sigman

Figure S-1. ${ }^{1} \mathrm{H}-\mathrm{NMR}$ spectrum of SIiPr-allylNiCl (1b) S-2

Figure S-2. ${ }^{1} \mathrm{H}-\mathrm{NMR}$ spectrum of IMes-allylNiCl (1c) S-3

Figure S-3. ${ }^{1} \mathrm{H}-\mathrm{NMR}$ spectrum of IBioxMe${ }_{4}$-allylNiCl (1f) S-4

Figure S-4. ${ }^{1} \mathrm{H}-\mathrm{NMR}$ spectrum of ICy-allylNiCl (1g) S-5

Figure S-5. ${ }^{1} \mathrm{H}-\mathrm{NMR}$ spectrum of ITol-allylNiCl (1h) S-6

Figure S-6. ${ }^{1} \mathrm{H}-\mathrm{NMR}$ spectrum of MeItBu-HI $\quad$ S-7

Figure S-7. ${ }^{1} \mathrm{H}-\mathrm{NMR}$ spectrum of MeItBu-allylNiCl (1i) S-8

Figure S-8. ${ }^{1} \mathrm{H}-\mathrm{NMR}$ spectrum of MeIiPr-HI S-9

Figure S-9. ${ }^{1} \mathrm{H}-\mathrm{NMR}$ spectrum of MeIiPr-allylNiCl (1j) S-10

Figure S-10. ${ }^{1} \mathrm{H}$-NMR spectrum of IMe-allylNiCl (1k) S-11

Figure S-11. ${ }^{1} \mathrm{H}-\mathrm{NMR}$ spectrum of MeItBu-allylNiI (1I) S-12

Figure S-12. VT-NMR spectra of IBioxMe 4 -allylNiCl (1f) in $\mathrm{C}_{6} \mathrm{D}_{6} \quad$ S-13

$\begin{array}{ll}\text { Figure S-13. VT-NMR spectra of ICy-allylNiCl (1g) in } \mathrm{C}_{6} \mathrm{D}_{6} & \text { S-14 }\end{array}$

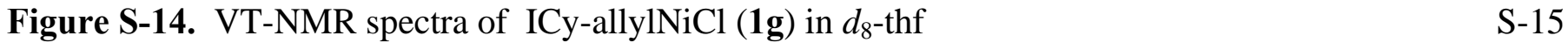

Figure S-15. VT-NMR spectra of ITol-allylNiCl (1h) in $\mathrm{C}_{6} \mathrm{D}_{6} \quad$ S-16

$\begin{array}{ll}\text { Figure S-16. VT-NMR spectra of ITol-allylNiCl (1h) in } d_{8} \text {-thf } & \text { S-17 }\end{array}$

Figure S-17. ORTEP of ItBu-allylNiCl (1d) S-18

Figure S-18. ORTEP of IBioxMe $\mathrm{A}_{4}$-allylNiCl (1f) S-19

$\begin{array}{ll}\text { Figure S-19. ORTEP of MeItBu-allylNiI (1I) S-20 } & \end{array}$

Figure S-20. ORTEP of trans-[ITol $\left.{ }_{2} \mathrm{NiCl}_{2}\right](\mathbf{3 h}) \quad \mathrm{S}-21$

Crystallographic Details. ItBu-allylNiCl (1d), IBioxMe 4 -allylNiCl (1f), S-22

MeItBu-allylNiI (11), trans-[ITol $\left.{ }_{2} \mathrm{NiCl}_{2}\right]$ (3h)

Tables S-1 to S-6. Tables of Crystallographic Data for ItBu-allylNiCl (1d) S-26

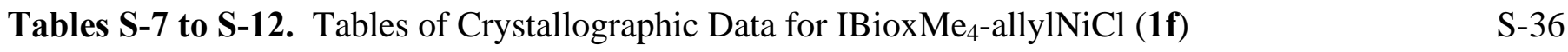

Tables S-13 to S-18. Tables of Crystallographic Data for MeItBu-allylNiI (11) S-53

Tables S-19 to S-24. Tables of Crystallographic Data for trans-[$\left[\mathrm{ITol}_{2} \mathrm{NiCl}_{2}\right](\mathbf{3 h}) \quad$ S-64 
Figure S-1. ${ }^{1} \mathrm{H}-\mathrm{NMR}$ spectrum of SliPr-allyINiCl (1b)

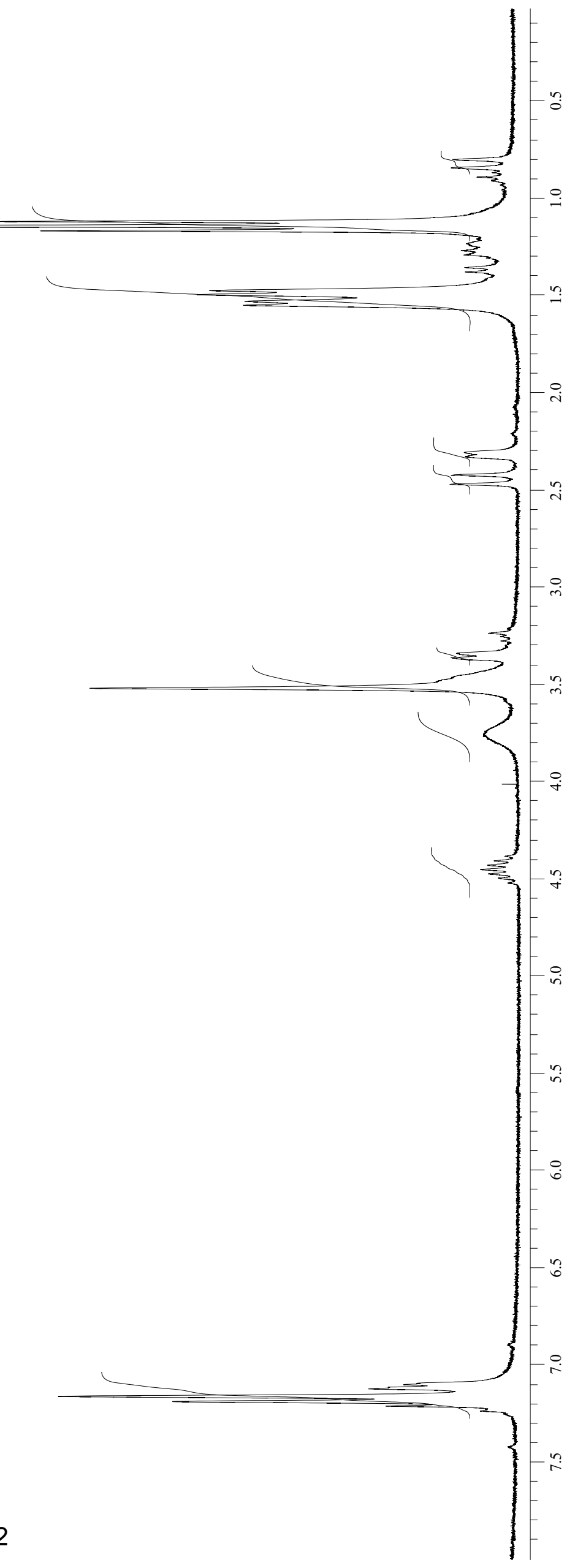


Figure S-2. ${ }^{1} \mathrm{H}-\mathrm{NMR}$ spectrum of IMes-allylNiCl (1c)

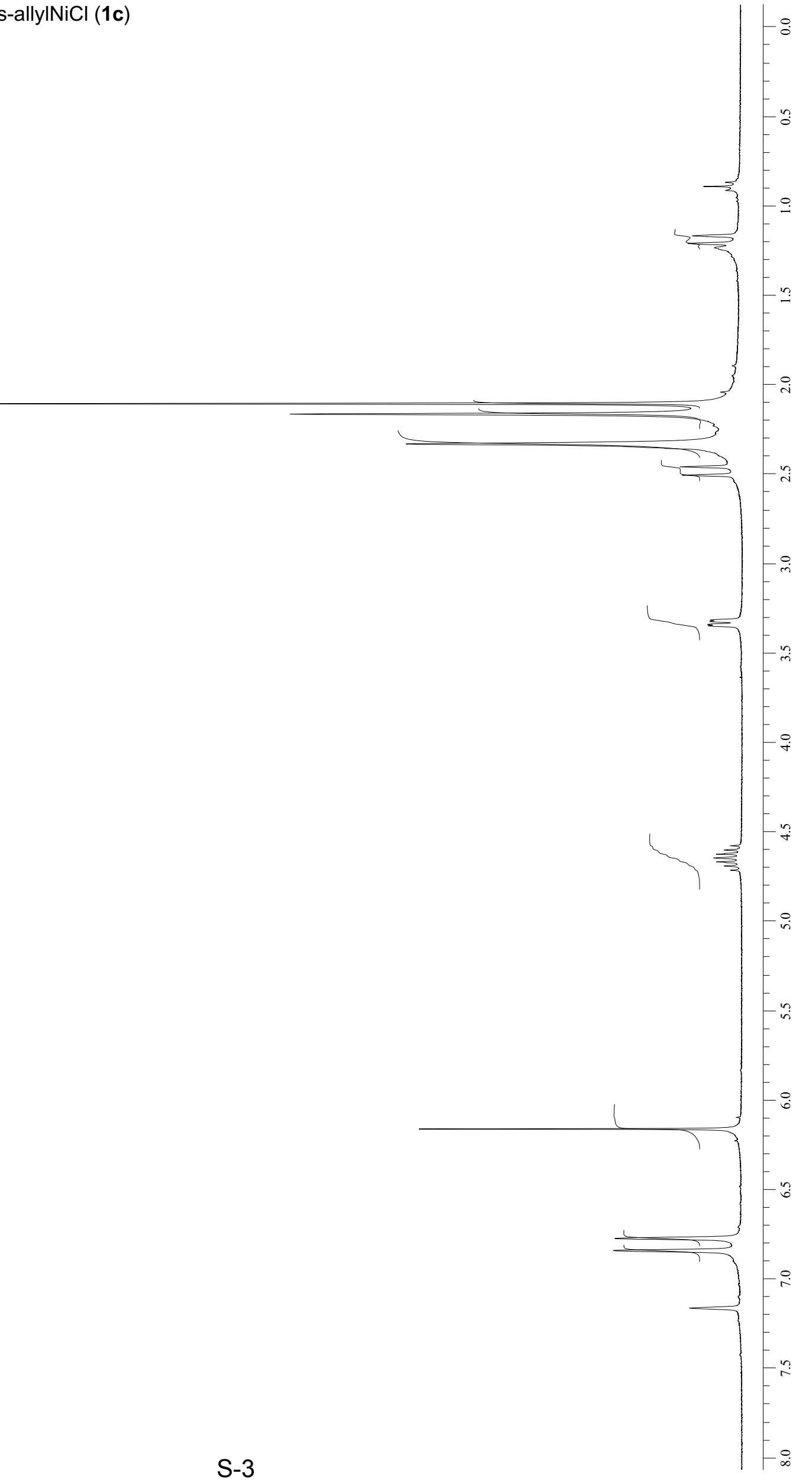


Figure S-3. ${ }^{1} \mathrm{H}-\mathrm{NMR}$ spectrum of IBioxMe $\mathrm{A}_{4}$-allyINiCl (1f)

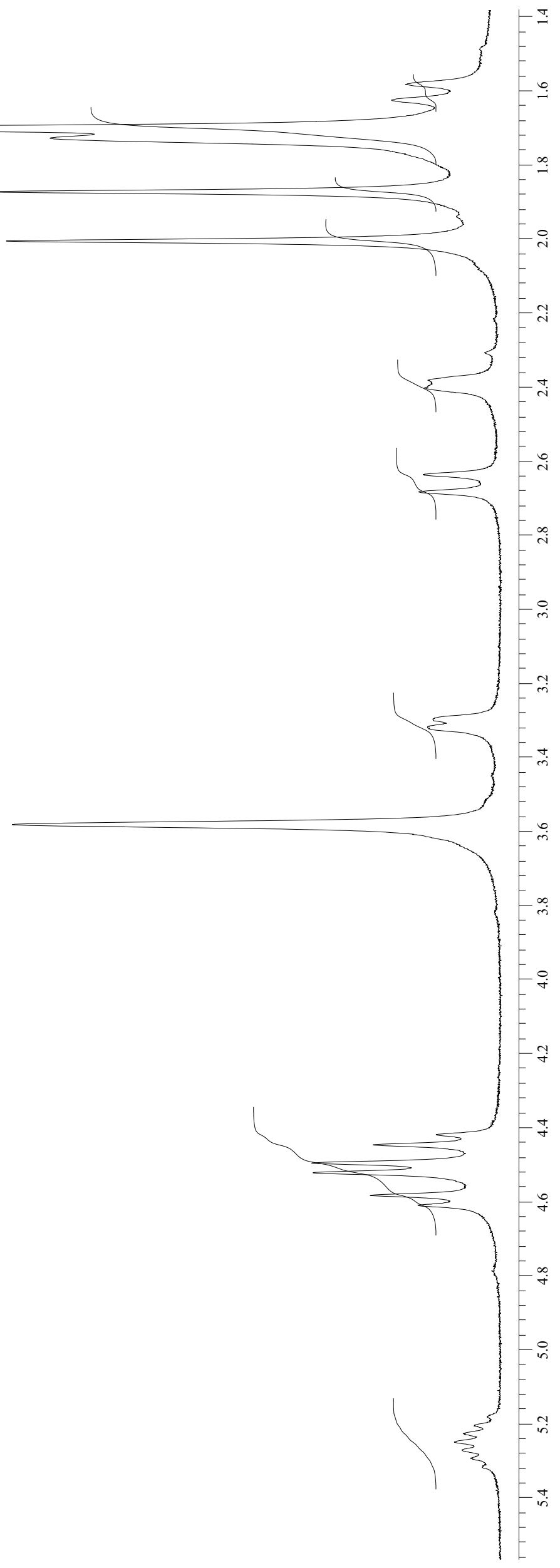


Figure S-4. ${ }^{1} \mathrm{H}-\mathrm{NMR}$ spectrum of ICy-allyINiCl (19)

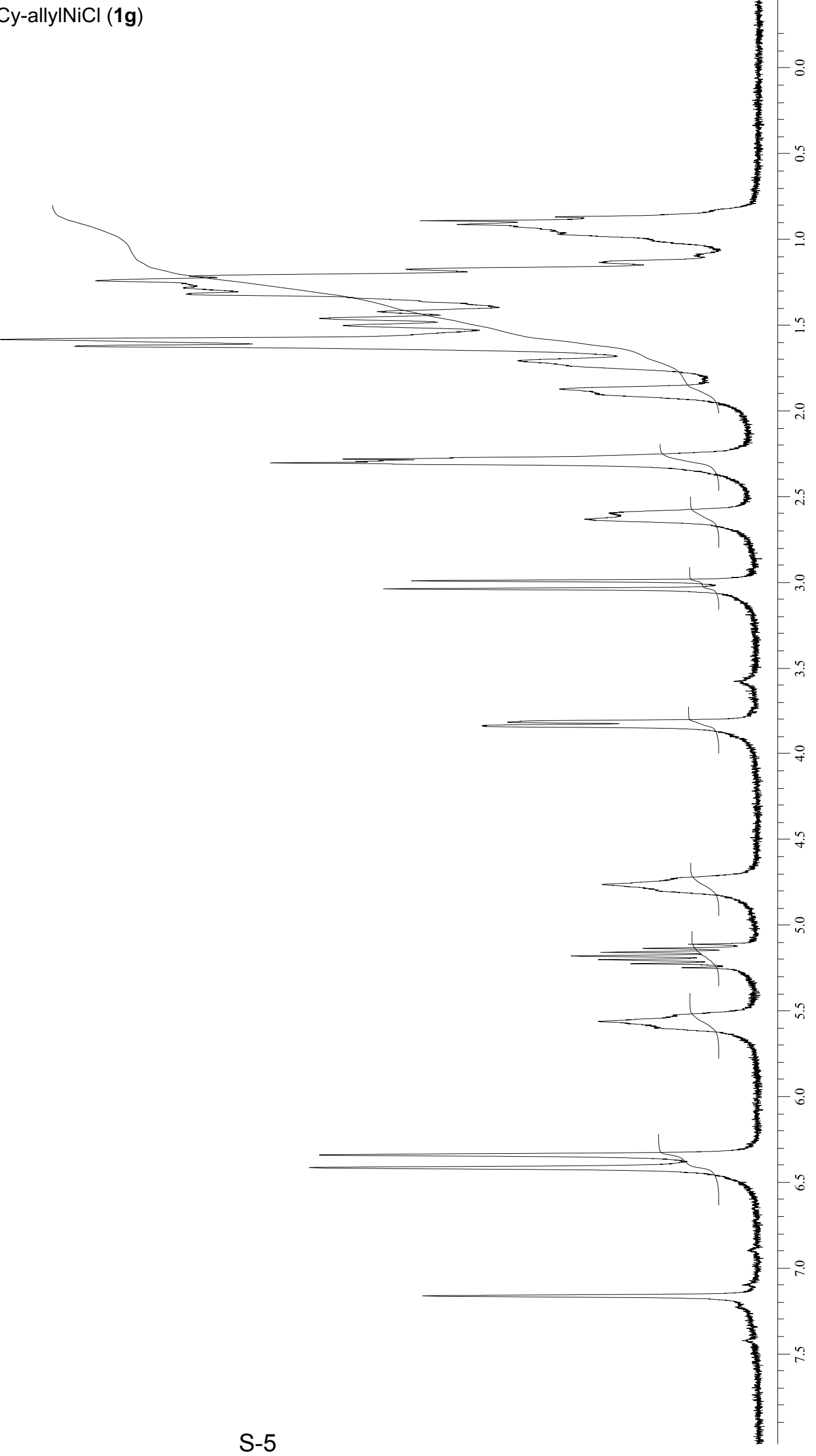


Figure S-5. ${ }^{1} \mathrm{H}-\mathrm{NMR}$ spectrum of ITol-allyINiCl (1h)
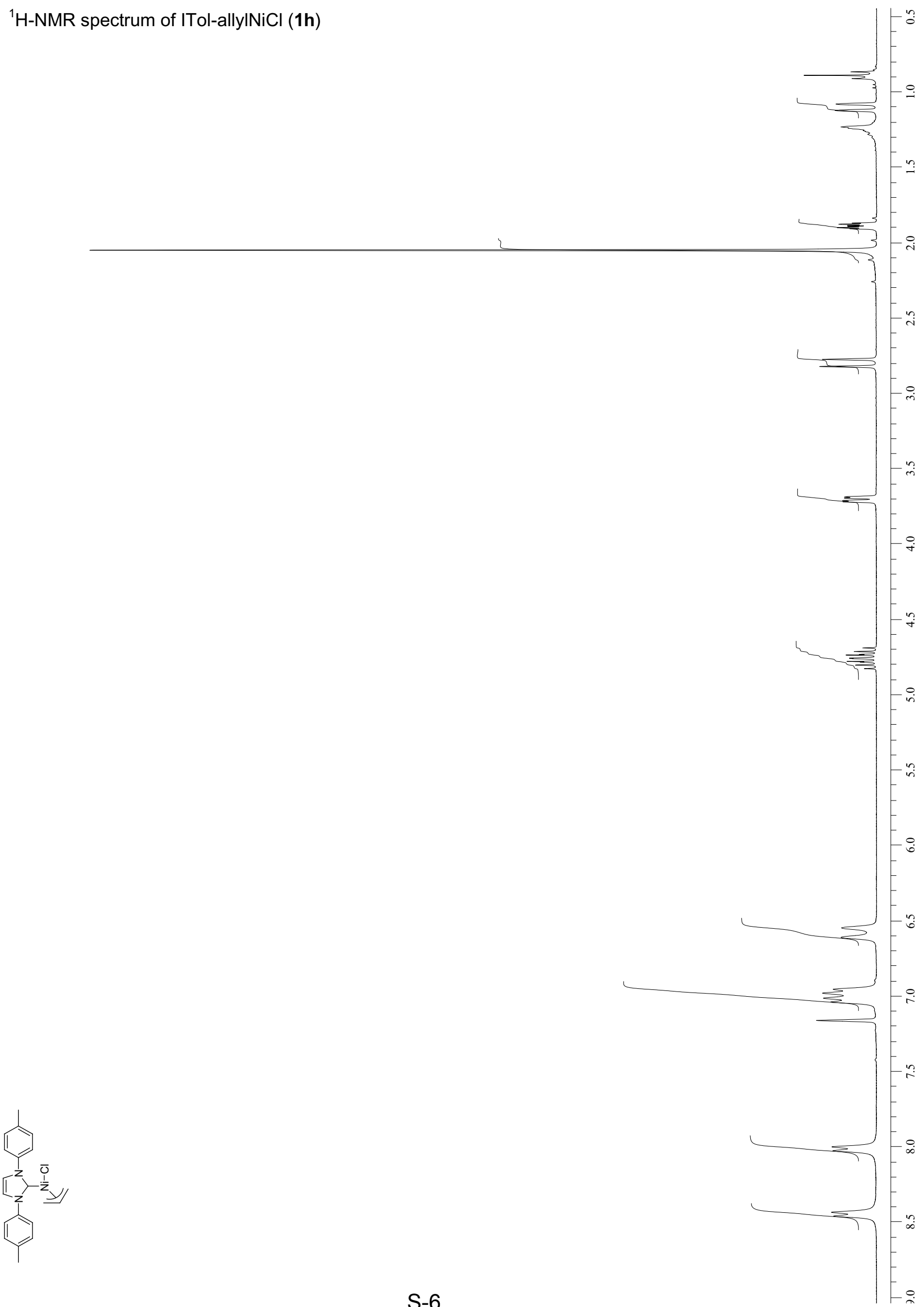
Figure S-6. ${ }^{1} \mathrm{H}-\mathrm{NMR}$ spectrum of MeltBu-HI
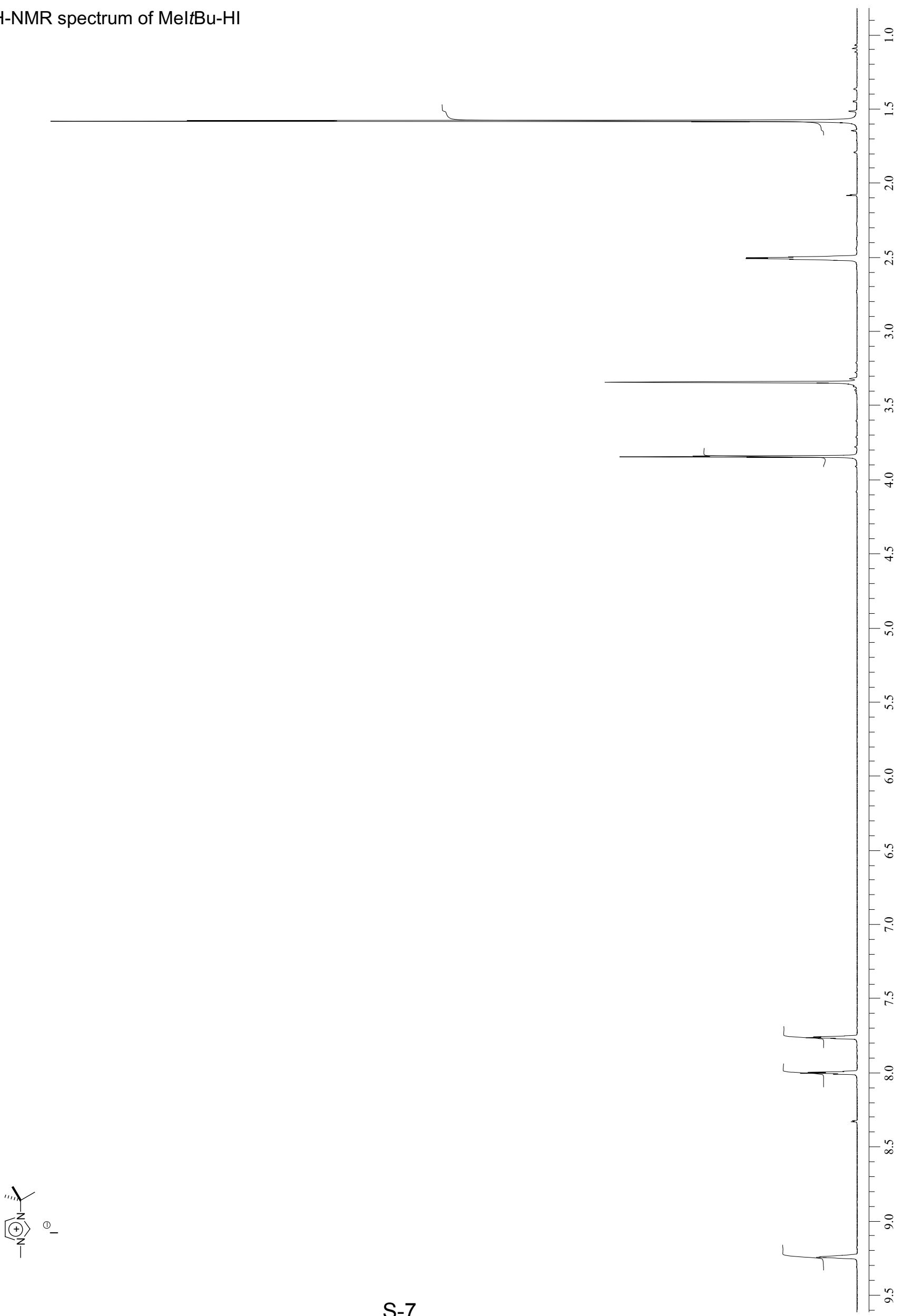
Figure S-7. ${ }^{1} \mathrm{H}-\mathrm{NMR}$ spectrum of MeltBu-allyINiCl (1i)

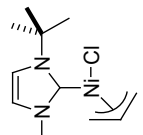


Figure S-8. ${ }^{1} \mathrm{H}-\mathrm{NMR}$ spectrum of MeliPr-HI

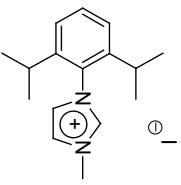


Figure S-9. ${ }^{1} \mathrm{H}-\mathrm{NMR}$ spectrum of MeliPr-allylNiCl (1j)
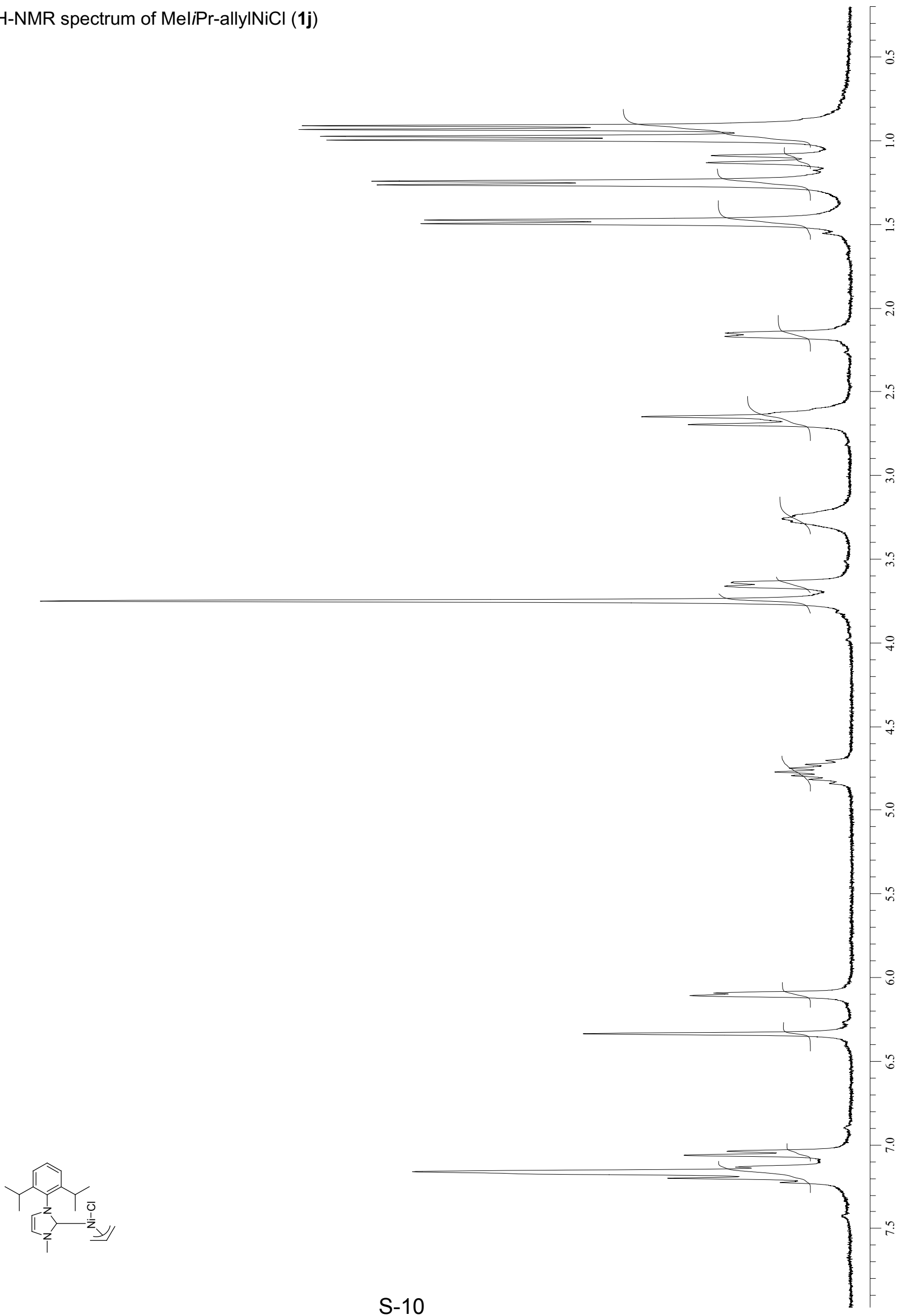
Figure S-10. ${ }^{1} \mathrm{H}-\mathrm{NMR}$ spectrum of IMe-allyINiCl (1k)

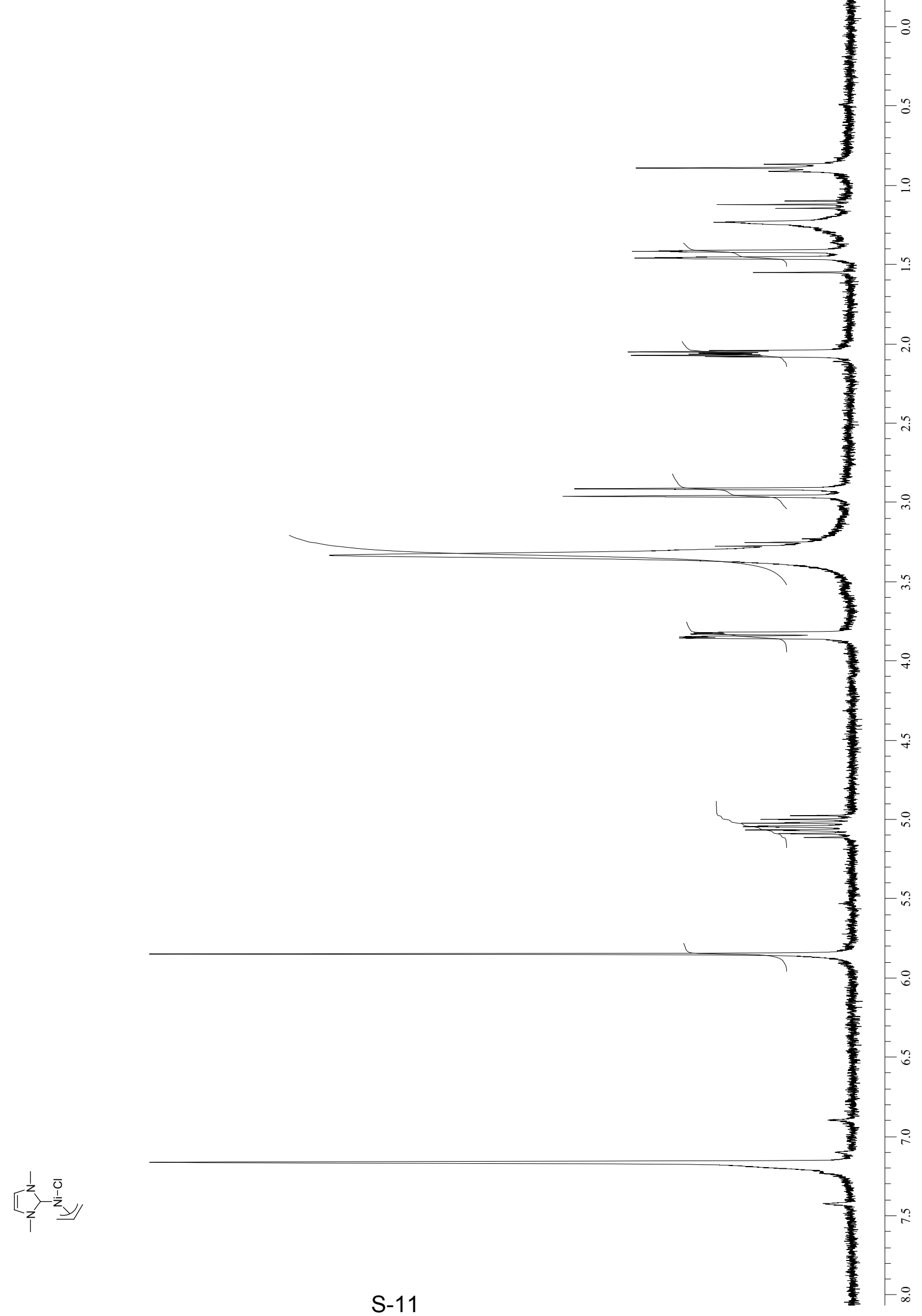


Figure S-11. 'H-NMR spectrum of MeltBu-allyINil (1I)

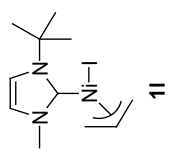




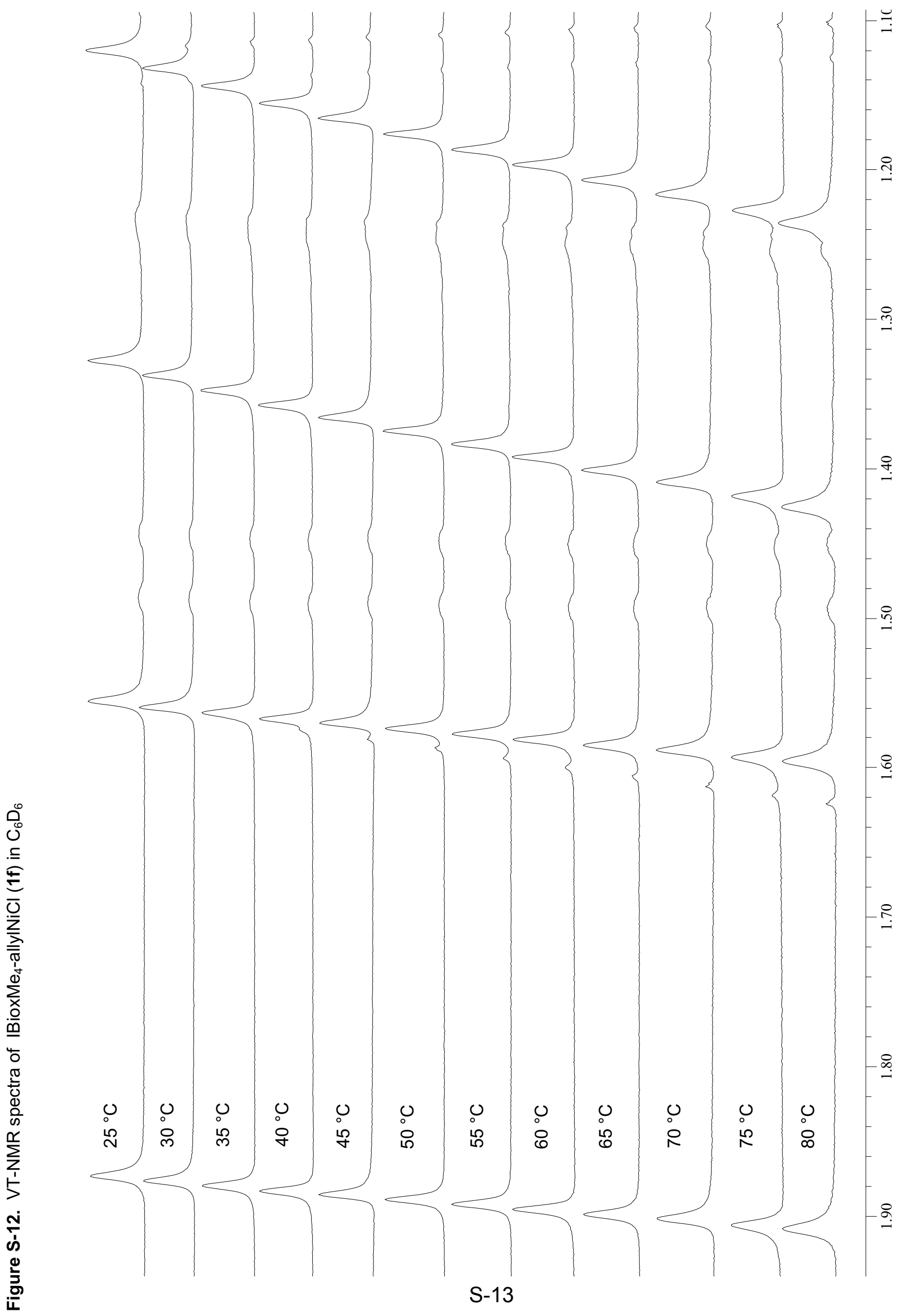




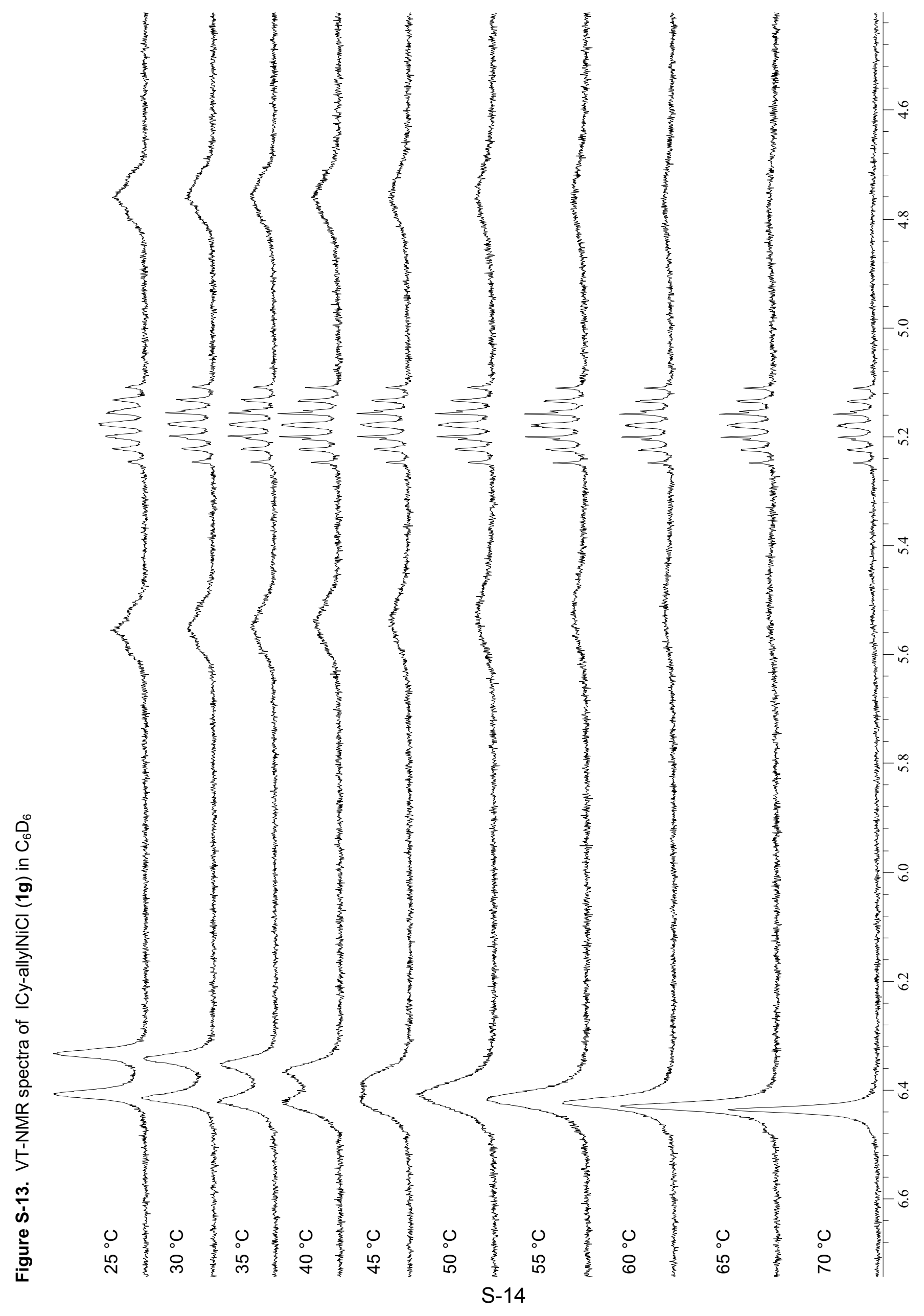




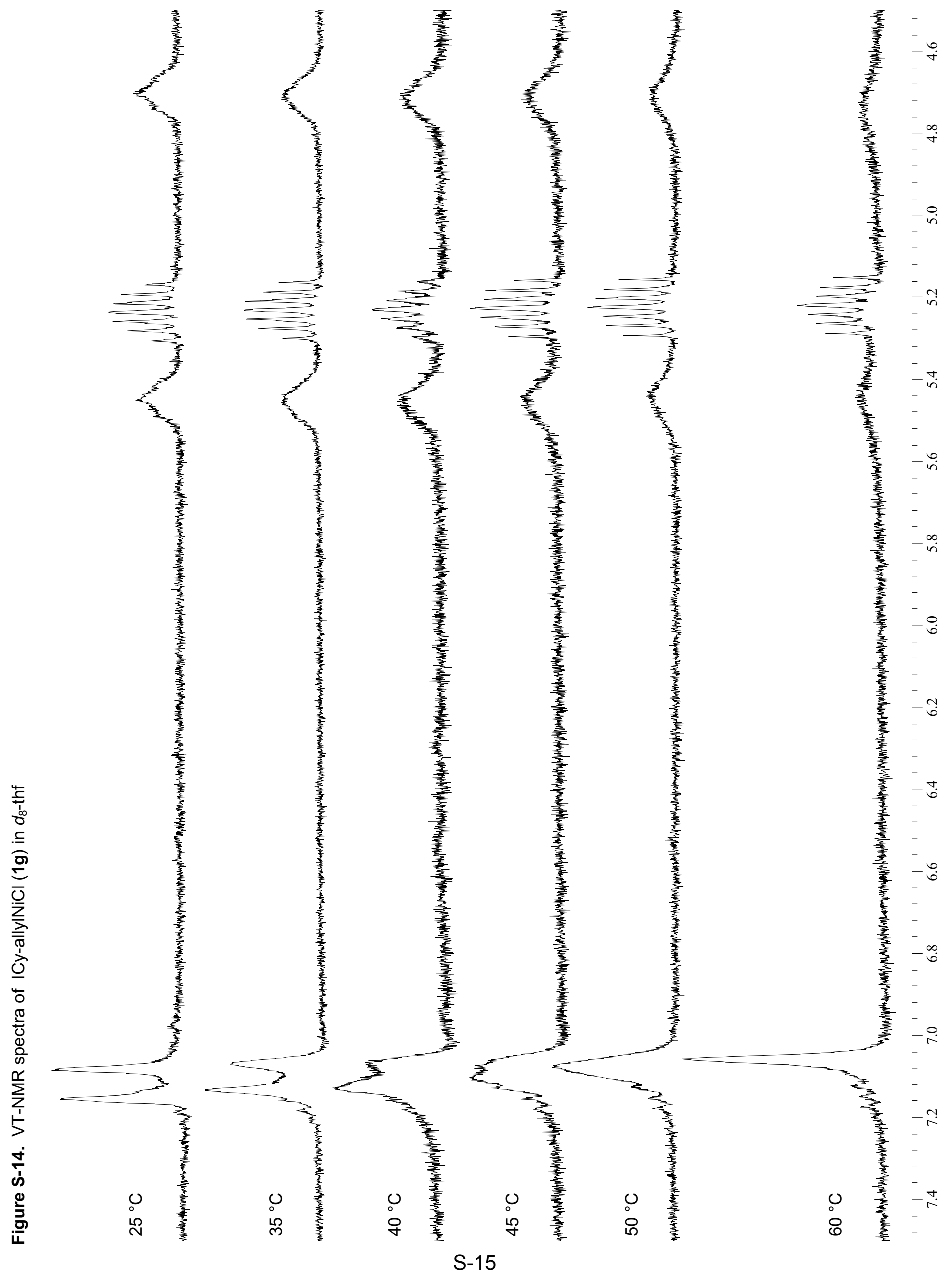




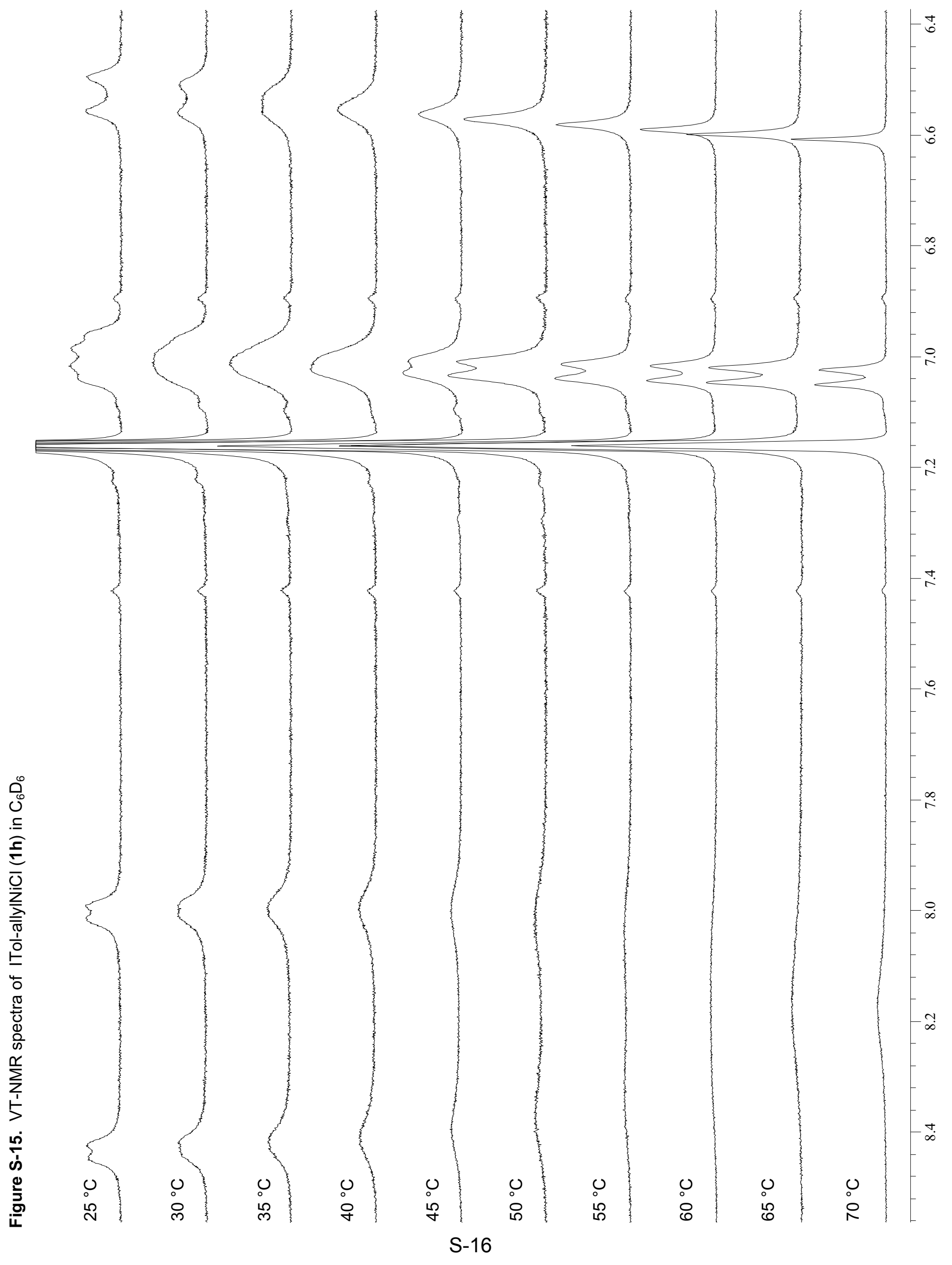




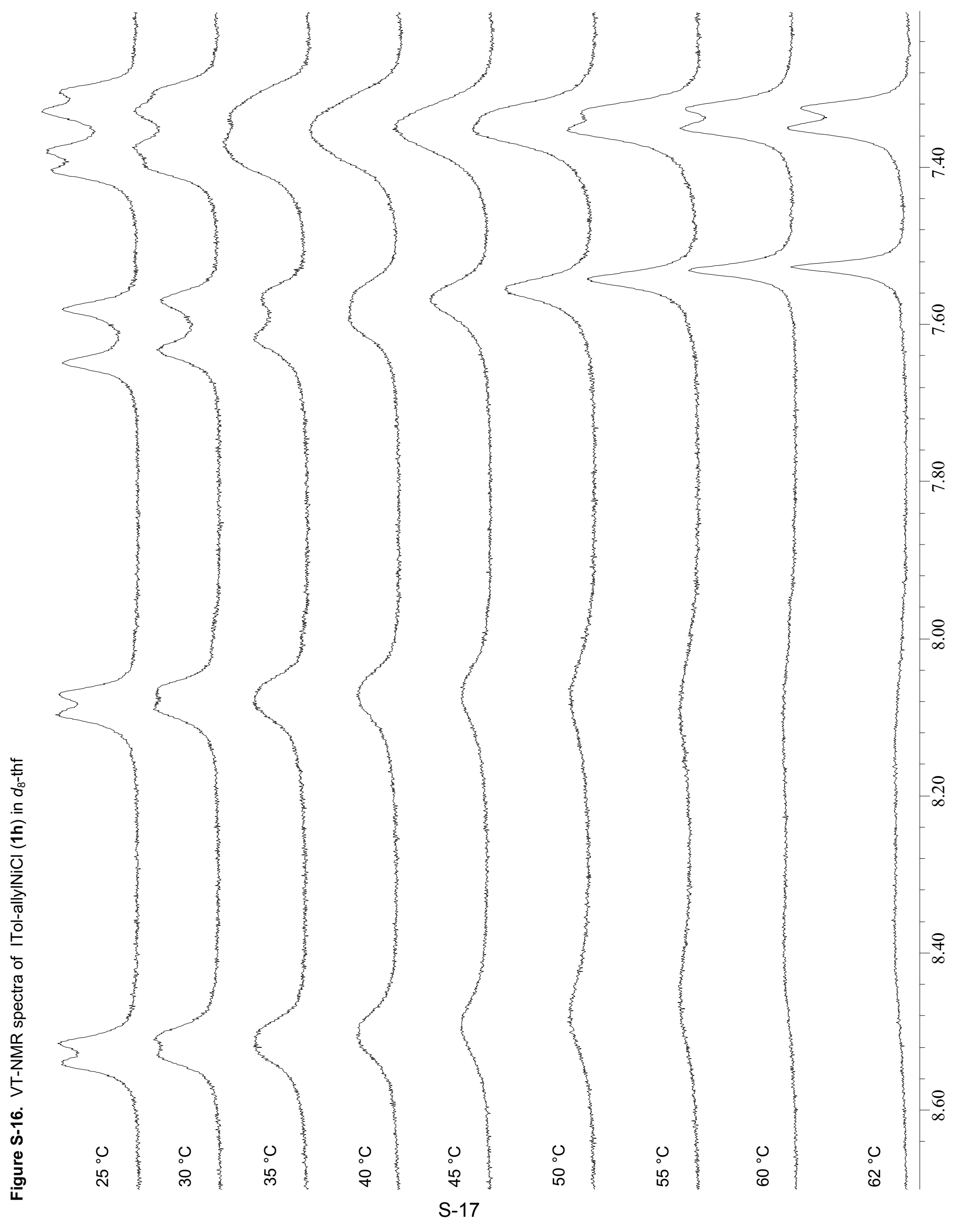


Figure S-17. ORTEP of ItBu-allyINiCl (1d)

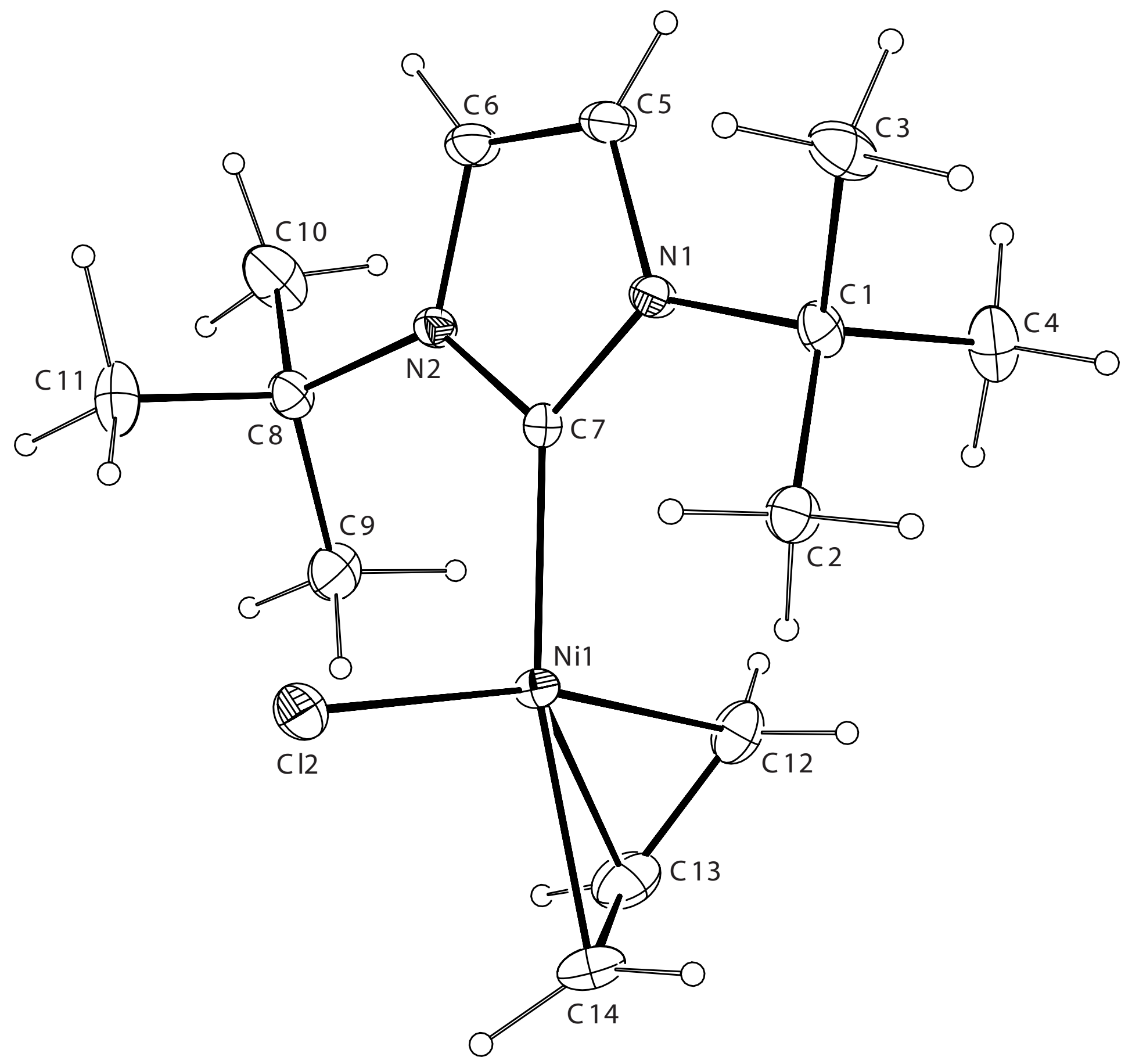


Figure S-18. ORTEP of IBioxMe $\mathrm{O}_{4}$-allyINiCl (1f)

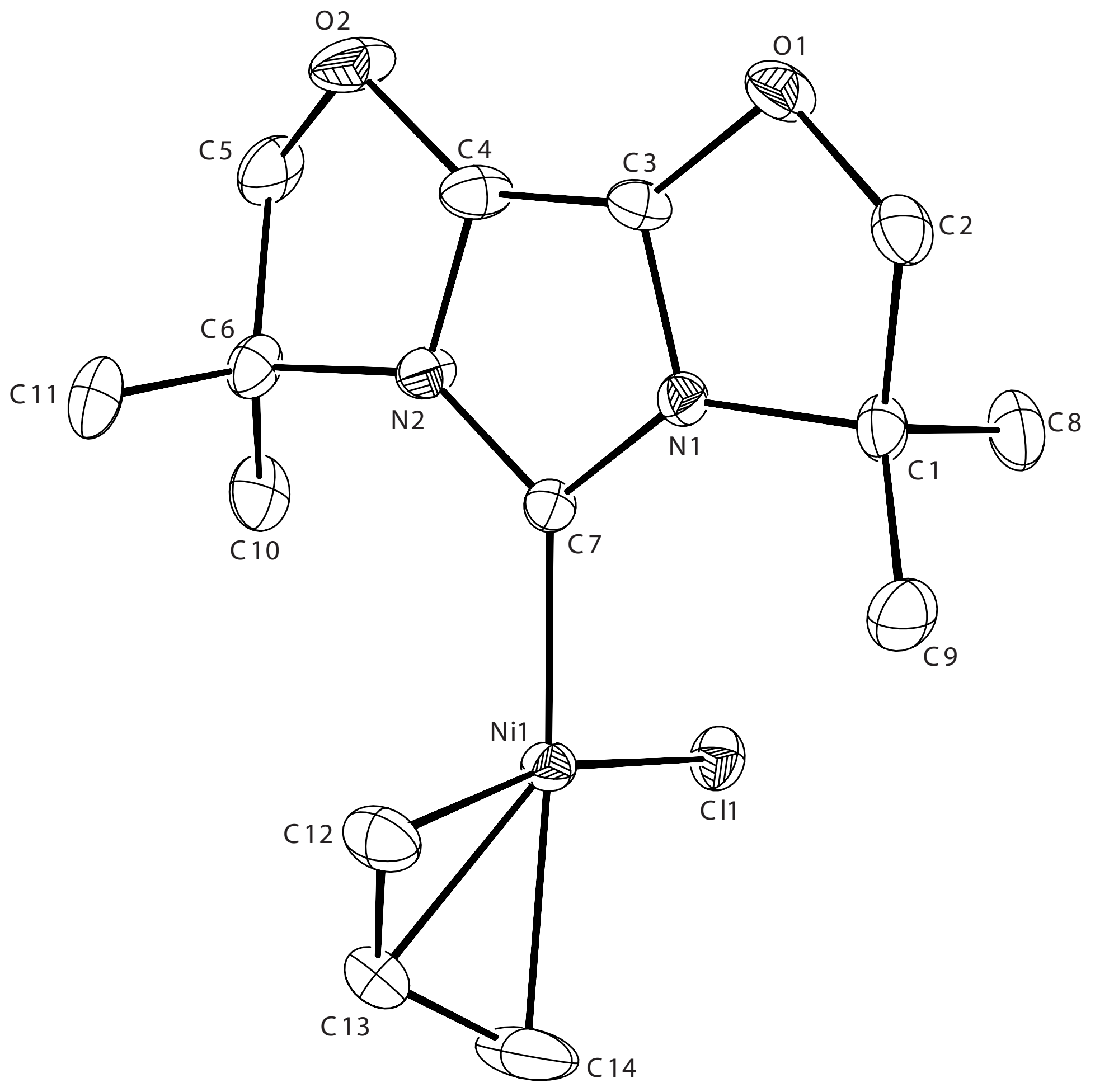


Figure S-19. ORTEP of MeltBu-allyINil (1I)

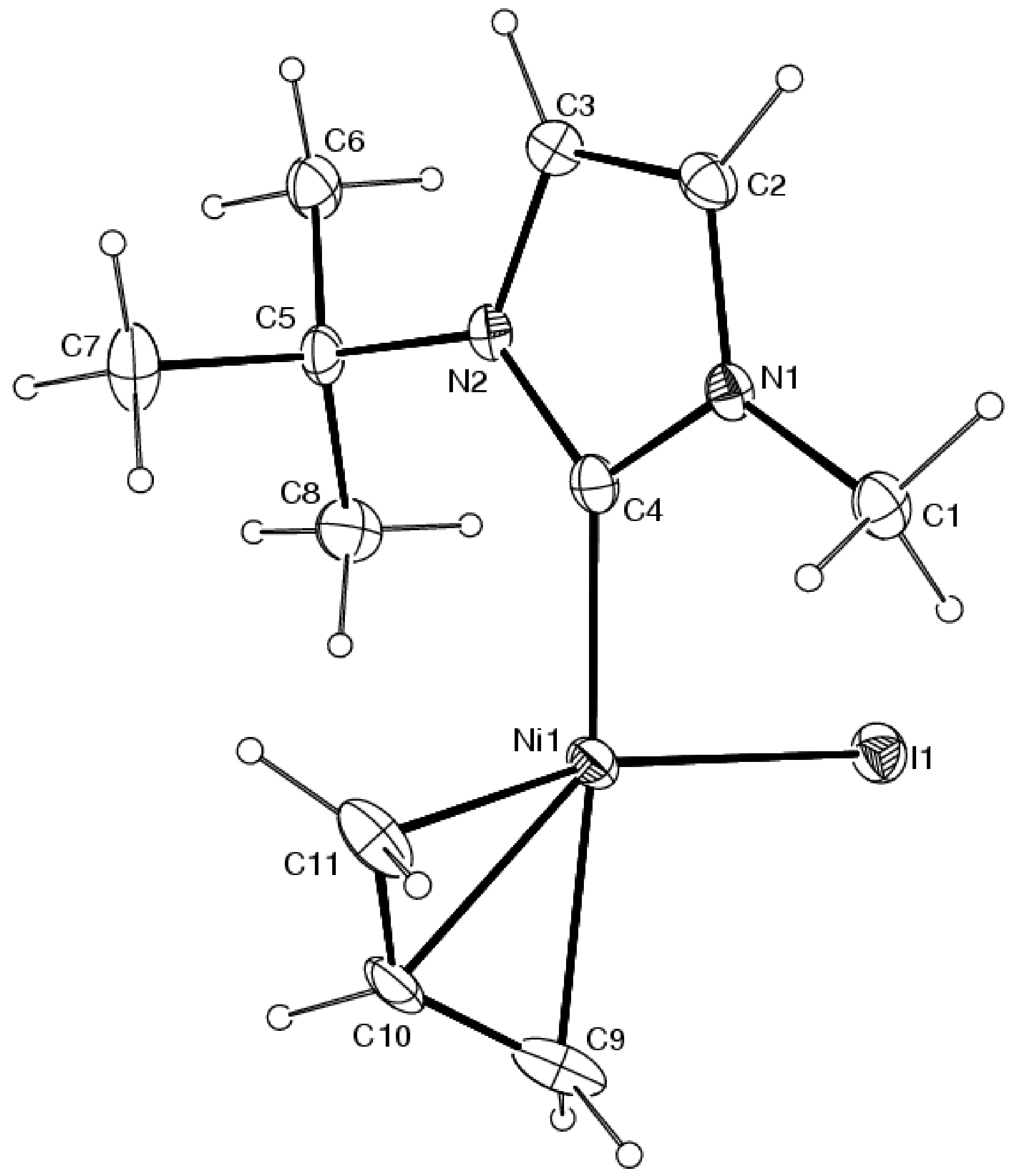


Figure S-20. ORTEP of trans-[ITol $\left.{ }_{2} \mathrm{NiCl}_{2}\right](3 \mathbf{h})$

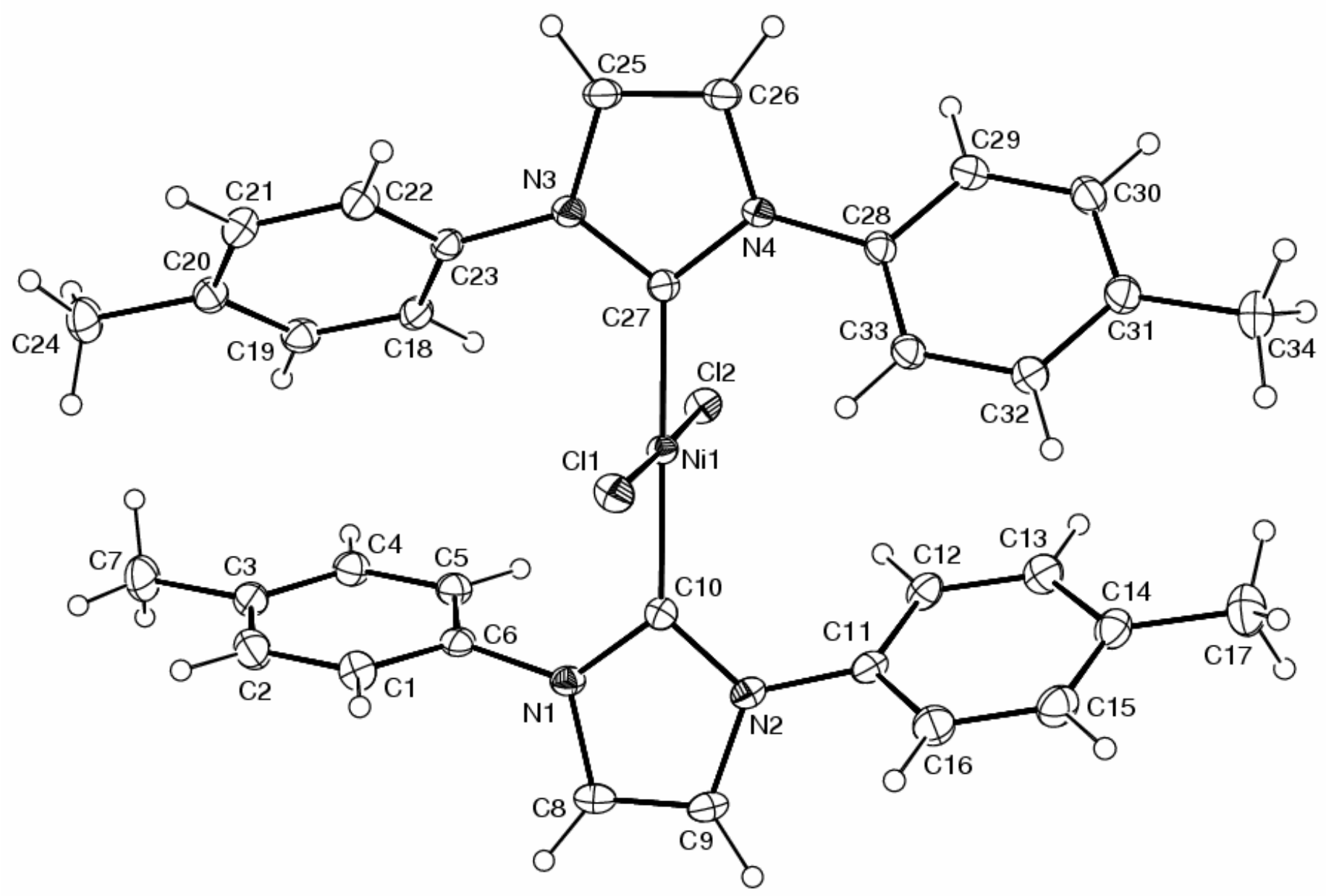




\section{Crystallographic Details.}

allyl(ItBu)NiCl (1d). An orange prism shaped crystal $0.30 \times 0.28 \times 0.25 \mathrm{~mm}$ in size was mounted on a glass fiber with traces of viscous oil and then transferred to a Nonius KappaCCD diffractometer equipped with Mo K $\alpha$ radiation $(\lambda=0.71073 \AA)$. Ten frames of data were collected at $150(1) \mathrm{K}$ with an oscillation range of 1 $\mathrm{deg} /$ frame and an exposure time of $20 \mathrm{sec} /$ frame. ${ }^{1}$ Indexing and unit cell refinement based on all observed reflection from those ten frames, indicated a orthorhombic $P$ lattice. A total of 3259 reflections $\left(\Theta_{\max }=27.47^{\circ}\right)$ were indexed, integrated and corrected for Lorentz, polarization and absorption effects using DENZO-SMN and SCALEPAC. ${ }^{2}$ Post refinement of the unit cell gave $a=10.9968(3) \AA, b=9.6424(3) \AA, c=14.3616(2) \AA$, and $\mathrm{V}=1522.84(7) \AA^{3}$. Axial photographs and systematic absences were consistent with the compound having crystallized in the orthorhombic space group Pna2 ${ }_{1}$.

The structure was solved by a combination of direct methods and heavy atom using SIR $97 .^{3}$ All of the nonhydrogen atoms were refined with anisotropic displacement coefficients. Hydrogen atoms were located and refined isotropically using SHELXL97. ${ }^{4}$ The weighting scheme employed was $\mathrm{w}=1 /\left[\sigma^{2}\left(\mathrm{~F}_{\mathrm{o}}^{2}\right)+(0.0174 \mathrm{P})^{2}+\right.$ $0.6453 \mathrm{P}]$ where $\mathrm{P}=\left(\mathrm{F}_{\mathrm{o}}^{2}+2 \mathrm{~F}_{\mathrm{c}}{ }^{2}\right) / 3$. The refinement converged to $\mathrm{R} 1=0.0240, \mathrm{wR} 2=0.0521$, and $\mathrm{S}=1.054$ for 3048 reflections with $1>2 \sigma(\mathrm{I})$, and $\mathrm{R} 1=0.0279, \mathrm{wR} 2=0.0539$, and $\mathrm{S}=1.054$ for 3259 unique reflections and 265 parameters. ${ }^{5}$ The maximum $\Delta / \sigma$ in the final cycle of the least-squares was 0 , and the residual peaks on the final difference-Fourier map ranged from -0.292 to $0.34 \mathrm{e} / \AA^{3}$. Scattering factors were taken from the International Tables for Crystallography, Volume C. ${ }^{6,7}$

allyl(IBioxMe 4 )Cl (1f). An orange prism shaped crystal $0.35 \times 0.28 \times 0.13 \mathrm{~mm}$ in size was mounted on a glass fiber with traces of viscous oil and then transferred to a Nonius KappaCCD diffractometer equipped with Mo $\mathrm{K} \alpha$ radiation $(\lambda=0.71073 \AA)$. Ten frames of data were collected at $150(1) \mathrm{K}$ with an oscillation range of 1 deg/frame and an exposure time of $20 \mathrm{sec} /$ frame. ${ }^{1}$ Indexing and unit cell refinement based on all observed reflection from those ten frames, indicated a monoclinic $\boldsymbol{P}$ lattice. A total of 7412 reflections $\left(\Theta_{\max }=27.46^{\circ}\right)$ were indexed, integrated and corrected for Lorentz, polarization and absorption effects using DENZO-SMN and

SCALEPAC. ${ }^{2}$ Post refinement of the unit cell gave $a=12.5757(4) \AA, b=10.2031(2) \AA, c=15.6864(4) \AA, \beta$ 
$=113.6613(10)$, and $\mathrm{V}=1843.54(8) \AA^{3}$. Axial photographs and systematic absences were consistent with the compound having crystallized in the monoclinic space group $\boldsymbol{P} 2_{1} / c$.

The structure was solved by a combination of direct methods and heavy atom using SIR 97. ${ }^{3}$ All of the nonhydrogen atoms were refined with anisotropic displacement coefficients. Hydrogen atoms were assigned isotropic displacement coefficients $\mathrm{U}(\mathrm{H})=1.2 \mathrm{U}(\mathrm{C})$ or $1.5 \mathrm{U}(\mathrm{Cmethyl})$, and their coordinates were allowed to ride on their respective carbons using SHELXL97. ${ }^{4}$ The allyl (C13) group and an oxazoline (C5) exhibit orientation disorder (50:50). There is also a disordered toluene solvent molecule sitting on an inversion center in the asymmetric unit. The weighting scheme employed was $\mathrm{w}=1 /\left[\sigma^{2}\left(\mathrm{~F}_{\mathrm{o}}^{2}\right)+(0.0477 \mathrm{P})^{2}+1.4349 \mathrm{P}\right]$ where $\mathrm{P}$ $=\left(\mathrm{F}_{\mathrm{o}}^{2}+2 \mathrm{~F}_{\mathrm{c}}^{2}\right) / 3$. The refinement converged to $\mathrm{R} 1=0.0398, \mathrm{wR} 2=0.0971$, and $\mathrm{S}=1.051$ for 3414 reflections

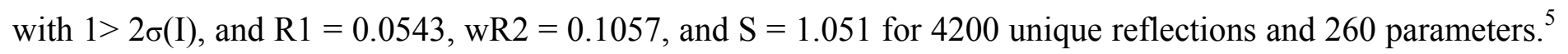
The maximum $\Delta / \sigma$ in the final cycle of the least-squares was 0 , and the residual peaks on the final differenceFourier map ranged from -0.717 to $0.905 \mathrm{e} / \AA^{3}$. Scattering factors were taken from the International Tables for Crystallography, Volume C.,

allyl(MeItBu)NiI (11). An orange plate shaped crystal $0.28 \times 0.20 \times 0.05 \mathrm{~mm}$ in size was mounted on a glass fiber with traces of viscous oil and then transferred to a Nonius KappaCCD diffractometer equipped with Mo $\mathrm{K}^{*}$ radiation $(*=0.71073 \AA)$. Ten frames of data were collected at $150(1) \mathrm{K}$ with an oscillation range of 1 $\mathrm{deg} /$ frame and an exposure time of $20 \mathrm{sec} /$ frame..$^{1}$ Indexing and unit cell refinement based on all observed reflection from those ten frames, indicated a monoclinic $\boldsymbol{P}$ lattice. A total of 5270 reflections $\left(\Theta_{\max }=27.5 *\right)$ were indexed, integrated and corrected for Lorentz, polarization and absorption effects using DENZO-SMN and SCALEPAC. $^{2}$ Post refinement of the unit cell gave $a=8.9469(3) \AA, b=11.6836(3) \AA, c=13.5112(4) \AA, \beta$ $=107.2586(13)$, and $\mathrm{V}=1348.76(7) \AA^{3}$. Axial photographs and systematic absences were consistent with the compound having crystallized in the monoclinic space group $\boldsymbol{P} 2_{1} / c$.

The structure was solved by a combination of direct methods and heavy atom using SIR $97 .^{3}$

All of the non-hydrogen atoms were refined with anisotropic displacement coefficients. Hydrogen atoms were assigned isotropic displacement coefficients $\mathrm{U}(\mathrm{H})=1.2 \mathrm{U}(\mathrm{C})$ or $1.5 \mathrm{U}(\mathrm{Cmethyl})$, and their coordinates were allowed to ride on their respective carbons using SHELXL97. ${ }^{4}$ The allyl group is disordered between two S-23 
orientations with a $65: 35$ ratio. The weighting scheme employed was $\mathrm{w}=1 /\left[\sigma^{2}\left(\mathrm{~F}_{\mathrm{o}}{ }^{2}\right)+(0.0477 \mathrm{P})^{2}+1.4349 \mathrm{P}\right]$ where $\mathrm{P}=\left(\mathrm{F}_{\mathrm{o}}^{2}+2 \mathrm{~F}_{\mathrm{c}}^{2}\right) / 3$. The refinement converged to $\mathrm{R} 1=0.0375$, wR2 $=0.0957$, and $\mathrm{S}=1.058$ for 2619 reflections with $1>2 *(\mathrm{I})$, and $\mathrm{R} 1=0.0467, \mathrm{wR} 2=0.1017$, and $\mathrm{S}=1.058$ for 3074 unique reflections and 150 parameters. ${ }^{5}$ The maximum $\Delta / \sigma$ in the final cycle of the least-squares was 0.001 , and the residual peaks on the final difference-Fourier map ranged from -1.465 to $1.196 \mathrm{e} / \AA^{3}$. Scattering factors were taken from the International Tables for Crystallography, Volume C. ${ }^{6,7}$

trans-(ITol)2NiCl2 (3h). A red-purple plate shaped crystal 0.33 x $0.18 \times 0.13 \mathrm{~mm}$ in size was mounted on a glass fiber with traces of viscous oil and then transferred to a Nonius KappaCCD diffractometer equipped with Mo $\mathrm{K}^{*}$ radiation $(*=0.71073 \AA)$. Ten frames of data were collected at $150(1) \mathrm{K}$ with an oscillation range of 1 deg/frame and an exposure time of $20 \mathrm{sec} /$ frame. ${ }^{1}$ Indexing and unit cell refinement based on all observed reflection from those ten frames, indicated a triclinic $\boldsymbol{P}$ lattice. A total of 10438 reflections $\left(\Theta_{\max }=27.48^{\circ}\right)$ were indexed, integrated and corrected for Lorentz, polarization and absorption effects using DENZO-SMN and SCALEPAC. ${ }^{2}$ Post refinement of the unit cell gave $\mathrm{a}=10.0381(2) \AA, \mathrm{b}=12.4276(3) \AA, \mathrm{c}=13.2040(5) \AA, a=$ 91.5563(12), $\mathrm{b}=111.4776(16), \mathrm{c}=102.8770(16)$, and $\mathrm{V}=1483.48(7) \AA^{3}$. Axial photographs and no systematic absences were consistent with the compound having crystallized in the triclinic space group $\boldsymbol{P}$.

The structure was solved by a combination of direct methods and heavy atom using SIR $97 .^{3}$

All of the non-hydrogen atoms were refined with anisotropic displacement coefficients. Hydrogen atoms were assigned isotropic displacement coefficients $\mathrm{U}(\mathrm{H})=1.2 \mathrm{U}(\mathrm{C})$ or $1.5 \mathrm{U}(\mathrm{Cmethyl})$, and their coordinates were allowed to ride on their respective carbons using SHELXL97. ${ }^{4}$ The weighting scheme employed was $\mathrm{W}=1 /\left[\sigma^{2}\left(\mathrm{~F}_{\mathrm{o}}{ }^{2}\right)+(0.0392 \mathrm{P})^{2}+1.8321 \mathrm{P}\right]$ where $\mathrm{P}=\left(\mathrm{F}_{\mathrm{o}}{ }^{2}+2 \mathrm{~F}_{\mathrm{c}}{ }^{2}\right) / 3$. The refinement converged to $\mathrm{R} 1=0.0449$, $\mathrm{wR} 2=0.1028$, and $\mathrm{S}=1.027$ for 5025 reflections with $1>2 *(\mathrm{I})$, and $\mathrm{R} 1=0.0685, \mathrm{wR} 2=0.1201$, and $\mathrm{S}=1.027$ for 6647 unique reflections and 375 parameters. ${ }^{5}$ The maximum $\Delta / \sigma$ in the final cycle of the least-squares was 0.001, and the residual peaks on the final difference-Fourier map ranged from -0.774 to 0.509 e $/ \AA^{3}$. Scattering factors were taken from the International Tables for Crystallography, Volume C.

ORTEP- $3^{8}$ and WinGX ${ }^{9}$ were used for crystal structure analysis. 


\section{References}

1) COLLECT Data Collection Software. Nonius B.V. 1998.

2) Otwinowski, Z.; Minor, W., "Processing of X-ray Diffraction Data Collected in Oscillation Mode", Methods Enzymol. 1997, 276, 307-326.

3) SIR97 (Release 1.02) - A program for automatic solution and refinement of crystal structure. A. Altomare, M.C. Burla, M. Camalli, G. Cascarano, C. Giacovazzo, A. Guagliardi, A.G. G. Moliteni, G. Polidori, and R. Spagna.

4) SHELX97 [Includes SHELXS97, SHELXL97, CIFTAB ] - Sheldrick, G. M. (1997). Programs for Crystal Structure Analysis (Release 97-2). University of Göttingen, Germany.

5) $\mathrm{R} 1=\Sigma\left(|| \mathrm{F}_{\mathrm{o}}|-| \mathrm{F}_{\mathrm{c}} \|\right) / \Sigma\left|\mathrm{F}_{\mathrm{o}}\right|$, wR2 $=\left[\Sigma\left(\mathrm{w}\left(\mathrm{F}_{\mathrm{o}}{ }^{2}-\mathrm{F}_{\mathrm{c}}{ }^{2}\right) 2\right) / \Sigma\left(\mathrm{F}_{\mathrm{o}}{ }^{2}\right)^{2}\right]^{1 / 2}$, and $\mathrm{S}=$ Goodness-of-fit on $\mathrm{F}^{2}=[\Sigma($ $\left.\mathrm{w}\left(\mathrm{F}_{\mathrm{o}}{ }^{2}-\mathrm{F}_{\mathrm{c}}{ }^{2}\right)^{2} /(\mathrm{n}-\mathrm{p})\right]^{1 / 2}$, where $\mathrm{n}$ is the number of reflections and $\mathrm{p}$ is the number of parameters refined.

6) Maslen, E. N.; Fox, A. G.; O’Keefe, M. A., International Tables for Crystallography: Mathemetical, Physical and Chemical Tables, Vol. C, Chapter 6, Wilson, A. J. C., Ed.; Kluwer, Dordrecht, The Netherlands, 1992; pp. 476-516.

7) Creagh, D. C.; McdAuley, W. J., International Tables for Crystallography: mathematical, Physical and Chemical tables, Vol. C, Chapter 4 Wilson, A. J. C., Ed.; Kluwer, Dordrecht, The Netherlands, $1992 ;$ pp. 206-222.

8) ORTEP3 for Windows - L. J. Farrugia, J. Appl. Crystallogr. 1997, 30, 565.

9) WinGX A Windows Program for Crystal Structure Analysis. L. J. Farrugia, University of Glasgow, Glasgow, 1998. 
Table S-1. Crystal data and structure refinement for allyl(ItBu) $\mathrm{NiCl}$ (1d).

Identification code

Empirical formula

Formula weight

Temperature

Wavelength

Crystal system

Space group

Unit cell dimensions

Volume

Z

Density (calculated)

Absorption coefficient

$\mathrm{F}(000)$

Crystal size

Theta range for data collection

Index ranges

Reflections collected

Independent reflections

Completeness to theta $=27.47^{\circ}$

Absorption correction

Max. and min. transmission

Refinement method

Data / restraints / parameters

Goodness-of-fit on F2

Final $\mathrm{R}$ indices [I $>2 \operatorname{sigma}(\mathrm{I})]$

$\mathrm{R}$ indices (all data)

Absolute structure parameter

Extinction coefficient

Largest diff. peak and hole mss022

$\mathrm{C}_{14} \mathrm{H}_{25} \mathrm{ClN}_{2} \mathrm{Ni}$

315.52

150(1) K

$0.71073 \AA$

Orthorhombic

Pna2 1

$$
\begin{array}{ll}
\mathrm{a}=10.9968(3) \AA & \alpha=90^{\circ} . \\
\mathrm{b}=9.6424(3) \AA & \beta=90^{\circ} . \\
\mathrm{c}=14.3616(2) \AA & \gamma=90^{\circ} .
\end{array}
$$

1522.84(7) $\AA 3$

4

$1.376 \mathrm{Mg} / \mathrm{m}^{3}$

$1.435 \mathrm{~mm}^{-1}$

672

$0.30 \times 0.28 \times 0.25 \mathrm{~mm}^{3}$

2.81 to $27.47^{\circ}$.

$-14<=\mathrm{h}<=14,-12<=\mathrm{k}<=12,-18<=\mathrm{l}<=18$

3259

$3259[\mathrm{R}(\mathrm{int})=0.0000]$

$99.4 \%$

Multi-scan

0.7155 and 0.6727

Full-matrix least-squares on $\mathrm{F}^{2}$

3259 / 1 / 265

1.054

$\mathrm{R} 1=0.0240, \mathrm{wR} 2=0.0521$

$\mathrm{R} 1=0.0279, \mathrm{wR} 2=0.0539$

$0.519(13)$

$0.0032(8)$

0.340 and -0.292 e. $\AA^{-3}$ 
Table S-2. Atomic coordinates ( x $\left.10^{4}\right)$ and equivalent isotropic displacement parameters $\left(\AA^{2} \times 10^{3}\right)$ for allyl $(\mathrm{ItBu}) \mathrm{NiCl}(\mathbf{1 d})$. $\mathrm{U}(\mathrm{eq})$ is defined as one third of the trace of the orthogonalized $\mathrm{U}^{\mathrm{ij}}$ tensor.

\begin{tabular}{lrrrr}
\hline & $\mathrm{X}$ & $\mathrm{y}$ & $\mathrm{z}$ & $\mathrm{U}(\mathrm{eq})$ \\
\hline $\mathrm{Ni}(1)$ & $2459(1)$ & $-1025(1)$ & $-1270(1)$ & $18(1)$ \\
$\mathrm{Cl}(2)$ & $998(1)$ & $-990(1)$ & $-2349(1)$ & $27(1)$ \\
$\mathrm{N}(1)$ & $2153(2)$ & $1840(2)$ & $-533(1)$ & $19(1)$ \\
$\mathrm{N}(2)$ & $3388(2)$ & $1785(2)$ & $-1706(1)$ & $18(1)$ \\
$\mathrm{C}(1)$ & $1354(2)$ & $1477(2)$ & $283(1)$ & $23(1)$ \\
$\mathrm{C}(2)$ & $624(2)$ & $167(2)$ & $80(2)$ & $25(1)$ \\
$\mathrm{C}(3)$ & $448(3)$ & $2677(3)$ & $438(2)$ & $33(1)$ \\
$\mathrm{C}(4)$ & $2147(3)$ & $1300(4)$ & $1139(2)$ & $36(1)$ \\
$\mathrm{C}(5)$ & $2525(2)$ & $3195(2)$ & $-704(2)$ & $26(1)$ \\
$\mathrm{C}(6)$ & $3274(2)$ & $3169(2)$ & $-1436(2)$ & $25(1)$ \\
$\mathrm{C}(7)$ & $2683(2)$ & $952(2)$ & $-1154(2)$ & $16(1)$ \\
$\mathrm{C}(8)$ & $4103(2)$ & $1367(2)$ & $-2548(1)$ & $23(1)$ \\
$\mathrm{C}(9)$ & $4618(2)$ & $-80(2)$ & $-2425(2)$ & $34(1)$ \\
$\mathrm{C}(10)$ & $5161(3)$ & $2369(3)$ & $-2679(2)$ & $44(1)$ \\
$\mathrm{C}(11)$ & $3268(2)$ & $1413(3)$ & $-3388(2)$ & $35(1)$ \\
$\mathrm{C}(12)$ & $3665(2)$ & $-1543(3)$ & $-290(2)$ & $33(1)$ \\
$\mathrm{C}(13)$ & $3499(3)$ & $-2623(3)$ & $-927(2)$ & $39(1)$ \\
$\mathrm{C}(14)$ & $2354(3)$ & $-3138(3)$ & $-1097(2)$ & $38(1)$ \\
& & & & \\
\hline & & & & \\
\hline
\end{tabular}


Table S-3. Bond lengths $[\AA]$ and angles $\left[{ }^{\circ}\right]$ for allyl $(\mathrm{ItBu}) \mathrm{NiCl}(\mathbf{1 d})$.

\begin{tabular}{ll}
\hline $\mathrm{Ni}(1)-\mathrm{C}(7)$ & $1.9290(18)$ \\
$\mathrm{Ni}(1)-\mathrm{C}(13)$ & $1.982(2)$ \\
$\mathrm{Ni}(1)-\mathrm{C}(12)$ & $1.998(2)$ \\
$\mathrm{Ni}(1)-\mathrm{C}(14)$ & $2.056(2)$ \\
$\mathrm{Ni}(1)-\mathrm{Cl}(2)$ & $2.2319(7)$ \\
$\mathrm{N}(1)-\mathrm{C}(7)$ & $1.367(3)$ \\
$\mathrm{N}(1)-\mathrm{C}(5)$ & $1.391(3)$ \\
$\mathrm{N}(1)-\mathrm{C}(1)$ & $1.506(2)$ \\
$\mathrm{N}(2)-\mathrm{C}(7)$ & $1.369(3)$ \\
$\mathrm{N}(2)-\mathrm{C}(6)$ & $1.396(2)$ \\
$\mathrm{N}(2)-\mathrm{C}(8)$ & $1.498(3)$ \\
$\mathrm{C}(1)-\mathrm{C}(4)$ & $1.517(3)$ \\
$\mathrm{C}(1)-\mathrm{C}(2)$ & $1.525(3)$ \\
$\mathrm{C}(1)-\mathrm{C}(3)$ & $1.543(3)$ \\
$\mathrm{C}(2)-\mathrm{H}(2 \mathrm{~A})$ & $1.00(3)$ \\
$\mathrm{C}(2)-\mathrm{H}(2 \mathrm{~B})$ & $0.94(2)$ \\
$\mathrm{C}(2)-\mathrm{H}(2 \mathrm{C})$ & $1.05(3)$ \\
$\mathrm{C}(3)-\mathrm{H}(3 \mathrm{~A})$ & $1.00(3)$ \\
$\mathrm{C}(3)-\mathrm{H}(3 \mathrm{~B})$ & $0.97(3)$ \\
$\mathrm{C}(3)-\mathrm{H}(3 \mathrm{C})$ & $0.94(3)$ \\
$\mathrm{C}(4)-\mathrm{H}(4 \mathrm{~A})$ & $0.92(3)$ \\
$\mathrm{C}(4)-\mathrm{H}(4 \mathrm{~B})$ & $1.03(3)$ \\
$\mathrm{C}(4)-\mathrm{H}(4 \mathrm{C})$ & $0.96(3)$ \\
$\mathrm{C}(5)-\mathrm{C}(6)$ & $1.335(3)$ \\
$\mathrm{C}(5)-\mathrm{H}(5)$ & $0.86(3)$ \\
$\mathrm{C}(6)-\mathrm{H}(6)$ & $0.94(2)$ \\
$\mathrm{C}(8)-\mathrm{C}(9)$ & $1.516(3)$ \\
$\mathrm{C}(8)-\mathrm{C}(11)$ & $1.516(3)$ \\
$\mathrm{C}(8)-\mathrm{C}(10)$ & $1.524(3)$ \\
$\mathrm{C}(9)-\mathrm{H}(9 \mathrm{~A})$ & $0.96(3)$ \\
$\mathrm{C}(9)-\mathrm{H}(9 \mathrm{~B})$ & $1.00(2)$ \\
$\mathrm{C}(9)-\mathrm{H}(9 \mathrm{C})$ & $1.04(3)$ \\
$\mathrm{C}(10)-\mathrm{H}(10 \mathrm{~A})$ & $0.97(3)$ \\
$\mathrm{C}(10)-\mathrm{H}(10 \mathrm{~B})$ & $0.92(4)$ \\
$\mathrm{C}(10)-\mathrm{H}(10 \mathrm{C})$ & $1.01(4)$ \\
$\mathrm{C}(11)-\mathrm{H}(11 \mathrm{~A})$ & $0.99(3)$ \\
$\mathrm{C}(11)-\mathrm{H}(11 \mathrm{~B})$ &
\end{tabular}




\begin{tabular}{|c|c|}
\hline $\mathrm{C}(11)-\mathrm{H}(11 \mathrm{C})$ & $1.11(4)$ \\
\hline$C(12)-C(13)$ & $1.397(4)$ \\
\hline $\mathrm{C}(12)-\mathrm{H}(12 \mathrm{~A})$ & $0.96(3)$ \\
\hline $\mathrm{C}(12)-\mathrm{H}(12 \mathrm{~B})$ & $0.95(3)$ \\
\hline$C(13)-C(14)$ & $1.376(4)$ \\
\hline $\mathrm{C}(13)-\mathrm{H}(13)$ & $1.10(3)$ \\
\hline $\mathrm{C}(14)-\mathrm{H}(14 \mathrm{~A})$ & $1.06(3)$ \\
\hline $\mathrm{C}(14)-\mathrm{H}(14 \mathrm{~B})$ & $0.97(4)$ \\
\hline $\mathrm{C}(7)-\mathrm{Ni}(1)-\mathrm{C}(13)$ & $132.28(10)$ \\
\hline $\mathrm{C}(7)-\mathrm{Ni}(1)-\mathrm{C}(12)$ & $95.81(10)$ \\
\hline $\mathrm{C}(13)-\mathrm{Ni}(1)-\mathrm{C}(12)$ & $41.10(11)$ \\
\hline $\mathrm{C}(7)-\mathrm{Ni}(1)-\mathrm{C}(14)$ & $167.37(11)$ \\
\hline$C(13)-N i(1)-C(14)$ & $39.78(11)$ \\
\hline $\mathrm{C}(12)-\mathrm{Ni}(1)-\mathrm{C}(14)$ & $72.76(12)$ \\
\hline $\mathrm{C}(7)-\mathrm{Ni}(1)-\mathrm{Cl}(2)$ & $97.91(6)$ \\
\hline $\mathrm{C}(13)-\mathrm{Ni}(1)-\mathrm{Cl}(2)$ & $126.88(8)$ \\
\hline $\mathrm{C}(12)-\mathrm{Ni}(1)-\mathrm{Cl}(2)$ & $166.09(8)$ \\
\hline $\mathrm{C}(14)-\mathrm{Ni}(1)-\mathrm{Cl}(2)$ & $93.36(9)$ \\
\hline $\mathrm{C}(7)-\mathrm{N}(1)-\mathrm{C}(5)$ & $110.36(17)$ \\
\hline $\mathrm{C}(7)-\mathrm{N}(1)-\mathrm{C}(1)$ & $127.63(17)$ \\
\hline $\mathrm{C}(5)-\mathrm{N}(1)-\mathrm{C}(1)$ & $121.84(17)$ \\
\hline $\mathrm{C}(7)-\mathrm{N}(2)-\mathrm{C}(6)$ & $110.44(18)$ \\
\hline $\mathrm{C}(7)-\mathrm{N}(2)-\mathrm{C}(8)$ & $127.39(17)$ \\
\hline $\mathrm{C}(6)-\mathrm{N}(2)-\mathrm{C}(8)$ & $121.90(17)$ \\
\hline$N(1)-C(1)-C(4)$ & $108.77(18)$ \\
\hline $\mathrm{N}(1)-\mathrm{C}(1)-\mathrm{C}(2)$ & $110.53(16)$ \\
\hline $\mathrm{C}(4)-\mathrm{C}(1)-\mathrm{C}(2)$ & $111.4(2)$ \\
\hline $\mathrm{N}(1)-\mathrm{C}(1)-\mathrm{C}(3)$ & $108.34(18)$ \\
\hline $\mathrm{C}(4)-\mathrm{C}(1)-\mathrm{C}(3)$ & $109.8(2)$ \\
\hline $\mathrm{C}(2)-\mathrm{C}(1)-\mathrm{C}(3)$ & $108.01(19)$ \\
\hline $\mathrm{C}(1)-\mathrm{C}(2)-\mathrm{H}(2 \mathrm{~A})$ & $111.0(16)$ \\
\hline $\mathrm{C}(1)-\mathrm{C}(2)-\mathrm{H}(2 \mathrm{~B})$ & $110.7(14)$ \\
\hline $\mathrm{H}(2 \mathrm{~A})-\mathrm{C}(2)-\mathrm{H}(2 \mathrm{~B})$ & $107(2)$ \\
\hline $\mathrm{C}(1)-\mathrm{C}(2)-\mathrm{H}(2 \mathrm{C})$ & $108.1(15)$ \\
\hline $\mathrm{H}(2 \mathrm{~A})-\mathrm{C}(2)-\mathrm{H}(2 \mathrm{C})$ & $112(2)$ \\
\hline $\mathrm{H}(2 \mathrm{~B})-\mathrm{C}(2)-\mathrm{H}(2 \mathrm{C})$ & $108(2)$ \\
\hline $\mathrm{C}(1)-\mathrm{C}(3)-\mathrm{H}(3 \mathrm{~A})$ & $106.6(14)$ \\
\hline $\mathrm{C}(1)-\mathrm{C}(3)-\mathrm{H}(3 \mathrm{~B})$ & $113.6(17)$ \\
\hline
\end{tabular}




\begin{tabular}{|c|c|}
\hline $\mathrm{H}(3 \mathrm{~A})-\mathrm{C}(3)-\mathrm{H}(3 \mathrm{~B})$ & $108(2)$ \\
\hline $\mathrm{C}(1)-\mathrm{C}(3)-\mathrm{H}(3 \mathrm{C})$ & $111.5(17)$ \\
\hline $\mathrm{H}(3 \mathrm{~A})-\mathrm{C}(3)-\mathrm{H}(3 \mathrm{C})$ & $101(2)$ \\
\hline $\mathrm{H}(3 \mathrm{~B})-\mathrm{C}(3)-\mathrm{H}(3 \mathrm{C})$ & $115(2)$ \\
\hline $\mathrm{C}(1)-\mathrm{C}(4)-\mathrm{H}(4 \mathrm{~A})$ & $107.3(19)$ \\
\hline $\mathrm{C}(1)-\mathrm{C}(4)-\mathrm{H}(4 \mathrm{~B})$ & $107.6(18)$ \\
\hline $\mathrm{H}(4 \mathrm{~A})-\mathrm{C}(4)-\mathrm{H}(4 \mathrm{~B})$ & $114(2)$ \\
\hline $\mathrm{C}(1)-\mathrm{C}(4)-\mathrm{H}(4 \mathrm{C})$ & $109(2)$ \\
\hline $\mathrm{H}(4 \mathrm{~A})-\mathrm{C}(4)-\mathrm{H}(4 \mathrm{C})$ & $110(3)$ \\
\hline $\mathrm{H}(4 \mathrm{~B})-\mathrm{C}(4)-\mathrm{H}(4 \mathrm{C})$ & $110(3)$ \\
\hline $\mathrm{C}(6)-\mathrm{C}(5)-\mathrm{N}(1)$ & $107.64(19)$ \\
\hline $\mathrm{C}(6)-\mathrm{C}(5)-\mathrm{H}(5)$ & $129.7(17)$ \\
\hline $\mathrm{N}(1)-\mathrm{C}(5)-\mathrm{H}(5)$ & $122.7(18)$ \\
\hline $\mathrm{C}(5)-\mathrm{C}(6)-\mathrm{N}(2)$ & $106.96(18)$ \\
\hline $\mathrm{C}(5)-\mathrm{C}(6)-\mathrm{H}(6)$ & $131.7(14)$ \\
\hline $\mathrm{N}(2)-\mathrm{C}(6)-\mathrm{H}(6)$ & $121.3(14)$ \\
\hline $\mathrm{N}(1)-\mathrm{C}(7)-\mathrm{N}(2)$ & $104.58(15)$ \\
\hline N(1)-C(7)-Ni(1) & $128.42(17)$ \\
\hline $\mathrm{N}(2)-\mathrm{C}(7)-\mathrm{Ni}(1)$ & $126.97(18)$ \\
\hline $\mathrm{N}(2)-\mathrm{C}(8)-\mathrm{C}(9)$ & $110.43(17)$ \\
\hline $\mathrm{N}(2)-\mathrm{C}(8)-\mathrm{C}(11)$ & $108.50(17)$ \\
\hline C(9)-C(8)-C(11) & $110.3(2)$ \\
\hline $\mathrm{N}(2)-\mathrm{C}(8)-\mathrm{C}(10)$ & $109.26(19)$ \\
\hline$C(9)-C(8)-C(10)$ & $108.2(2)$ \\
\hline $\mathrm{C}(11)-\mathrm{C}(8)-\mathrm{C}(10)$ & $110.2(2)$ \\
\hline $\mathrm{C}(8)-\mathrm{C}(9)-\mathrm{H}(9 \mathrm{~A})$ & $110.5(18)$ \\
\hline C(8)-C(9)-H(9B) & $114.8(12)$ \\
\hline $\mathrm{H}(9 \mathrm{~A})-\mathrm{C}(9)-\mathrm{H}(9 \mathrm{~B})$ & $104(2)$ \\
\hline C(8)-C(9)-H(9C) & $110.0(17)$ \\
\hline $\mathrm{H}(9 \mathrm{~A})-\mathrm{C}(9)-\mathrm{H}(9 \mathrm{C})$ & $114(2)$ \\
\hline H(9B)-C(9)-H(9C) & $103(2)$ \\
\hline $\mathrm{C}(8)-\mathrm{C}(10)-\mathrm{H}(10 \mathrm{~A})$ & $111.7(18)$ \\
\hline $\mathrm{C}(8)-\mathrm{C}(10)-\mathrm{H}(10 \mathrm{~B})$ & $110(2)$ \\
\hline $\mathrm{H}(10 \mathrm{~A})-\mathrm{C}(10)-\mathrm{H}(10 \mathrm{~B})$ & $105(3)$ \\
\hline $\mathrm{C}(8)-\mathrm{C}(10)-\mathrm{H}(10 \mathrm{C})$ & $108(2)$ \\
\hline $\mathrm{H}(10 \mathrm{~A})-\mathrm{C}(10)-\mathrm{H}(10 \mathrm{C})$ & 113(3) \\
\hline $\mathrm{H}(10 \mathrm{~B})-\mathrm{C}(10)-\mathrm{H}(10 \mathrm{C})$ & $108(3)$ \\
\hline $\mathrm{C}(8)-\mathrm{C}(11)-\mathrm{H}(11 \mathrm{~A})$ & $109.0(17)$ \\
\hline $\mathrm{C}(8)-\mathrm{C}(11)-\mathrm{H}(11 \mathrm{~B})$ & $117(2)$ \\
\hline
\end{tabular}




$\begin{array}{ll}\mathrm{H}(11 \mathrm{~A})-\mathrm{C}(11)-\mathrm{H}(11 \mathrm{~B}) & 104(3) \\ \mathrm{C}(8)-\mathrm{C}(11)-\mathrm{H}(11 \mathrm{C}) & 105.6(19) \\ \mathrm{H}(11 \mathrm{~A})-\mathrm{C}(11)-\mathrm{H}(11 \mathrm{C}) & 107(2) \\ \mathrm{H}(11 \mathrm{~B})-\mathrm{C}(11)-\mathrm{H}(11 \mathrm{C}) & 114(3) \\ \mathrm{C}(13)-\mathrm{C}(12)-\mathrm{Ni}(1) & 68.82(14) \\ \mathrm{C}(13)-\mathrm{C}(12)-\mathrm{H}(12 \mathrm{~A}) & 117.6(18) \\ \mathrm{Ni}(1)-\mathrm{C}(12)-\mathrm{H}(12 \mathrm{~A}) & 104.9(16) \\ \mathrm{C}(13)-\mathrm{C}(12)-\mathrm{H}(12 \mathrm{~B}) & 127.5(16) \\ \mathrm{Ni}(1)-\mathrm{C}(12)-\mathrm{H}(12 \mathrm{~B}) & 125.5(15) \\ \mathrm{H}(12 \mathrm{~A})-\mathrm{C}(12)-\mathrm{H}(12 \mathrm{~B}) & 107(2) \\ \mathrm{C}(14)-\mathrm{C}(13)-\mathrm{C}(12) & 120.3(2) \\ \mathrm{C}(14)-\mathrm{C}(13)-\mathrm{Ni}(1) & 73.01(15) \\ \mathrm{C}(12)-\mathrm{C}(13)-\mathrm{Ni}(1) & 70.08(14) \\ \mathrm{C}(14)-\mathrm{C}(13)-\mathrm{H}(13) & 122.5(16) \\ \mathrm{C}(12)-\mathrm{C}(13)-\mathrm{H}(13) & 114.5(15) \\ \mathrm{Ni}(1)-\mathrm{C}(13)-\mathrm{H}(13) & 113.0(15) \\ \mathrm{C}(13)-\mathrm{C}(14)-\mathrm{Ni}(1) & 67.20(14) \\ \mathrm{C}(13)-\mathrm{C}(14)-\mathrm{H}(14 \mathrm{~A}) & 121.4(15) \\ \mathrm{Ni}(1)-\mathrm{C}(14)-\mathrm{H}(14 \mathrm{~A}) & 97.6(15) \\ \mathrm{C}(13)-\mathrm{C}(14)-\mathrm{H}(14 \mathrm{~B}) & 113(2) \\ \mathrm{Ni}(1)-\mathrm{C}(14)-\mathrm{H}(14 \mathrm{~B}) & 114(2) \\ \mathrm{H}(14 \mathrm{~A})-\mathrm{C}(14)-\mathrm{H}(14 \mathrm{~B}) & 124(3) \\ & \end{array}$

Symmetry transformations used to generate equivalent atoms: 
Table S-4. Anisotropic displacement parameters $\left(\AA^{2} \times 10^{3}\right)$ for allyl(ItBu)NiCl (1d). The anisotropic displacement factor exponent takes the form: $-2 \pi^{2}\left[h^{2} a^{* 2} U^{11}+\ldots+2 h k a^{*} b^{*} U^{12}\right]$

\begin{tabular}{lcccccc}
\hline & $\mathrm{U}^{11}$ & $\mathrm{U}^{22}$ & $\mathrm{U}^{33}$ & $\mathrm{U}^{23}$ & $\mathrm{U}^{13}$ & $\mathrm{U}^{12}$ \\
\hline $\mathrm{Ni}(1)$ & $22(1)$ & $14(1)$ & $19(1)$ & $1(1)$ & $1(1)$ & $0(1)$ \\
$\mathrm{Cl}(2)$ & $27(1)$ & $28(1)$ & $26(1)$ & $-2(1)$ & $-6(1)$ & $-3(1)$ \\
$\mathrm{N}(1)$ & $21(1)$ & $16(1)$ & $20(1)$ & $-3(1)$ & $3(1)$ & $-2(1)$ \\
$\mathrm{N}(2)$ & $21(1)$ & $16(1)$ & $18(1)$ & $-1(1)$ & $1(1)$ & $-3(1)$ \\
$\mathrm{C}(1)$ & $22(1)$ & $28(1)$ & $18(1)$ & $-3(1)$ & $6(1)$ & $0(1)$ \\
$\mathrm{C}(2)$ & $24(1)$ & $26(1)$ & $24(1)$ & $0(1)$ & $5(1)$ & $-1(1)$ \\
$\mathrm{C}(3)$ & $31(1)$ & $29(1)$ & $38(1)$ & $-10(1)$ & $11(1)$ & $1(1)$ \\
$\mathrm{C}(4)$ & $33(1)$ & $52(2)$ & $22(1)$ & $-1(1)$ & $0(1)$ & $-9(1)$ \\
$\mathrm{C}(5)$ & $32(1)$ & $16(1)$ & $29(1)$ & $-3(1)$ & $4(1)$ & $0(1)$ \\
$\mathrm{C}(6)$ & $31(1)$ & $17(1)$ & $29(1)$ & $-1(1)$ & $5(1)$ & $-4(1)$ \\
$\mathrm{C}(7)$ & $16(1)$ & $18(1)$ & $14(1)$ & $1(1)$ & $-1(1)$ & $0(1)$ \\
$\mathrm{C}(8)$ & $22(1)$ & $25(1)$ & $21(1)$ & $-2(1)$ & $4(1)$ & $-3(1)$ \\
$\mathrm{C}(9)$ & $38(1)$ & $35(1)$ & $28(1)$ & $1(1)$ & $13(1)$ & $10(1)$ \\
$\mathrm{C}(10)$ & $45(2)$ & $48(2)$ & $39(2)$ & $-12(1)$ & $20(1)$ & $-23(1)$ \\
$\mathrm{C}(11)$ & $32(1)$ & $56(2)$ & $19(1)$ & $1(1)$ & $1(1)$ & $5(1)$ \\
$\mathrm{C}(12)$ & $32(1)$ & $38(1)$ & $29(1)$ & $9(1)$ & $-7(1)$ & $4(1)$ \\
$\mathrm{C}(13)$ & $41(1)$ & $29(1)$ & $49(2)$ & $9(1)$ & $1(1)$ & $12(1)$ \\
$\mathrm{C}(14)$ & $55(2)$ & $16(1)$ & $43(2)$ & $6(1)$ & $0(1)$ & $1(1)$ \\
& & & & & & \\
\hline
\end{tabular}


Table S-5. Hydrogen coordinates ( $\left.\mathrm{x} 10^{4}\right)$ and isotropic displacement parameters $\left(\AA^{2} \times 10^{3}\right)$ for allyl(ItBu)NiCl (1d).

\begin{tabular}{|c|c|c|c|c|}
\hline & $\mathrm{x}$ & $\mathrm{y}$ & $\mathrm{z}$ & $\mathrm{U}(\mathrm{eq})$ \\
\hline $\mathrm{H}(2 \mathrm{~A})$ & $1170(30)$ & $-650(30)$ & $10(20)$ & $34(7)$ \\
\hline $\mathrm{H}(2 \mathrm{~B})$ & $190(20)$ & $260(20)$ & $-485(17)$ & $20(6)$ \\
\hline $\mathrm{H}(2 \mathrm{C})$ & $-10(20)$ & $40(30)$ & $616(18)$ & $34(7)$ \\
\hline $\mathrm{H}(3 \mathrm{~A})$ & $-100(20)$ & $2390(30)$ & $960(20)$ & $32(7)$ \\
\hline $\mathrm{H}(3 \mathrm{~B})$ & $-60(20)$ & $2870(30)$ & $-100(20)$ & $30(7)$ \\
\hline $\mathrm{H}(3 \mathrm{C})$ & $830(30)$ & $3450(30)$ & 710(19) & $33(7)$ \\
\hline $\mathrm{H}(4 \mathrm{~A})$ & $2540(20)$ & $2120(30)$ & $1240(20)$ & $33(7)$ \\
\hline $\mathrm{H}(4 \mathrm{~B})$ & $2720(30)$ & $480(40)$ & $1020(20)$ & $45(9)$ \\
\hline $\mathrm{H}(4 \mathrm{C})$ & $1630(30)$ & $1090(30)$ & $1660(20)$ & $50(9)$ \\
\hline $\mathrm{H}(5)$ & $2290(20)$ & $3880(30)$ & $-370(20)$ & $27(7)$ \\
\hline $\mathrm{H}(6)$ & $3710(20)$ & $3880(20)$ & $-1740(17)$ & $24(6)$ \\
\hline $\mathrm{H}(9 \mathrm{~A})$ & $5100(30)$ & $-330(30)$ & $-2950(20)$ & $40(8)$ \\
\hline $\mathrm{H}(9 \mathrm{~B})$ & $3990(20)$ & $-830(20)$ & $-2380(20)$ & $9(5)$ \\
\hline $\mathrm{H}(9 \mathrm{C})$ & $5070(30)$ & $-150(30)$ & $-1790(20)$ & $54(9)$ \\
\hline $\mathrm{H}(10 \mathrm{~A})$ & $5730(30)$ & $2040(30)$ & $-3140(20)$ & $42(8)$ \\
\hline $\mathrm{H}(10 \mathrm{~B})$ & $5600(40)$ & $2440(40)$ & $-2140(30)$ & $69(11)$ \\
\hline $\mathrm{H}(10 \mathrm{C})$ & $4810(30)$ & $3310(40)$ & $-2830(20)$ & $65(10)$ \\
\hline $\mathrm{H}(11 \mathrm{~A})$ & $3730(30)$ & $1120(30)$ & $-3950(20)$ & $37(7)$ \\
\hline $\mathrm{H}(11 \mathrm{~B})$ & $2610(30)$ & $830(40)$ & $-3380(30)$ & $44(11)$ \\
\hline $\mathrm{H}(11 \mathrm{C})$ & $3030(30)$ & $2520(40)$ & $-3480(20)$ & $67(10)$ \\
\hline $\mathrm{H}(12 \mathrm{~A})$ & $3200(30)$ & $-1580(30)$ & $280(20)$ & $42(7)$ \\
\hline $\mathrm{H}(12 \mathrm{~B})$ & $4400(20)$ & $-1060(20)$ & $-157(18)$ & $24(7)$ \\
\hline $\mathrm{H}(13)$ & $4260(30)$ & $-2780(30)$ & $-1410(20)$ & $57(8)$ \\
\hline $\mathrm{H}(14 \mathrm{~A})$ & $1680(30)$ & $-3150(30)$ & $-570(20)$ & $42(7)$ \\
\hline $\mathrm{H}(14 \mathrm{~B})$ & $2300(30)$ & $-3650(40)$ & $-1680(30)$ & $60(11)$ \\
\hline
\end{tabular}


Table S-6. Torsion angles $\left[{ }^{\circ}\right]$ for allyl(ItBu) $\mathrm{NiCl}(\mathbf{1 d})$.

\begin{tabular}{lc}
\hline $\mathrm{C}(7)-\mathrm{N}(1)-\mathrm{C}(1)-\mathrm{C}(4)$ & $91.2(2)$ \\
$\mathrm{C}(5)-\mathrm{N}(1)-\mathrm{C}(1)-\mathrm{C}(4)$ & $-83.6(3)$ \\
$\mathrm{C}(7)-\mathrm{N}(1)-\mathrm{C}(1)-\mathrm{C}(2)$ & $-31.3(3)$ \\
$\mathrm{C}(5)-\mathrm{N}(1)-\mathrm{C}(1)-\mathrm{C}(2)$ & $153.88(19)$ \\
$\mathrm{C}(7)-\mathrm{N}(1)-\mathrm{C}(1)-\mathrm{C}(3)$ & $-149.5(2)$ \\
$\mathrm{C}(5)-\mathrm{N}(1)-\mathrm{C}(1)-\mathrm{C}(3)$ & $35.7(3)$ \\
$\mathrm{C}(7)-\mathrm{N}(1)-\mathrm{C}(5)-\mathrm{C}(6)$ & $0.8(2)$ \\
$\mathrm{C}(1)-\mathrm{N}(1)-\mathrm{C}(5)-\mathrm{C}(6)$ & $176.38(19)$ \\
$\mathrm{N}(1)-\mathrm{C}(5)-\mathrm{C}(6)-\mathrm{N}(2)$ & $-1.3(2)$ \\
$\mathrm{C}(7)-\mathrm{N}(2)-\mathrm{C}(6)-\mathrm{C}(5)$ & $1.5(2)$ \\
$\mathrm{C}(8)-\mathrm{N}(2)-\mathrm{C}(6)-\mathrm{C}(5)$ & $176.02(19)$ \\
$\mathrm{C}(5)-\mathrm{N}(1)-\mathrm{C}(7)-\mathrm{N}(2)$ & $0.2(2)$ \\
$\mathrm{C}(1)-\mathrm{N}(1)-\mathrm{C}(7)-\mathrm{N}(2)$ & $-175.14(18)$ \\
$\mathrm{C}(5)-\mathrm{N}(1)-\mathrm{C}(7)-\mathrm{Ni}(1)$ & $-177.82(15)$ \\
$\mathrm{C}(1)-\mathrm{N}(1)-\mathrm{C}(7)-\mathrm{Ni}(1)$ & $6.9(3)$ \\
$\mathrm{C}(6)-\mathrm{N}(2)-\mathrm{C}(7)-\mathrm{N}(1)$ & $-1.0(2)$ \\
$\mathrm{C}(8)-\mathrm{N}(2)-\mathrm{C}(7)-\mathrm{N}(1)$ & $-175.14(18)$ \\
$\mathrm{C}(6)-\mathrm{N}(2)-\mathrm{C}(7)-\mathrm{Ni}(1)$ & $177.01(14)$ \\
$\mathrm{C}(8)-\mathrm{N}(2)-\mathrm{C}(7)-\mathrm{Ni}(1)$ & $2.9(3)$ \\
$\mathrm{C}(13)-\mathrm{Ni}(1)-\mathrm{C}(7)-\mathrm{N}(1)$ & $-105.32(19)$ \\
$\mathrm{C}(12)-\mathrm{Ni}(1)-\mathrm{C}(7)-\mathrm{N}(1)$ & $-83.95(19)$ \\
$\mathrm{C}(14)-\mathrm{Ni}(1)-\mathrm{C}(7)-\mathrm{N}(1)$ & $-59.2(5)$ \\
$\mathrm{Cl}(2)-\mathrm{Ni}(1)-\mathrm{C}(7)-\mathrm{N}(1)$ & $93.76(17)$ \\
$\mathrm{C}(13)-\mathrm{Ni}(1)-\mathrm{C}(7)-\mathrm{N}(2)$ & $77.1(2)$ \\
$\mathrm{C}(12)-\mathrm{Ni}(1)-\mathrm{C}(7)-\mathrm{N}(2)$ & $98.51(18)$ \\
$\mathrm{C}(14)-\mathrm{Ni}(1)-\mathrm{C}(7)-\mathrm{N}(2)$ & $123.3(5)$ \\
$\mathrm{Cl}(2)-\mathrm{Ni}(1)-\mathrm{C}(7)-\mathrm{N}(2)$ & $-83.78(16)$ \\
$\mathrm{C}(7)-\mathrm{N}(2)-\mathrm{C}(8)-\mathrm{C}(9)$ & $-35.4(3)$ \\
$\mathrm{C}(6)-\mathrm{N}(2)-\mathrm{C}(8)-\mathrm{C}(9)$ & $151.1(2)$ \\
$\mathrm{C}(7)-\mathrm{N}(2)-\mathrm{C}(8)-\mathrm{C}(11)$ & $85.6(2)$ \\
$\mathrm{C}(6)-\mathrm{N}(2)-\mathrm{C}(8)-\mathrm{C}(11)$ & $-87.9(2)$ \\
$\mathrm{C}(7)-\mathrm{N}(2)-\mathrm{C}(8)-\mathrm{C}(10)$ & $-154.2(2)$ \\
$\mathrm{C}(6)-\mathrm{N}(2)-\mathrm{C}(8)-\mathrm{C}(10)$ & $32.2(3)$ \\
$\mathrm{C}(7)-\mathrm{Ni}(1)-\mathrm{C}(12)-\mathrm{C}(13)$ & \\
$\mathrm{C}(14)-\mathrm{Ni}(1)-\mathrm{C}(12)-\mathrm{C}(13)$ & \\
$\mathrm{Cl}(2)-\mathrm{Ni}(1)-\mathrm{C}(12)-\mathrm{C}(13)$ & \\
$\mathrm{Ni}(1)-\mathrm{C}(12)-\mathrm{C}(13)-\mathrm{C}(14)$ & \\
& \\
& \\
&
\end{tabular}


C(7)-Ni(1)-C(13)-C(14)

$\mathrm{C}(12)-\mathrm{Ni}(1)-\mathrm{C}(13)-\mathrm{C}(14)$

$\mathrm{Cl}(2)-\mathrm{Ni}(1)-\mathrm{C}(13)-\mathrm{C}(14)$

$\mathrm{C}(7)-\mathrm{Ni}(1)-\mathrm{C}(13)-\mathrm{C}(12)$

$\mathrm{C}(14)-\mathrm{Ni}(1)-\mathrm{C}(13)-\mathrm{C}(12)$

$\mathrm{Cl}(2)-\mathrm{Ni}(1)-\mathrm{C}(13)-\mathrm{C}(12)$

$\mathrm{C}(12)-\mathrm{C}(13)-\mathrm{C}(14)-\mathrm{Ni}(1)$

C(7)-Ni(1)-C(14)-C(13)

$\mathrm{C}(12)-\mathrm{Ni}(1)-\mathrm{C}(14)-\mathrm{C}(13)$

$\mathrm{Cl}(2)-\mathrm{Ni}(1)-\mathrm{C}(14)-\mathrm{C}(13)$
165.73(17)

$132.3(2)$

$-38.2(2)$

$33.5(2)$

$-132.3(2)$

$-170.41(13)$

$53.7(2)$

$-56.5(5)$

$-30.62(17)$

150.33(16)

Symmetry transformations used to generate equivalent atoms: 
Table S-7. Crystal data and structure refinement for allyl( $\left.\mathrm{IBioxMe}_{4}\right) \mathrm{NiCl}(\mathbf{1 f})$.

Identification code

Empirical formula

Formula weight

Temperature

Wavelength

Crystal system

Space group

Unit cell dimensions

Volume

Z

Density (calculated)

Absorption coefficient

$\mathrm{F}(000)$

Crystal size

Theta range for data collection

Index ranges

Reflections collected

Independent reflections

Completeness to theta $=27.46^{\circ}$

Absorption correction

Max. and min. transmission

Refinement method

Data / restraints / parameters

Goodness-of-fit on F2

Final $\mathrm{R}$ indices [I $>2 \operatorname{sigma}(\mathrm{I})]$

$\mathrm{R}$ indices (all data)

Largest diff. peak and hole mss028

$\mathrm{C}_{17.50} \mathrm{H}_{25} \mathrm{ClN}_{2} \mathrm{NiO}_{2}$

389.55

150(1) K

$0.71073 \AA$

Monoclinic

$P 2{ }_{1} / C$

$\mathrm{a}=12.5757(4) \AA \quad \alpha=90^{\circ}$.

$\mathrm{b}=10.2031(2) \AA \quad \beta=113.6613(10)^{\circ}$.

$\mathrm{c}=15.6864(4) \AA \quad \gamma=90^{\circ}$.

1843.54(8) $\AA^{3}$

4

$1.404 \mathrm{Mg} / \mathrm{m} 3$

$1.208 \mathrm{~mm}-1$

820

$0.35 \times 0.28 \times 0.13 \mathrm{~mm}^{3}$

2.45 to $27.46^{\circ}$.

$-16<=\mathrm{h}<=16,-13<=\mathrm{k}<=11,-20<=\mathrm{l}<=20$

7412

$4200[\mathrm{R}(\mathrm{int})=0.0205]$

$99.5 \%$

Multi-scan

0.8587 and 0.6771

Full-matrix least-squares on F2

4200 / 2 / 260

1.047

$\mathrm{R} 1=0.0398, \mathrm{wR} 2=0.0971$

$\mathrm{R} 1=0.0543, \mathrm{wR} 2=0.1057$

0.905 and -0.717 e. $\AA^{-3}$ 
Table S-8. Atomic coordinates ( $\left.\times 10^{4}\right)$ and equivalent isotropic displacement parameters $\left(\AA^{2} \times 10^{3}\right)$ for allyl(IBioxMe 4$) \mathrm{NiCl}(\mathbf{1 f})$. U(eq) is defined as one third of the trace of the orthogonalized $\mathrm{U}^{\mathrm{ij}}$ tensor.

\begin{tabular}{|c|c|c|c|c|}
\hline & $\mathrm{x}$ & $\mathrm{y}$ & $\mathrm{z}$ & $\mathrm{U}(\mathrm{eq})$ \\
\hline $\mathrm{Ni}(1)$ & 2161(1) & $1603(1)$ & $1486(1)$ & $26(1)$ \\
\hline $\mathrm{Cl}(1)$ & $2756(1)$ & $1313(1)$ & $3004(1)$ & $35(1)$ \\
\hline $\mathrm{O}(1)$ & 491(2) & $6318(2)$ & $1417(1)$ & $37(1)$ \\
\hline $\mathrm{O}(2)$ & $3225(2)$ & $6564(2)$ & $1403(2)$ & $49(1)$ \\
\hline $\mathrm{N}(1)$ & $1163(2)$ & $4235(2)$ & $1435(1)$ & $24(1)$ \\
\hline $\mathrm{N}(2)$ & $2830(2)$ & $4384(2)$ & $1429(2)$ & $29(1)$ \\
\hline$C(1)$ & $59(2)$ & $4051(2)$ & $1543(2)$ & $31(1)$ \\
\hline$C(2)$ & $-479(2)$ & $5408(3)$ & $1169(2)$ & $34(1)$ \\
\hline$C(3)$ & $1385(2)$ & $5563(2)$ & $1417(2)$ & $29(1)$ \\
\hline $\mathrm{C}(4)$ & $2433(2)$ & $5657(2)$ & $1412(2)$ & $34(1)$ \\
\hline $\mathrm{C}(5)$ & $4308(4)$ & $5805(5)$ & $1787(4)$ & $45(1)$ \\
\hline$C(6)$ & $3991(2)$ & $4410(3)$ & $1408(2)$ & $33(1)$ \\
\hline$C(7)$ & 2061(2) & $3464(2)$ & $1453(2)$ & $24(1)$ \\
\hline $\mathrm{C}(8)$ & $306(3)$ & $3901(3)$ & $2572(2)$ & $45(1)$ \\
\hline $\mathrm{C}(9)$ & $-651(2)$ & 2927(3) & $959(2)$ & $42(1)$ \\
\hline$C(10)$ & $4782(4)$ & $3372(5)$ & $2025(4)$ & $44(1)$ \\
\hline$C(11)$ & $3802(4)$ & $4305(5)$ & 406(3) & $44(1)$ \\
\hline$C(12)$ & $1595(3)$ & $1432(3)$ & $101(2)$ & $42(1)$ \\
\hline$C(13)$ & $2212(5)$ & $309(4)$ & $546(3)$ & $37(1)$ \\
\hline$C(14)$ & 1990(4) & $-362(3)$ & $1169(3)$ & $69(1)$ \\
\hline$C\left(5^{\prime}\right)$ & $4023(6)$ & $5817(7)$ & $1007(5)$ & $37(2)$ \\
\hline$C\left(10^{\prime}\right)$ & 4894(6) & $4295(8)$ & $2410(5)$ & $42(2)$ \\
\hline$C\left(11^{\prime}\right)$ & $4229(7)$ & $3376(7)$ & $786(6)$ & $46(2)$ \\
\hline$C\left(13^{\prime}\right)$ & $1379(10)$ & 281(7) & $522(6)$ & $66(3)$ \\
\hline $\mathrm{C}(15)$ & $3407(3)$ & $5800(5)$ & 3989(3) & $82(1)$ \\
\hline$C(16)$ & $4563(3)$ & $5122(4)$ & $4734(3)$ & $52(2)$ \\
\hline$C(17)$ & $5307(3)$ & $5930(3)$ & $5432(2)$ & $78(1)$ \\
\hline$C(18)$ & $6335(3)$ & $5428(5)$ & 6094(3) & $49(2)$ \\
\hline$C(15 D)$ & $6618(4)$ & $4119(5)$ & 6056(4) & 50 \\
\hline$C(19)$ & $5875(6)$ & $3312(3)$ & $5358(5)$ & $71(2)$ \\
\hline$C(17 D)$ & $4847(5)$ & $3813(4)$ & $4696(4)$ & 50 \\
\hline
\end{tabular}


Table S-9. Bond lengths $[\AA]$ and angles $\left[{ }^{\circ}\right]$ for allyl(IBioxMe $\left.{ }_{4}\right) \mathrm{NiCl}(\mathbf{1 f})$.

\begin{tabular}{ll}
\hline $\mathrm{Ni}(1)-\mathrm{C}(7)$ & $1.902(2)$ \\
$\mathrm{Ni}(1)-\mathrm{C}\left(13^{\prime}\right)$ & $1.970(7)$ \\
$\mathrm{Ni}(1)-\mathrm{C}(13)$ & $2.000(4)$ \\
$\mathrm{Ni}(1)-\mathrm{C}(12)$ & $2.004(3)$ \\
$\mathrm{Ni}(1)-\mathrm{C}(14)$ & $2.056(3)$ \\
$\mathrm{Ni}(1)-\mathrm{Cl}(1)$ & $2.2108(7)$ \\
$\mathrm{O}(1)-\mathrm{C}(3)$ & $1.363(3)$ \\
$\mathrm{O}(1)-\mathrm{C}(2)$ & $1.456(3)$ \\
$\mathrm{O}(2)-\mathrm{C}(4)$ & $1.364(3)$ \\
$\mathrm{O}(2)-\mathrm{C}(5)$ & $1.470(5)$ \\
$\mathrm{O}(2)-\mathrm{C}\left(5^{\prime}\right)$ & $1.572(7)$ \\
$\mathrm{N}(1)-\mathrm{C}(7)$ & $1.367(3)$ \\
$\mathrm{N}(1)-\mathrm{C}(3)$ & $1.386(3)$ \\
$\mathrm{N}(1)-\mathrm{C}(1)$ & $1.478(3)$ \\
$\mathrm{N}(2)-\mathrm{C}(7)$ & $1.359(3)$ \\
$\mathrm{N}(2)-\mathrm{C}(4)$ & $1.388(3)$ \\
$\mathrm{N}(2)-\mathrm{C}(6)$ & $1.474(3)$ \\
$\mathrm{C}(1)-\mathrm{C}(9)$ & $1.515(4)$ \\
$\mathrm{C}(1)-\mathrm{C}(8)$ & $1.522(4)$ \\
$\mathrm{C}(1)-\mathrm{C}(2)$ & $1.549(3)$ \\
$\mathrm{C}(2)-\mathrm{H}(2 \mathrm{~A})$ & 0.9900 \\
$\mathrm{C}(2)-\mathrm{H}(2 \mathrm{~B})$ & 0.9900 \\
$\mathrm{C}(3)-\mathrm{C}(4)$ & $1.326(4)$ \\
$\mathrm{C}(5)-\mathrm{C}(6)$ & $1.533(5)$ \\
$\mathrm{C}(5)-\mathrm{H}(5 \mathrm{~A})$ & 0.9900 \\
$\mathrm{C}(5)-\mathrm{H}(5 \mathrm{~B})$ & 0.9900 \\
$\mathrm{C}(6)-\mathrm{C}(11)$ & $1.496(5)$ \\
$\mathrm{C}(6)-\mathrm{C}(10)$ & $1.508(5)$ \\
$\mathrm{C}(6)-\mathrm{C}\left(10^{\prime}\right)$ & $1.530(7)$ \\
$\mathrm{C}(6)-\mathrm{C}\left(11^{\prime}\right)$ & $1.545(8)$ \\
$\mathrm{C}(6)-\mathrm{C}\left(5^{\prime}\right)$ & $1.575(7)$ \\
$\mathrm{C}(8)-\mathrm{H}(8 \mathrm{~A})$ & 0.9800 \\
$\mathrm{C}(8)-\mathrm{H}(8 \mathrm{~B})$ & 0.9800 \\
$\mathrm{C}(8)-\mathrm{H}(8 \mathrm{C})$ & 0.9800 \\
$\mathrm{C}(9)-\mathrm{H}(9 \mathrm{~A})$ & 0.9800 \\
$\mathrm{C}(9)-\mathrm{H}(9 \mathrm{~B})$ & \\
$\mathrm{C}(9)-\mathrm{H}(9 \mathrm{C})$ & \\
& \\
&
\end{tabular}




\begin{tabular}{|c|c|}
\hline $\mathrm{C}(10)-\mathrm{H}(10 \mathrm{~A})$ & 0.9800 \\
\hline $\mathrm{C}(10)-\mathrm{H}(10 \mathrm{~B})$ & 0.9800 \\
\hline $\mathrm{C}(10)-\mathrm{H}(10 \mathrm{C})$ & 0.9800 \\
\hline $\mathrm{C}(11)-\mathrm{H}(11 \mathrm{~A})$ & 0.9800 \\
\hline $\mathrm{C}(11)-\mathrm{H}(11 \mathrm{~B})$ & 0.9800 \\
\hline $\mathrm{C}(11)-\mathrm{H}(11 \mathrm{C})$ & 0.9800 \\
\hline $\mathrm{C}(12)-\mathrm{C}(13)$ & $1.402(5)$ \\
\hline $\mathrm{C}(12)-\mathrm{C}\left(13^{\prime}\right)$ & $1.426(9)$ \\
\hline $\mathrm{C}(12)-\mathrm{H}(12 \mathrm{~A})$ & 0.9500 \\
\hline $\mathrm{C}(12)-\mathrm{H}(12 \mathrm{~B})$ & 0.9500 \\
\hline $\mathrm{C}(12)-\mathrm{H}(12 \mathrm{C})$ & 0.9500 \\
\hline $\mathrm{C}(12)-\mathrm{H}(12 \mathrm{D})$ & 0.9500 \\
\hline$C(13)-C(14)$ & $1.312(6)$ \\
\hline C(13)-H(13) & 0.9500 \\
\hline$C(14)-C\left(13^{\prime}\right)$ & $1.193(8)$ \\
\hline $\mathrm{C}(14)-\mathrm{H}(14 \mathrm{~A})$ & 0.9500 \\
\hline $\mathrm{C}(14)-\mathrm{H}(14 \mathrm{~B})$ & 0.9500 \\
\hline $\mathrm{C}(14)-\mathrm{H}(14 \mathrm{C})$ & 0.9500 \\
\hline $\mathrm{C}(14)-\mathrm{H}(14 \mathrm{D})$ & 0.9500 \\
\hline $\mathrm{C}\left(5^{\prime}\right)-\mathrm{H}(5 \mathrm{C})$ & 0.9900 \\
\hline $\mathrm{C}\left(5^{\prime}\right)-\mathrm{H}(5 \mathrm{D})$ & 0.9900 \\
\hline $\mathrm{C}\left(10^{\prime}\right)-\mathrm{H}(10 \mathrm{D})$ & 0.9800 \\
\hline$C\left(10^{\prime}\right)-\mathrm{H}(10 \mathrm{E})$ & 0.9800 \\
\hline $\mathrm{C}\left(10^{\prime}\right)-\mathrm{H}(10 \mathrm{~F})$ & 0.9800 \\
\hline $\mathrm{C}\left(11^{\prime}\right)-\mathrm{H}(11 \mathrm{D})$ & 0.9800 \\
\hline $\mathrm{C}\left(11^{\prime}\right)-\mathrm{H}(11 \mathrm{E})$ & 0.9800 \\
\hline $\mathrm{C}\left(11^{\prime}\right)-\mathrm{H}(11 \mathrm{~F})$ & 0.9800 \\
\hline C(13')-H(13') & 0.9500 \\
\hline$C(15)-C(16)$ & $1.611(6)$ \\
\hline $\mathrm{C}(15)-\mathrm{H}(15 \mathrm{~A})$ & 0.9800 \\
\hline $\mathrm{C}(15)-\mathrm{H}(15 \mathrm{~B})$ & 0.9800 \\
\hline $\mathrm{C}(15)-\mathrm{H}(15 \mathrm{C})$ & 0.9800 \\
\hline$C(16)-C(17)$ & 1.3900 \\
\hline$C(16)-C(17 D)$ & 1.3900 \\
\hline $\mathrm{C}(17)-\mathrm{C}(18)$ & 1.3900 \\
\hline $\mathrm{C}(17)-\mathrm{H}(17)$ & 0.9500 \\
\hline$C(18)-C(15 D)$ & 1.3900 \\
\hline $\mathrm{C}(18)-\mathrm{H}(18)$ & 0.9500 \\
\hline$C(15 D)-C(19)$ & 1.3900 \\
\hline
\end{tabular}




\begin{tabular}{|c|c|}
\hline $\mathrm{C}(15 \mathrm{D})-\mathrm{H}(15 \mathrm{D})$ & 0.9500 \\
\hline$C(19)-C(17 D)$ & 1.3900 \\
\hline C(19)-H(19) & 0.9500 \\
\hline $\mathrm{C}(17 \mathrm{D})-\mathrm{H}(17 \mathrm{D})$ & 0.9500 \\
\hline$C(7)-N i(1)-C\left(13^{\prime}\right)$ & $131.0(2)$ \\
\hline C(7)-Ni(1)-C(13) & $131.34(15)$ \\
\hline $\mathrm{C}\left(13^{\prime}\right)-\mathrm{Ni}(1)-\mathrm{C}(13)$ & $30.2(3)$ \\
\hline $\mathrm{C}(7)-\mathrm{Ni}(1)-\mathrm{C}(12)$ & $93.85(10)$ \\
\hline $\mathrm{C}\left(13^{\prime}\right)-\mathrm{Ni}(1)-\mathrm{C}(12)$ & $42.0(3)$ \\
\hline $\mathrm{C}(13)-\mathrm{Ni}(1)-\mathrm{C}(12)$ & $41.00(15)$ \\
\hline $\mathrm{C}(7)-\mathrm{Ni}(1)-\mathrm{C}(14)$ & $165.34(13)$ \\
\hline $\mathrm{C}\left(13^{\prime}\right)-\mathrm{Ni}(1)-\mathrm{C}(14)$ & $34.4(2)$ \\
\hline $\mathrm{C}(13)-\mathrm{Ni}(1)-\mathrm{C}(14)$ & $37.70(17)$ \\
\hline$C(12)-N i(1)-C(14)$ & $72.26(13)$ \\
\hline $\mathrm{C}(7)-\mathrm{Ni}(1)-\mathrm{Cl}(1)$ & $98.79(7)$ \\
\hline $\mathrm{C}\left(13^{\prime}\right)-\mathrm{Ni}(1)-\mathrm{Cl}(1)$ & $125.7(3)$ \\
\hline $\mathrm{C}(13)-\mathrm{Ni}(1)-\mathrm{Cl}(1)$ & $127.49(14)$ \\
\hline $\mathrm{C}(12)-\mathrm{Ni}(1)-\mathrm{Cl}(1)$ & $167.27(8)$ \\
\hline $\mathrm{C}(14)-\mathrm{Ni}(1)-\mathrm{Cl}(1)$ & $95.02(10)$ \\
\hline $\mathrm{C}(3)-\mathrm{O}(1)-\mathrm{C}(2)$ & $104.00(18)$ \\
\hline $\mathrm{C}(4)-\mathrm{O}(2)-\mathrm{C}(5)$ & $101.4(2)$ \\
\hline $\mathrm{C}(4)-\mathrm{O}(2)-\mathrm{C}\left(5^{\prime}\right)$ & $105.0(3)$ \\
\hline $\mathrm{C}(5)-\mathrm{O}(2)-\mathrm{C}\left(5^{\prime}\right)$ & $43.4(3)$ \\
\hline$C(7)-N(1)-C(3)$ & $113.09(19)$ \\
\hline $\mathrm{C}(7)-\mathrm{N}(1)-\mathrm{C}(1)$ & $136.97(19)$ \\
\hline $\mathrm{C}(3)-\mathrm{N}(1)-\mathrm{C}(1)$ & $109.41(18)$ \\
\hline$C(7)-N(2)-C(4)$ & $113.1(2)$ \\
\hline $\mathrm{C}(7)-\mathrm{N}(2)-\mathrm{C}(6)$ & $137.4(2)$ \\
\hline $\mathrm{C}(4)-\mathrm{N}(2)-\mathrm{C}(6)$ & $109.5(2)$ \\
\hline $\mathrm{N}(1)-\mathrm{C}(1)-\mathrm{C}(9)$ & $112.2(2)$ \\
\hline $\mathrm{N}(1)-\mathrm{C}(1)-\mathrm{C}(8)$ & $109.5(2)$ \\
\hline $\mathrm{C}(9)-\mathrm{C}(1)-\mathrm{C}(8)$ & $112.7(2)$ \\
\hline $\mathrm{N}(1)-\mathrm{C}(1)-\mathrm{C}(2)$ & $97.49(18)$ \\
\hline $\mathrm{C}(9)-\mathrm{C}(1)-\mathrm{C}(2)$ & $112.7(2)$ \\
\hline $\mathrm{C}(8)-\mathrm{C}(1)-\mathrm{C}(2)$ & $111.4(2)$ \\
\hline $\mathrm{O}(1)-\mathrm{C}(2)-\mathrm{C}(1)$ & $106.08(19)$ \\
\hline $\mathrm{O}(1)-\mathrm{C}(2)-\mathrm{H}(2 \mathrm{~A})$ & 110.5 \\
\hline $\mathrm{C}(1)-\mathrm{C}(2)-\mathrm{H}(2 \mathrm{~A})$ & 110.5 \\
\hline
\end{tabular}




\begin{tabular}{|c|c|}
\hline $\mathrm{O}(1)-\mathrm{C}(2)-\mathrm{H}(2 \mathrm{~B})$ & 110.5 \\
\hline $\mathrm{C}(1)-\mathrm{C}(2)-\mathrm{H}(2 \mathrm{~B})$ & 110.5 \\
\hline $\mathrm{H}(2 \mathrm{~A})-\mathrm{C}(2)-\mathrm{H}(2 \mathrm{~B})$ & 108.7 \\
\hline $\mathrm{C}(4)-\mathrm{C}(3)-\mathrm{O}(1)$ & $141.4(2)$ \\
\hline $\mathrm{C}(4)-\mathrm{C}(3)-\mathrm{N}(1)$ & $106.3(2)$ \\
\hline $\mathrm{O}(1)-\mathrm{C}(3)-\mathrm{N}(1)$ & $112.3(2)$ \\
\hline $\mathrm{C}(3)-\mathrm{C}(4)-\mathrm{O}(2)$ & $141.4(2)$ \\
\hline $\mathrm{C}(3)-\mathrm{C}(4)-\mathrm{N}(2)$ & $106.4(2)$ \\
\hline $\mathrm{O}(2)-\mathrm{C}(4)-\mathrm{N}(2)$ & $112.2(2)$ \\
\hline $\mathrm{O}(2)-\mathrm{C}(5)-\mathrm{C}(6)$ & $106.0(3)$ \\
\hline $\mathrm{O}(2)-\mathrm{C}(5)-\mathrm{H}(5 \mathrm{~A})$ & 110.5 \\
\hline $\mathrm{C}(6)-\mathrm{C}(5)-\mathrm{H}(5 \mathrm{~A})$ & 110.5 \\
\hline $\mathrm{O}(2)-\mathrm{C}(5)-\mathrm{H}(5 \mathrm{~B})$ & 110.5 \\
\hline $\mathrm{C}(6)-\mathrm{C}(5)-\mathrm{H}(5 \mathrm{~B})$ & 110.5 \\
\hline $\mathrm{H}(5 \mathrm{~A})-\mathrm{C}(5)-\mathrm{H}(5 \mathrm{~B})$ & 108.7 \\
\hline $\mathrm{N}(2)-\mathrm{C}(6)-\mathrm{C}(11)$ & $106.3(3)$ \\
\hline $\mathrm{N}(2)-\mathrm{C}(6)-\mathrm{C}(10)$ & $112.2(3)$ \\
\hline$C(11)-C(6)-C(10)$ & $114.2(3)$ \\
\hline $\mathrm{N}(2)-\mathrm{C}(6)-\mathrm{C}\left(10^{\prime}\right)$ & $108.0(3)$ \\
\hline $\mathrm{C}(11)-\mathrm{C}(6)-\mathrm{C}\left(10^{\prime}\right)$ & $144.4(4)$ \\
\hline $\mathrm{C}(10)-\mathrm{C}(6)-\mathrm{C}\left(10^{\prime}\right)$ & $42.3(3)$ \\
\hline $\mathrm{N}(2)-\mathrm{C}(6)-\mathrm{C}(5)$ & $96.5(2)$ \\
\hline$C(11)-C(6)-C(5)$ & $112.4(3)$ \\
\hline$C(10)-C(6)-C(5)$ & $113.6(3)$ \\
\hline$C\left(10^{\prime}\right)-C(6)-C(5)$ & $72.7(4)$ \\
\hline $\mathrm{N}(2)-\mathrm{C}(6)-\mathrm{C}\left(11^{\prime}\right)$ & $116.5(4)$ \\
\hline $\mathrm{C}(11)-\mathrm{C}(6)-\mathrm{C}\left(11^{\prime}\right)$ & $43.8(4)$ \\
\hline$C(10)-C(6)-C\left(11^{\prime}\right)$ & $71.4(4)$ \\
\hline$C\left(10^{\prime}\right)-C(6)-C\left(11^{\prime}\right)$ & $110.1(5)$ \\
\hline$C(5)-C(6)-C\left(11^{\prime}\right)$ & $142.5(4)$ \\
\hline $\mathrm{N}(2)-\mathrm{C}(6)-\mathrm{C}\left(5^{\prime}\right)$ & $102.1(3)$ \\
\hline $\mathrm{C}(11)-\mathrm{C}(6)-\mathrm{C}\left(5^{\prime}\right)$ & $70.4(4)$ \\
\hline$C(10)-C(6)-C\left(5^{\prime}\right)$ & $141.3(4)$ \\
\hline$C\left(10^{\prime}\right)-C(6)-C\left(5^{\prime}\right)$ & $110.6(4)$ \\
\hline$C(5)-C(6)-C\left(5^{\prime}\right)$ & $42.6(3)$ \\
\hline$C\left(11^{\prime}\right)-C(6)-C\left(5^{\prime}\right)$ & $109.2(4)$ \\
\hline $\mathrm{N}(2)-\mathrm{C}(7)-\mathrm{N}(1)$ & $101.13(18)$ \\
\hline $\mathrm{N}(2)-\mathrm{C}(7)-\mathrm{Ni}(1)$ & $130.54(16)$ \\
\hline $\mathrm{N}(1)-\mathrm{C}(7)-\mathrm{Ni}(1)$ & $128.33(16)$ \\
\hline
\end{tabular}




\begin{tabular}{|c|c|}
\hline $\mathrm{C}(1)-\mathrm{C}(8)-\mathrm{H}(8 \mathrm{~A})$ & 109.5 \\
\hline $\mathrm{C}(1)-\mathrm{C}(8)-\mathrm{H}(8 \mathrm{~B})$ & 109.5 \\
\hline $\mathrm{H}(8 \mathrm{~A})-\mathrm{C}(8)-\mathrm{H}(8 \mathrm{~B})$ & 109.5 \\
\hline $\mathrm{C}(1)-\mathrm{C}(8)-\mathrm{H}(8 \mathrm{C})$ & 109.5 \\
\hline $\mathrm{H}(8 \mathrm{~A})-\mathrm{C}(8)-\mathrm{H}(8 \mathrm{C})$ & 109.5 \\
\hline $\mathrm{H}(8 \mathrm{~B})-\mathrm{C}(8)-\mathrm{H}(8 \mathrm{C})$ & 109.5 \\
\hline C(1)-C(9)-H(9A) & 109.5 \\
\hline C(1)-C(9)-H(9B) & 109.5 \\
\hline $\mathrm{H}(9 \mathrm{~A})-\mathrm{C}(9)-\mathrm{H}(9 \mathrm{~B})$ & 109.5 \\
\hline $\mathrm{C}(1)-\mathrm{C}(9)-\mathrm{H}(9 \mathrm{C})$ & 109.5 \\
\hline $\mathrm{H}(9 \mathrm{~A})-\mathrm{C}(9)-\mathrm{H}(9 \mathrm{C})$ & 109.5 \\
\hline H(9B)-C(9)-H(9C) & 109.5 \\
\hline $\mathrm{C}(6)-\mathrm{C}(10)-\mathrm{H}(10 \mathrm{~A})$ & 109.5 \\
\hline $\mathrm{C}(6)-\mathrm{C}(10)-\mathrm{H}(10 \mathrm{~B})$ & 109.5 \\
\hline $\mathrm{H}(10 \mathrm{~A})-\mathrm{C}(10)-\mathrm{H}(10 \mathrm{~B})$ & 109.5 \\
\hline $\mathrm{C}(6)-\mathrm{C}(10)-\mathrm{H}(10 \mathrm{C})$ & 109.5 \\
\hline $\mathrm{H}(10 \mathrm{~A})-\mathrm{C}(10)-\mathrm{H}(10 \mathrm{C})$ & 109.5 \\
\hline $\mathrm{H}(10 \mathrm{~B})-\mathrm{C}(10)-\mathrm{H}(10 \mathrm{C})$ & 109.5 \\
\hline $\mathrm{C}(6)-\mathrm{C}(11)-\mathrm{H}(11 \mathrm{~A})$ & 109.5 \\
\hline $\mathrm{C}(6)-\mathrm{C}(11)-\mathrm{H}(11 \mathrm{~B})$ & 109.5 \\
\hline $\mathrm{H}(11 \mathrm{~A})-\mathrm{C}(11)-\mathrm{H}(11 \mathrm{~B})$ & 109.5 \\
\hline $\mathrm{C}(6)-\mathrm{C}(11)-\mathrm{H}(11 \mathrm{C})$ & 109.5 \\
\hline $\mathrm{H}(11 \mathrm{~A})-\mathrm{C}(11)-\mathrm{H}(11 \mathrm{C})$ & 109.5 \\
\hline $\mathrm{H}(11 \mathrm{~B})-\mathrm{C}(11)-\mathrm{H}(11 \mathrm{C})$ & 109.5 \\
\hline $\mathrm{C}(13)-\mathrm{C}(12)-\mathrm{C}\left(13^{\prime}\right)$ & $42.9(5)$ \\
\hline $\mathrm{C}(13)-\mathrm{C}(12)-\mathrm{Ni}(1)$ & $69.4(2)$ \\
\hline $\mathrm{C}\left(13^{\prime}\right)-\mathrm{C}(12)-\mathrm{Ni}(1)$ & $67.7(3)$ \\
\hline $\mathrm{C}(13)-\mathrm{C}(12)-\mathrm{H}(12 \mathrm{~A})$ & 120.0 \\
\hline $\mathrm{C}\left(13^{\prime}\right)-\mathrm{C}(12)-\mathrm{H}(12 \mathrm{~A})$ & 80.9 \\
\hline $\mathrm{Ni}(1)-\mathrm{C}(12)-\mathrm{H}(12 \mathrm{~A})$ & 71.2 \\
\hline $\mathrm{C}(13)-\mathrm{C}(12)-\mathrm{H}(12 \mathrm{~B})$ & 120.0 \\
\hline $\mathrm{C}\left(13^{\prime}\right)-\mathrm{C}(12)-\mathrm{H}(12 \mathrm{~B})$ & 153.1 \\
\hline Ni(1)-C(12)-H(12B) & 132.3 \\
\hline $\mathrm{H}(12 \mathrm{~A})-\mathrm{C}(12)-\mathrm{H}(12 \mathrm{~B})$ & 120.0 \\
\hline $\mathrm{C}(13)-\mathrm{C}(12)-\mathrm{H}(12 \mathrm{C})$ & 82.9 \\
\hline $\mathrm{C}\left(13^{\prime}\right)-\mathrm{C}(12)-\mathrm{H}(12 \mathrm{C})$ & 120.0 \\
\hline $\mathrm{Ni}(1)-\mathrm{C}(12)-\mathrm{H}(12 \mathrm{C})$ & 70.6 \\
\hline $\mathrm{H}(12 \mathrm{~A})-\mathrm{C}(12)-\mathrm{H}(12 \mathrm{C})$ & 122.9 \\
\hline $\mathrm{H}(12 \mathrm{~B})-\mathrm{C}(12)-\mathrm{H}(12 \mathrm{C})$ & 65.2 \\
\hline
\end{tabular}




\begin{tabular}{|c|c|}
\hline $\mathrm{C}(13)-\mathrm{C}(12)-\mathrm{H}(12 \mathrm{D})$ & 148.9 \\
\hline $\mathrm{C}\left(13^{\prime}\right)-\mathrm{C}(12)-\mathrm{H}(12 \mathrm{D})$ & 120.0 \\
\hline $\mathrm{Ni}(1)-\mathrm{C}(12)-\mathrm{H}(12 \mathrm{D})$ & 135.3 \\
\hline $\mathrm{H}(12 \mathrm{~A})-\mathrm{C}(12)-\mathrm{H}(12 \mathrm{D})$ & 67.4 \\
\hline $\mathrm{H}(12 \mathrm{~B})-\mathrm{C}(12)-\mathrm{H}(12 \mathrm{D})$ & 61.8 \\
\hline $\mathrm{H}(12 \mathrm{C})-\mathrm{C}(12)-\mathrm{H}(12 \mathrm{D})$ & 120.0 \\
\hline $\mathrm{C}(14)-\mathrm{C}(13)-\mathrm{C}(12)$ & $123.8(4)$ \\
\hline $\mathrm{C}(14)-\mathrm{C}(13)-\mathrm{Ni}(1)$ & $73.5(2)$ \\
\hline $\mathrm{C}(12)-\mathrm{C}(13)-\mathrm{Ni}(1)$ & $69.6(2)$ \\
\hline $\mathrm{C}(14)-\mathrm{C}(13)-\mathrm{H}(13)$ & 118.1 \\
\hline $\mathrm{C}(12)-\mathrm{C}(13)-\mathrm{H}(13)$ & 118.1 \\
\hline $\mathrm{Ni}(1)-\mathrm{C}(13)-\mathrm{H}(13)$ & 132.2 \\
\hline$C\left(13^{\prime}\right)-C(14)-C(13)$ & $48.5(6)$ \\
\hline $\mathrm{C}\left(13^{\prime}\right)-\mathrm{C}(14)-\mathrm{Ni}(1)$ & $68.8(4)$ \\
\hline $\mathrm{C}(13)-\mathrm{C}(14)-\mathrm{Ni}(1)$ & $68.8(2)$ \\
\hline $\mathrm{C}\left(13^{\prime}\right)-\mathrm{C}(14)-\mathrm{H}(14 \mathrm{~A})$ & 76.5 \\
\hline $\mathrm{C}(13)-\mathrm{C}(14)-\mathrm{H}(14 \mathrm{~A})$ & 120.0 \\
\hline $\mathrm{Ni}(1)-\mathrm{C}(14)-\mathrm{H}(14 \mathrm{~A})$ & 70.5 \\
\hline$C\left(13^{\prime}\right)-C(14)-H(14 B)$ & 153.7 \\
\hline $\mathrm{C}(13)-\mathrm{C}(14)-\mathrm{H}(14 \mathrm{~B})$ & 120.0 \\
\hline $\mathrm{Ni}(1)-\mathrm{C}(14)-\mathrm{H}(14 \mathrm{~B})$ & 133.9 \\
\hline $\mathrm{H}(14 \mathrm{~A})-\mathrm{C}(14)-\mathrm{H}(14 \mathrm{~B})$ & 120.0 \\
\hline $\mathrm{C}\left(13^{\prime}\right)-\mathrm{C}(14)-\mathrm{H}(14 \mathrm{C})$ & 120.0 \\
\hline $\mathrm{C}(13)-\mathrm{C}(14)-\mathrm{H}(14 \mathrm{C})$ & 77.6 \\
\hline Ni(1)-C(14)-H(14C) & 68.4 \\
\hline $\mathrm{H}(14 \mathrm{~A})-\mathrm{C}(14)-\mathrm{H}(14 \mathrm{C})$ & 123.7 \\
\hline $\mathrm{H}(14 \mathrm{~B})-\mathrm{C}(14)-\mathrm{H}(14 \mathrm{C})$ & 70.1 \\
\hline $\mathrm{C}\left(13^{\prime}\right)-\mathrm{C}(14)-\mathrm{H}(14 \mathrm{D})$ & 120.0 \\
\hline $\mathrm{C}(13)-\mathrm{C}(14)-\mathrm{H}(14 \mathrm{D})$ & 151.3 \\
\hline Ni(1)-C(14)-H(14D) & 136.8 \\
\hline $\mathrm{H}(14 \mathrm{~A})-\mathrm{C}(14)-\mathrm{H}(14 \mathrm{D})$ & 71.2 \\
\hline $\mathrm{H}(14 \mathrm{~B})-\mathrm{C}(14)-\mathrm{H}(14 \mathrm{D})$ & 56.3 \\
\hline $\mathrm{H}(14 \mathrm{C})-\mathrm{C}(14)-\mathrm{H}(14 \mathrm{D})$ & 120.0 \\
\hline $\mathrm{O}(2)-\mathrm{C}\left(5^{\prime}\right)-\mathrm{C}(6)$ & $99.3(4)$ \\
\hline $\mathrm{O}(2)-\mathrm{C}\left(5^{\prime}\right)-\mathrm{H}(5 \mathrm{C})$ & 111.9 \\
\hline $\mathrm{C}(6)-\mathrm{C}\left(5^{\prime}\right)-\mathrm{H}(5 \mathrm{C})$ & 111.9 \\
\hline $\mathrm{O}(2)-\mathrm{C}\left(5^{\prime}\right)-\mathrm{H}(5 \mathrm{D})$ & 111.9 \\
\hline $\mathrm{C}(6)-\mathrm{C}\left(5^{\prime}\right)-\mathrm{H}(5 \mathrm{D})$ & 111.9 \\
\hline $\mathrm{H}(5 \mathrm{C})-\mathrm{C}\left(5^{\prime}\right)-\mathrm{H}(5 \mathrm{D})$ & 109.6 \\
\hline
\end{tabular}




\begin{tabular}{|c|c|}
\hline$C(6)-C\left(10^{\prime}\right)-H(10 D)$ & 109.5 \\
\hline $\mathrm{C}(6)-\mathrm{C}\left(10^{\prime}\right)-\mathrm{H}(10 \mathrm{E})$ & 109.5 \\
\hline $\mathrm{H}(10 \mathrm{D})-\mathrm{C}\left(10^{\prime}\right)-\mathrm{H}(10 \mathrm{E})$ & 109.5 \\
\hline $\mathrm{C}(6)-\mathrm{C}\left(10^{\prime}\right)-\mathrm{H}(10 \mathrm{~F})$ & 109.5 \\
\hline $\mathrm{H}(10 \mathrm{D})-\mathrm{C}\left(10^{\prime}\right)-\mathrm{H}(10 \mathrm{~F})$ & 109.5 \\
\hline $\mathrm{H}(10 \mathrm{E})-\mathrm{C}\left(10^{\prime}\right)-\mathrm{H}(10 \mathrm{~F})$ & 109.5 \\
\hline $\mathrm{C}(6)-\mathrm{C}\left(11^{\prime}\right)-\mathrm{H}(11 \mathrm{D})$ & 109.5 \\
\hline $\mathrm{C}(6)-\mathrm{C}\left(11^{\prime}\right)-\mathrm{H}(11 \mathrm{E})$ & 109.5 \\
\hline $\mathrm{H}(11 \mathrm{D})-\mathrm{C}\left(11^{\prime}\right)-\mathrm{H}(11 \mathrm{E})$ & 109.5 \\
\hline $\mathrm{C}(6)-\mathrm{C}\left(11^{\prime}\right)-\mathrm{H}(11 \mathrm{~F})$ & 109.5 \\
\hline $\mathrm{H}(11 \mathrm{D})-\mathrm{C}\left(11^{\prime}\right)-\mathrm{H}(11 \mathrm{~F})$ & 109.5 \\
\hline $\mathrm{H}(11 \mathrm{E})-\mathrm{C}\left(11^{\prime}\right)-\mathrm{H}(11 \mathrm{~F})$ & 109.5 \\
\hline$C(14)-C\left(13^{\prime}\right)-C(12)$ & $132.0(9)$ \\
\hline $\mathrm{C}(14)-\mathrm{C}\left(13^{\prime}\right)-\mathrm{Ni}(1)$ & $76.8(4)$ \\
\hline $\mathrm{C}(12)-\mathrm{C}\left(13^{\prime}\right)-\mathrm{Ni}(1)$ & $70.2(3)$ \\
\hline $\mathrm{C}(14)-\mathrm{C}\left(13^{\prime}\right)-\mathrm{H}\left(13^{\prime}\right)$ & 114.0 \\
\hline $\mathrm{C}(12)-\mathrm{C}\left(13^{\prime}\right)-\mathrm{H}\left(13^{\prime}\right)$ & 114.0 \\
\hline Ni(1)-C(13')-H(13') & 134.1 \\
\hline $\mathrm{C}(16)-\mathrm{C}(15)-\mathrm{H}(15 \mathrm{~A})$ & 109.5 \\
\hline $\mathrm{C}(16)-\mathrm{C}(15)-\mathrm{H}(15 \mathrm{~B})$ & 109.5 \\
\hline $\mathrm{H}(15 \mathrm{~A})-\mathrm{C}(15)-\mathrm{H}(15 \mathrm{~B})$ & 109.5 \\
\hline $\mathrm{C}(16)-\mathrm{C}(15)-\mathrm{H}(15 \mathrm{C})$ & 109.5 \\
\hline $\mathrm{H}(15 \mathrm{~A})-\mathrm{C}(15)-\mathrm{H}(15 \mathrm{C})$ & 109.5 \\
\hline $\mathrm{H}(15 \mathrm{~B})-\mathrm{C}(15)-\mathrm{H}(15 \mathrm{C})$ & 109.5 \\
\hline$C(17)-C(16)-C(17 D)$ & 120.0 \\
\hline$C(17)-C(16)-C(15)$ & 116.3(3) \\
\hline$C(17 D)-C(16)-C(15)$ & $123.7(3)$ \\
\hline $\mathrm{C}(18)-\mathrm{C}(17)-\mathrm{C}(16)$ & 120.0 \\
\hline $\mathrm{C}(18)-\mathrm{C}(17)-\mathrm{H}(17)$ & 120.0 \\
\hline $\mathrm{C}(16)-\mathrm{C}(17)-\mathrm{H}(17)$ & 120.0 \\
\hline$C(15 D)-C(18)-C(17)$ & 120.0 \\
\hline $\mathrm{C}(15 \mathrm{D})-\mathrm{C}(18)-\mathrm{H}(18)$ & 120.0 \\
\hline $\mathrm{C}(17)-\mathrm{C}(18)-\mathrm{H}(18)$ & 120.0 \\
\hline$C(18)-C(15 D)-C(19)$ & 120.0 \\
\hline $\mathrm{C}(18)-\mathrm{C}(15 \mathrm{D})-\mathrm{H}(15 \mathrm{D})$ & 120.0 \\
\hline $\mathrm{C}(19)-\mathrm{C}(15 \mathrm{D})-\mathrm{H}(15 \mathrm{D})$ & 120.0 \\
\hline $\mathrm{C}(17 \mathrm{D})-\mathrm{C}(19)-\mathrm{C}(15 \mathrm{D})$ & 120.0 \\
\hline $\mathrm{C}(17 \mathrm{D})-\mathrm{C}(19)-\mathrm{H}(19)$ & 120.0 \\
\hline $\mathrm{C}(15 \mathrm{D})-\mathrm{C}(19)-\mathrm{H}(19)$ & 120.0 \\
\hline
\end{tabular}


$\mathrm{C}(19)-\mathrm{C}(17 \mathrm{D})-\mathrm{C}(16) \quad 120.0$

$\mathrm{C}(19)-\mathrm{C}(17 \mathrm{D})-\mathrm{H}(17 \mathrm{D}) \quad 120.0$

C(16)-C(17D)-H(17D) $\quad 120.0$

Symmetry transformations used to generate equivalent atoms: 
Table S-10. Anisotropic displacement parameters $\left(\AA^{2} \times 10^{3}\right)$ for allyl(IBioxMe 4$) \mathrm{NiCl}(\mathbf{1 f})$. The anisotropic displacement factor exponent takes the form: $-2 \pi^{2}\left[h^{2} a^{* 2} U^{11}+\ldots+2 h k a^{*} b^{*} U^{12}\right]$

\begin{tabular}{|c|c|c|c|c|c|c|}
\hline & $\mathrm{U}^{11}$ & $\mathrm{U}^{22}$ & $\mathrm{U}^{33}$ & $\mathrm{U}^{23}$ & $\mathrm{U}^{13}$ & $\mathrm{U}^{12}$ \\
\hline $\mathrm{Ni}(1)$ & $26(1)$ & $22(1)$ & $31(1)$ & 1(1) & 12(1) & $2(1)$ \\
\hline $\mathrm{Cl}(1)$ & $34(1)$ & $40(1)$ & $34(1)$ & $7(1)$ & $15(1)$ & $3(1)$ \\
\hline $\mathrm{O}(1)$ & $32(1)$ & $31(1)$ & $47(1)$ & $-3(1)$ & $15(1)$ & $9(1)$ \\
\hline $\mathrm{O}(2)$ & $30(1)$ & $27(1)$ & $84(2)$ & $4(1)$ & $16(1)$ & $-6(1)$ \\
\hline $\mathrm{N}(1)$ & $23(1)$ & $24(1)$ & $28(1)$ & $2(1)$ & $12(1)$ & $2(1)$ \\
\hline $\mathrm{N}(2)$ & $22(1)$ & $24(1)$ & $42(1)$ & $3(1)$ & 11(1) & $1(1)$ \\
\hline $\mathrm{C}(1)$ & $25(1)$ & $37(1)$ & $36(1)$ & $3(1)$ & $17(1)$ & $4(1)$ \\
\hline $\mathrm{C}(2)$ & $27(1)$ & $38(1)$ & $38(1)$ & $2(1)$ & $15(1)$ & $7(1)$ \\
\hline$C(3)$ & $29(1)$ & $22(1)$ & $34(1)$ & $-1(1)$ & $10(1)$ & $5(1)$ \\
\hline $\mathrm{C}(4)$ & $26(1)$ & $26(1)$ & $44(2)$ & $1(1)$ & $6(1)$ & $1(1)$ \\
\hline$C(5)$ & $28(2)$ & $47(3)$ & $62(4)$ & $2(2)$ & $19(2)$ & $-11(2)$ \\
\hline$C(6)$ & $23(1)$ & $38(1)$ & $40(1)$ & $8(1)$ & $14(1)$ & $2(1)$ \\
\hline$C(7)$ & $23(1)$ & $25(1)$ & $24(1)$ & $2(1)$ & $9(1)$ & $2(1)$ \\
\hline $\mathrm{C}(8)$ & $47(2)$ & $55(2)$ & $43(2)$ & $8(1)$ & $29(1)$ & 11(1) \\
\hline $\mathrm{C}(9)$ & $30(1)$ & $40(2)$ & $56(2)$ & $1(1)$ & $19(1)$ & $-3(1)$ \\
\hline$C(10)$ & $23(2)$ & $58(3)$ & $50(3)$ & $8(2)$ & $14(2)$ & $9(2)$ \\
\hline $\mathrm{C}(11)$ & $35(3)$ & $59(3)$ & $47(3)$ & $9(2)$ & $25(2)$ & $-3(2)$ \\
\hline$C(12)$ & $55(2)$ & $37(2)$ & $31(1)$ & $-6(1)$ & $13(1)$ & $2(1)$ \\
\hline$C(13)$ & $43(3)$ & $34(2)$ & $37(3)$ & $-7(2)$ & $19(2)$ & $3(2)$ \\
\hline$C(14)$ & $125(4)$ & $25(2)$ & $56(2)$ & $-8(1)$ & $36(2)$ & $0(2)$ \\
\hline$C\left(5^{\prime}\right)$ & $28(3)$ & $43(4)$ & $42(4)$ & $14(3)$ & $16(3)$ & $5(3)$ \\
\hline $\mathrm{C}\left(10^{\prime}\right)$ & $30(4)$ & $44(4)$ & $48(4)$ & $9(3)$ & $13(3)$ & $0(3)$ \\
\hline$C\left(11^{\prime}\right)$ & $47(4)$ & $43(4)$ & $62(5)$ & $2(4)$ & $35(4)$ & $9(3)$ \\
\hline$C\left(13^{\prime}\right)$ & $91(8)$ & $34(4)$ & $44(5)$ & $-15(4)$ & $-3(5)$ & $0(5)$ \\
\hline$C(15)$ & $52(2)$ & $123(4)$ & $69(3)$ & $-6(3)$ & $22(2)$ & $-10(2)$ \\
\hline$C(16)$ & $54(4)$ & $67(4)$ & $38(4)$ & $-15(3)$ & $22(3)$ & $-25(4)$ \\
\hline $\mathrm{C}(17)$ & $79(3)$ & $98(3)$ & $75(3)$ & $-46(2)$ & $51(2)$ & $-49(3)$ \\
\hline$C(18)$ & $48(4)$ & $62(4)$ & $36(3)$ & $-21(3)$ & $17(3)$ & $-21(3)$ \\
\hline$C(19)$ & $92(6)$ & $44(4)$ & $90(6)$ & $-25(4)$ & $51(5)$ & $-13(4)$ \\
\hline
\end{tabular}


Table S-11. Hydrogen coordinates $\left(\times 10^{4}\right)$ and isotropic displacement parameters $\left(\AA^{2} \times 10^{3}\right)$ for allyl(IBioxMe $\mathrm{ISiCl}_{4}$ (1f).

\begin{tabular}{|c|c|c|c|c|}
\hline & $\mathrm{x}$ & $\mathrm{y}$ & $\mathrm{z}$ & $\mathrm{U}(\mathrm{eq})$ \\
\hline $\mathrm{H}(2 \mathrm{~A})$ & -916 & 5377 & 485 & 41 \\
\hline $\mathrm{H}(2 \mathrm{~B})$ & -1016 & 5679 & 1457 & 41 \\
\hline $\mathrm{H}(5 \mathrm{~A})$ & 4626 & 5804 & 2476 & 54 \\
\hline $\mathrm{H}(5 \mathrm{~B})$ & 4896 & 6184 & 1587 & 54 \\
\hline $\mathrm{H}(8 \mathrm{~A})$ & 786 & 3121 & 2819 & 67 \\
\hline $\mathrm{H}(8 \mathrm{~B})$ & -428 & 3808 & 2646 & 67 \\
\hline $\mathrm{H}(8 \mathrm{C})$ & 720 & 4678 & 2911 & 67 \\
\hline $\mathrm{H}(9 \mathrm{~A})$ & -730 & 3031 & 315 & 63 \\
\hline $\mathrm{H}(9 \mathrm{~B})$ & -1423 & 2927 & 974 & 63 \\
\hline $\mathrm{H}(9 \mathrm{C})$ & -260 & 2096 & 1209 & 63 \\
\hline $\mathrm{H}(10 \mathrm{~A})$ & 4863 & 3505 & 2667 & 65 \\
\hline $\mathrm{H}(10 \mathrm{~B})$ & 5546 & 3435 & 2000 & 65 \\
\hline $\mathrm{H}(10 \mathrm{C})$ & 4450 & 2504 & 1810 & 65 \\
\hline $\mathrm{H}(11 \mathrm{~A})$ & 3280 & 5006 & 50 & 66 \\
\hline $\mathrm{H}(11 \mathrm{~B})$ & 3455 & 3453 & 161 & 66 \\
\hline $\mathrm{H}(11 \mathrm{C})$ & 4548 & 4388 & 350 & 66 \\
\hline $\mathrm{H}(12 \mathrm{~A})$ & 977 & 1747 & 247 & 50 \\
\hline $\mathrm{H}(12 \mathrm{~B})$ & 1796 & 1877 & -345 & 50 \\
\hline $\mathrm{H}(12 \mathrm{C})$ & 2346 & 1817 & 343 & 50 \\
\hline $\mathrm{H}(12 \mathrm{D})$ & 989 & 1814 & -420 & 50 \\
\hline $\mathrm{H}(13)$ & 2826 & 17 & 386 & 45 \\
\hline $\mathrm{H}(14 \mathrm{~A})$ & 1381 & -93 & 1342 & 83 \\
\hline $\mathrm{H}(14 \mathrm{~B})$ & 2438 & -1116 & 1447 & 83 \\
\hline $\mathrm{H}(14 \mathrm{C})$ & 2780 & -123 & 1501 & 83 \\
\hline $\mathrm{H}(14 \mathrm{D})$ & 1687 & -1112 & 1352 & 83 \\
\hline $\mathrm{H}(5 \mathrm{C})$ & 4819 & 6182 & 1250 & 45 \\
\hline $\mathrm{H}(5 \mathrm{D})$ & 3692 & 5822 & 318 & 45 \\
\hline $\mathrm{H}(10 \mathrm{D})$ & 4735 & 4954 & 2798 & 62 \\
\hline $\mathrm{H}(10 \mathrm{E})$ & 5671 & 4440 & 2424 & 62 \\
\hline $\mathrm{H}(10 \mathrm{~F})$ & 4855 & 3418 & 2650 & 62 \\
\hline $\mathrm{H}(11 \mathrm{D})$ & 3649 & 3461 & 146 & 70 \\
\hline $\mathrm{H}(11 \mathrm{E})$ & 4182 & 2496 & 1020 & 70 \\
\hline $\mathrm{H}(11 \mathrm{~F})$ & 5006 & 3514 & 799 & 70 \\
\hline
\end{tabular}




$\begin{array}{lrrrr}\mathrm{H}\left(13^{\prime}\right) & 604 & -33 & 234 & 79 \\ \mathrm{H}(15 \mathrm{~A}) & 2952 & 5148 & 3528 & 123 \\ \mathrm{H}(15 \mathrm{~B}) & 3623 & 6517 & 3674 & 123 \\ \mathrm{H}(15 \mathrm{C}) & 2941 & 6147 & 4311 & 123 \\ \mathrm{H}(17) & 5113 & 6825 & 5458 & 93 \\ \mathrm{H}(18) & 6843 & 5980 & 6571 & 58 \\ \mathrm{H}(15 \mathrm{D}) & 7321 & 3776 & 6508 & 60 \\ \mathrm{H}(19) & 6069 & 2417 & 5332 & 85 \\ \mathrm{H}(17 \mathrm{D}) & 4339 & 3261 & 4219 & 60 \\ \end{array}$


Table S-12. Torsion angles $\left[{ }^{\circ}\right]$ for allyl(IBioxMe 4$) \operatorname{NiCl}(\mathbf{1 f})$.

\begin{tabular}{lc}
\hline $\mathrm{C}(7)-\mathrm{N}(1)-\mathrm{C}(1)-\mathrm{C}(9)$ & $-48.5(4)$ \\
$\mathrm{C}(3)-\mathrm{N}(1)-\mathrm{C}(1)-\mathrm{C}(9)$ & $140.8(2)$ \\
$\mathrm{C}(7)-\mathrm{N}(1)-\mathrm{C}(1)-\mathrm{C}(8)$ & $77.4(3)$ \\
$\mathrm{C}(3)-\mathrm{N}(1)-\mathrm{C}(1)-\mathrm{C}(8)$ & $-93.3(2)$ \\
$\mathrm{C}(7)-\mathrm{N}(1)-\mathrm{C}(1)-\mathrm{C}(2)$ & $-166.7(3)$ \\
$\mathrm{C}(3)-\mathrm{N}(1)-\mathrm{C}(1)-\mathrm{C}(2)$ & $22.5(2)$ \\
$\mathrm{C}(3)-\mathrm{O}(1)-\mathrm{C}(2)-\mathrm{C}(1)$ & $30.1(2)$ \\
$\mathrm{N}(1)-\mathrm{C}(1)-\mathrm{C}(2)-\mathrm{O}(1)$ & $-31.6(2)$ \\
$\mathrm{C}(9)-\mathrm{C}(1)-\mathrm{C}(2)-\mathrm{O}(1)$ & $-149.6(2)$ \\
$\mathrm{C}(8)-\mathrm{C}(1)-\mathrm{C}(2)-\mathrm{O}(1)$ & $82.7(2)$ \\
$\mathrm{C}(2)-\mathrm{O}(1)-\mathrm{C}(3)-\mathrm{C}(4)$ & $164.9(4)$ \\
$\mathrm{C}(2)-\mathrm{O}(1)-\mathrm{C}(3)-\mathrm{N}(1)$ & $-15.8(3)$ \\
$\mathrm{C}(7)-\mathrm{N}(1)-\mathrm{C}(3)-\mathrm{C}(4)$ & $0.8(3)$ \\
$\mathrm{C}(1)-\mathrm{N}(1)-\mathrm{C}(3)-\mathrm{C}(4)$ & $173.9(2)$ \\
$\mathrm{C}(7)-\mathrm{N}(1)-\mathrm{C}(3)-\mathrm{O}(1)$ & $-178.74(19)$ \\
$\mathrm{C}(1)-\mathrm{N}(1)-\mathrm{C}(3)-\mathrm{O}(1)$ & $-5.6(3)$ \\
$\mathrm{O}(1)-\mathrm{C}(3)-\mathrm{C}(4)-\mathrm{O}(2)$ & $-0.2(7)$ \\
$\mathrm{N}(1)-\mathrm{C}(3)-\mathrm{C}(4)-\mathrm{O}(2)$ & $-179.5(3)$ \\
$\mathrm{O}(1)-\mathrm{C}(3)-\mathrm{C}(4)-\mathrm{N}(2)$ & $179.2(3)$ \\
$\mathrm{N}(1)-\mathrm{C}(3)-\mathrm{C}(4)-\mathrm{N}(2)$ & $-0.1(3)$ \\
$\mathrm{C}(5)-\mathrm{O}(2)-\mathrm{C}(4)-\mathrm{C}(3)$ & $157.5(4)$ \\
$\mathrm{C}\left(5^{\prime}\right)-\mathrm{O}(2)-\mathrm{C}(4)-\mathrm{C}(3)$ & $-158.0(4)$ \\
$\mathrm{C}(5)-\mathrm{O}(2)-\mathrm{C}(4)-\mathrm{N}(2)$ & $-21.8(4)$ \\
$\mathrm{C}\left(5^{\prime}\right)-\mathrm{O}(2)-\mathrm{C}(4)-\mathrm{N}(2)$ & $22.7(4)$ \\
$\mathrm{C}(7)-\mathrm{N}(2)-\mathrm{C}(4)-\mathrm{C}(3)$ & $-0.6(3)$ \\
$\mathrm{C}(6)-\mathrm{N}(2)-\mathrm{C}(4)-\mathrm{C}(3)$ & $179.0(2)$ \\
$\mathrm{C}(7)-\mathrm{N}(2)-\mathrm{C}(4)-\mathrm{O}(2)$ & $179.0(2)$ \\
$\mathrm{C}(6)-\mathrm{N}(2)-\mathrm{C}(4)-\mathrm{O}(2)$ & $-1.4(3)$ \\
$\mathrm{C}(4)-\mathrm{O}(2)-\mathrm{C}(5)-\mathrm{C}(6)$ & $36.4(4)$ \\
$\mathrm{C}\left(5^{\prime}\right)-\mathrm{O}(2)-\mathrm{C}(5)-\mathrm{C}(6)$ & $-63.5(5)$ \\
$\mathrm{C}(7)-\mathrm{N}(2)-\mathrm{C}(6)-\mathrm{C}(11)$ & $86.4(4)$ \\
$\mathrm{C}(4)-\mathrm{N}(2)-\mathrm{C}(6)-\mathrm{C}(11)$ & $-93.1(3)$ \\
$\mathrm{C}(7)-\mathrm{N}(2)-\mathrm{C}(6)-\mathrm{C}(10)$ & $-39.1(5)$ \\
$\mathrm{C}(4)-\mathrm{N}(2)-\mathrm{C}(6)-\mathrm{C}(10)$ & $141.4(3)$ \\
$\mathrm{C}(7)-\mathrm{N}(2)-\mathrm{C}(6)-\mathrm{C}\left(10^{\prime}\right)$ & $-84.1(5)$ \\
$\mathrm{C}(4)-\mathrm{N}(2)-\mathrm{C}(6)-\mathrm{C}\left(10^{\prime}\right)$ & \\
$\mathrm{C}(7)-\mathrm{N}(2)-\mathrm{C}(6)-\mathrm{C}(5)$ & \\
& \\
& \\
&
\end{tabular}


$\mathrm{C}(4)-\mathrm{N}(2)-\mathrm{C}(6)-\mathrm{C}(5)$

$\mathrm{C}(7)-\mathrm{N}(2)-\mathrm{C}(6)-\mathrm{C}\left(11^{\prime}\right)$

$\mathrm{C}(4)-\mathrm{N}(2)-\mathrm{C}(6)-\mathrm{C}\left(11^{\prime}\right)$

$\mathrm{C}(7)-\mathrm{N}(2)-\mathrm{C}(6)-\mathrm{C}\left(5^{\prime}\right)$

$\mathrm{C}(4)-\mathrm{N}(2)-\mathrm{C}(6)-\mathrm{C}\left(5^{\prime}\right)$

$\mathrm{O}(2)-\mathrm{C}(5)-\mathrm{C}(6)-\mathrm{N}(2)$

$\mathrm{O}(2)-\mathrm{C}(5)-\mathrm{C}(6)-\mathrm{C}(11)$

$\mathrm{O}(2)-\mathrm{C}(5)-\mathrm{C}(6)-\mathrm{C}(10)$

$\mathrm{O}(2)-\mathrm{C}(5)-\mathrm{C}(6)-\mathrm{C}\left(10^{\prime}\right)$

$\mathrm{O}(2)-\mathrm{C}(5)-\mathrm{C}(6)-\mathrm{C}\left(11^{\prime}\right)$

$\mathrm{O}(2)-\mathrm{C}(5)-\mathrm{C}(6)-\mathrm{C}\left(5^{\prime}\right)$

$\mathrm{C}(4)-\mathrm{N}(2)-\mathrm{C}(7)-\mathrm{N}(1)$

$\mathrm{C}(6)-\mathrm{N}(2)-\mathrm{C}(7)-\mathrm{N}(1)$

$\mathrm{C}(4)-\mathrm{N}(2)-\mathrm{C}(7)-\mathrm{Ni}(1)$

$\mathrm{C}(6)-\mathrm{N}(2)-\mathrm{C}(7)-\mathrm{Ni}(1)$

$\mathrm{C}(3)-\mathrm{N}(1)-\mathrm{C}(7)-\mathrm{N}(2)$

$\mathrm{C}(1)-\mathrm{N}(1)-\mathrm{C}(7)-\mathrm{N}(2)$

$\mathrm{C}(3)-\mathrm{N}(1)-\mathrm{C}(7)-\mathrm{Ni}(1)$

$\mathrm{C}(1)-\mathrm{N}(1)-\mathrm{C}(7)-\mathrm{Ni}(1)$

$\mathrm{C}\left(13^{\prime}\right)-\mathrm{Ni}(1)-\mathrm{C}(7)-\mathrm{N}(2)$

$\mathrm{C}(13)-\mathrm{Ni}(1)-\mathrm{C}(7)-\mathrm{N}(2)$

$\mathrm{C}(12)-\mathrm{Ni}(1)-\mathrm{C}(7)-\mathrm{N}(2)$

$\mathrm{C}(14)-\mathrm{Ni}(1)-\mathrm{C}(7)-\mathrm{N}(2)$

$\mathrm{Cl}(1)-\mathrm{Ni}(1)-\mathrm{C}(7)-\mathrm{N}(2)$

C(13')-Ni(1)-C(7)-N(1)

$\mathrm{C}(13)-\mathrm{Ni}(1)-\mathrm{C}(7)-\mathrm{N}(1)$

$\mathrm{C}(12)-\mathrm{Ni}(1)-\mathrm{C}(7)-\mathrm{N}(1)$

C(14)-Ni(1)-C(7)-N(1)

$\mathrm{Cl}(1)-\mathrm{Ni}(1)-\mathrm{C}(7)-\mathrm{N}(1)$

$\mathrm{C}(7)-\mathrm{Ni}(1)-\mathrm{C}(12)-\mathrm{C}(13)$

$\mathrm{C}\left(13^{\prime}\right)-\mathrm{Ni}(1)-\mathrm{C}(12)-\mathrm{C}(13)$

$\mathrm{C}(14)-\mathrm{Ni}(1)-\mathrm{C}(12)-\mathrm{C}(13)$

$\mathrm{Cl}(1)-\mathrm{Ni}(1)-\mathrm{C}(12)-\mathrm{C}(13)$

$\mathrm{C}(7)-\mathrm{Ni}(1)-\mathrm{C}(12)-\mathrm{C}\left(13^{\prime}\right)$

$\mathrm{C}(13)-\mathrm{Ni}(1)-\mathrm{C}(12)-\mathrm{C}\left(13^{\prime}\right)$

$\mathrm{C}(14)-\mathrm{Ni}(1)-\mathrm{C}(12)-\mathrm{C}\left(13^{\prime}\right)$

$\mathrm{Cl}(1)-\mathrm{Ni}(1)-\mathrm{C}(12)-\mathrm{C}\left(13^{\prime}\right)$

$\mathrm{C}\left(13^{\prime}\right)-\mathrm{C}(12)-\mathrm{C}(13)-\mathrm{C}(14)$

Ni(1)-C(12)-C(13)-C(14)
$22.6(3)$

40.4(5)

-139.0(4)

159.2(4)

$-20.2(4)$

$-35.6(4)$

$75.0(4)$

$-153.3(3)$

$-142.5(5)$

116.8(6)

65.1(5)

1.0(3)

$-178.4(3)$

$-179.88(18)$

$0.7(4)$

$-1.1(2)$

$-171.6(3)$

179.77(17)

9.3(4)

$-106.9(5)$

$-66.4(3)$

$-84.9(2)$

$-103.2(6)$

96.6(2)

$72.1(5)$

112.5(3)

94.0(2)

$75.8(6)$

$-84.5(2)$

158.7(3)

$-46.2(5)$

$-26.1(3)$

$-28.1(6)$

$-155.1(5)$

46.2(5)

20.1(5)

18.1(7)

$-26.9(5)$

52.1(4)

S-50 
$\mathrm{C}\left(13^{\prime}\right)-\mathrm{C}(12)-\mathrm{C}(13)-\mathrm{Ni}(1)$

$\mathrm{C}(7)-\mathrm{Ni}(1)-\mathrm{C}(13)-\mathrm{C}(14)$

$\mathrm{C}\left(13^{\prime}\right)-\mathrm{Ni}(1)-\mathrm{C}(13)-\mathrm{C}(14)$

$\mathrm{C}(12)-\mathrm{Ni}(1)-\mathrm{C}(13)-\mathrm{C}(14)$

$\mathrm{Cl}(1)-\mathrm{Ni}(1)-\mathrm{C}(13)-\mathrm{C}(14)$

C(7)-Ni(1)-C(13)-C(12)

$\mathrm{C}\left(13^{\prime}\right)-\mathrm{Ni}(1)-\mathrm{C}(13)-\mathrm{C}(12)$

$\mathrm{C}(14)-\mathrm{Ni}(1)-\mathrm{C}(13)-\mathrm{C}(12)$

$\mathrm{Cl}(1)-\mathrm{Ni}(1)-\mathrm{C}(13)-\mathrm{C}(12)$

$\mathrm{C}(12)-\mathrm{C}(13)-\mathrm{C}(14)-\mathrm{C}\left(13^{\prime}\right)$

$\mathrm{Ni}(1)-\mathrm{C}(13)-\mathrm{C}(14)-\mathrm{C}\left(13^{\prime}\right)$

$\mathrm{C}(12)-\mathrm{C}(13)-\mathrm{C}(14)-\mathrm{Ni}(1)$

$\mathrm{C}(7)-\mathrm{Ni}(1)-\mathrm{C}(14)-\mathrm{C}\left(13^{\prime}\right)$

$\mathrm{C}(13)-\mathrm{Ni}(1)-\mathrm{C}(14)-\mathrm{C}\left(13^{\prime}\right)$

C(12)-Ni(1)-C(14)-C(13')

$\mathrm{Cl}(1)-\mathrm{Ni}(1)-\mathrm{C}(14)-\mathrm{C}\left(13^{\prime}\right)$

$\mathrm{C}(7)-\mathrm{Ni}(1)-\mathrm{C}(14)-\mathrm{C}(13)$

$\mathrm{C}\left(13^{\prime}\right)-\mathrm{Ni}(1)-\mathrm{C}(14)-\mathrm{C}(13)$

$\mathrm{C}(12)-\mathrm{Ni}(1)-\mathrm{C}(14)-\mathrm{C}(13)$

$\mathrm{Cl}(1)-\mathrm{Ni}(1)-\mathrm{C}(14)-\mathrm{C}(13)$

$\mathrm{C}(4)-\mathrm{O}(2)-\mathrm{C}\left(5^{\prime}\right)-\mathrm{C}(6)$

$\mathrm{C}(5)-\mathrm{O}(2)-\mathrm{C}\left(5^{\prime}\right)-\mathrm{C}(6)$

$\mathrm{N}(2)-\mathrm{C}(6)-\mathrm{C}\left(5^{\prime}\right)-\mathrm{O}(2)$

$\mathrm{C}(11)-\mathrm{C}(6)-\mathrm{C}\left(5^{\prime}\right)-\mathrm{O}(2)$

$\mathrm{C}(10)-\mathrm{C}(6)-\mathrm{C}\left(5^{\prime}\right)-\mathrm{O}(2)$

$\mathrm{C}\left(10^{\prime}\right)-\mathrm{C}(6)-\mathrm{C}\left(5^{\prime}\right)-\mathrm{O}(2)$

$\mathrm{C}(5)-\mathrm{C}(6)-\mathrm{C}\left(5^{\prime}\right)-\mathrm{O}(2)$

$\mathrm{C}\left(11^{\prime}\right)-\mathrm{C}(6)-\mathrm{C}\left(5^{\prime}\right)-\mathrm{O}(2)$

$\mathrm{C}(13)-\mathrm{C}(14)-\mathrm{C}\left(13^{\prime}\right)-\mathrm{C}(12)$

$\mathrm{Ni}(1)-\mathrm{C}(14)-\mathrm{C}\left(13^{\prime}\right)-\mathrm{C}(12)$

$\mathrm{C}(13)-\mathrm{C}(14)-\mathrm{C}\left(13^{\prime}\right)-\mathrm{Ni}(1)$

$\mathrm{C}(13)-\mathrm{C}(12)-\mathrm{C}\left(13^{\prime}\right)-\mathrm{C}(14)$

$\mathrm{Ni}(1)-\mathrm{C}(12)-\mathrm{C}\left(13^{\prime}\right)-\mathrm{C}(14)$

$\mathrm{C}(13)-\mathrm{C}(12)-\mathrm{C}\left(13^{\prime}\right)-\mathrm{Ni}(1)$

$\mathrm{C}(7)-\mathrm{Ni}(1)-\mathrm{C}\left(13^{\prime}\right)-\mathrm{C}(14)$

$\mathrm{C}(13)-\mathrm{Ni}(1)-\mathrm{C}\left(13^{\prime}\right)-\mathrm{C}(14)$

$\mathrm{C}(12)-\mathrm{Ni}(1)-\mathrm{C}\left(13^{\prime}\right)-\mathrm{C}(14)$

$\mathrm{Cl}(1)-\mathrm{Ni}(1)-\mathrm{C}\left(13^{\prime}\right)-\mathrm{C}(14)$

C(7)-Ni(1)-C(13')-C(12)
$-79.0(5)$

$-165.7(2)$

$-62.7(5)$

$-136.8(5)$

35.7(4)

$-28.9(4)$

74.1(5)

136.8(5)

172.48(15)

29.4(6)

$80.0(5)$

$-50.5(4)$

$-4.9(10)$

$-52.2(6)$

$-24.1(7)$

155.5(7)

47.3(7)

52.2(6)

28.1(3)

$-152.3(3)$

$-32.8(4)$

$58.0(4)$

$30.8(4)$

134.0(4)

$-121.4(5)$

$-84.0(5)$

$-55.7(4)$

154.7(4)

$-32.7(8)$

47.3(8)

$-80.0(4)$

$33.8(8)$

$-49.4(9)$

$83.2(5)$

178.3(3)

74.1(6)

$144.5(10)$

$-30.6(8)$

$33.8(7)$

S-51 
$\mathrm{C}(13)-\mathrm{Ni}(1)-\mathrm{C}\left(13^{\prime}\right)-\mathrm{C}(12)$

$\mathrm{C}(14)-\mathrm{Ni}(1)-\mathrm{C}\left(13^{\prime}\right)-\mathrm{C}(12)$

$\mathrm{Cl}(1)-\mathrm{Ni}(1)-\mathrm{C}\left(13^{\prime}\right)-\mathrm{C}(12)$

C(17D)-C(16)-C(17)-C(18)

$\mathrm{C}(15)-\mathrm{C}(16)-\mathrm{C}(17)-\mathrm{C}(18)$

C(16)-C(17)-C(18)-C(15D)

C(17)-C(18)-C(15D)-C(19)

$\mathrm{C}(18)-\mathrm{C}(15 \mathrm{D})-\mathrm{C}(19)-\mathrm{C}(17 \mathrm{D})$

C(15D)-C(19)-C(17D)-C(16)

C(17)-C(16)-C(17D)-C(19)

C(15)-C(16)-C(17D)-C(19)
$-70.4(6)$

$-144.5(10)$

$-175.16(19)$

0.0

$-178.8(4)$

0.0

0.0

0.0

0.0

0.0

$178.8(5)$

Symmetry transformations used to generate equivalent atoms: 
Table S-13. Crystal data and structure refinement for allyl(MeItBu)NiI (11).

Identification code

Empirical formula

Formula weight

Temperature

Wavelength

Crystal system

Space group

Unit cell dimensions

Volume

Z

Density (calculated)

Absorption coefficient

$\mathrm{F}(000)$

Crystal size

Theta range for data collection

Index ranges

Reflections collected

Independent reflections

Completeness to theta $=27.50^{\circ}$

Absorption correction

Max. and min. transmission

Refinement method

Data / restraints / parameters

Goodness-of-fit on F2

Final $R$ indices $[\mathrm{I}>2 \operatorname{sigma}(\mathrm{I})]$

$\mathrm{R}$ indices (all data)

Largest diff. peak and hole mss031

$\mathrm{C}_{11} \mathrm{H}_{19} \mathrm{IN}_{2} \mathrm{Ni}$

364.89

150(1) K

$0.71073 \AA$

Monoclinic

$\boldsymbol{P} 2{ }_{1} / C$

$\mathrm{a}=8.9469(3) \AA \quad \alpha=90^{\circ}$.

$\mathrm{b}=11.6836(3) \AA \quad \beta=107.2586(13)^{\circ}$.

$\mathrm{c}=13.5112(4) \AA \quad \gamma=90^{\circ}$.

$1348.76(7) \AA^{3}$

4

$1.797 \mathrm{Mg} / \mathrm{m}^{3}$

$3.700 \mathrm{~mm}^{-1}$

720

$0.28 \times 0.20 \times 0.05 \mathrm{~mm}^{3}$

3.00 to $27.50^{\circ}$.

$-11<=\mathrm{h}<=11,-12<=\mathrm{k}<=15,-17<=\mathrm{l}<=17$

5270

$3074[\mathrm{R}(\mathrm{int})=0.0276]$

$99.2 \%$

Multi-scan

0.8366 and 0.4239

Full-matrix least-squares on F2

3074 / 0 / 150

1.058

$\mathrm{R} 1=0.0375, \mathrm{wR} 2=0.0957$

$\mathrm{R} 1=0.0467, \mathrm{wR} 2=0.1017$

1.196 and -1.465 e. $\AA^{-3}$ 
Table S-14. Atomic coordinates ( $\left.\times 10^{4}\right)$ and equivalent isotropic displacement parameters $\left(\AA^{2} \times 10^{3}\right)$ for allyl(MeItBu)NiI (1l). U(eq) is defined as one third of the trace of the orthogonalized $\mathrm{U}^{\mathrm{ij}}$ tensor.

\begin{tabular}{lcccc}
\hline & $\mathrm{x}$ & $\mathrm{y}$ & $\mathrm{z}$ & $\mathrm{U}(\mathrm{eq})$ \\
\hline $\mathrm{Ni}(1)$ & & & & \\
$\mathrm{I}(1)$ & $4223(1)$ & $1319(1)$ & $2137(1)$ & $22(1)$ \\
$\mathrm{N}(1)$ & $6573(1)$ & $2046(1)$ & $3541(1)$ & $29(1)$ \\
$\mathrm{N}(2)$ & $2976(4)$ & $385(3)$ & $3698(2)$ & $20(1)$ \\
$\mathrm{C}(1)$ & $1744(4)$ & $1953(3)$ & $3133(3)$ & $21(1)$ \\
$\mathrm{C}(2)$ & $4014(5)$ & $-604(3)$ & $3799(3)$ & $26(1)$ \\
$\mathrm{C}(3)$ & $1943(5)$ & $555(4)$ & $4264(3)$ & $24(1)$ \\
$\mathrm{C}(4)$ & $1153(5)$ & $1527(4)$ & $3909(3)$ & $26(1)$ \\
$\mathrm{C}(5)$ & $2878(5)$ & $1242(3)$ & $2998(3)$ & $21(1)$ \\
$\mathrm{C}(6)$ & $1068(5)$ & $2962(3)$ & $2476(3)$ & $25(1)$ \\
$\mathrm{C}(7)$ & $465(6)$ & $3829(4)$ & $3111(4)$ & $33(1)$ \\
$\mathrm{C}(8)$ & $-282(6)$ & $2527(4)$ & $1567(4)$ & $41(1)$ \\
$\mathrm{C}(9)$ & $2321(6)$ & $3541(4)$ & $2094(4)$ & $36(1)$ \\
$\mathrm{C}(10)$ & $5379(7)$ & $1178(4)$ & $1025(4)$ & $43(1)$ \\
$\mathrm{C}(10 \mathrm{~A})$ & $3891(9)$ & $1242(7)$ & $604(5)$ & $31(2)$ \\
$\mathrm{C}(11)$ & $4115(17)$ & $363(12)$ & $899(10)$ & $38(4)$ \\
& $2763(7)$ & $535(4)$ & $920(3)$ & $40(1)$ \\
\hline
\end{tabular}


Table S-15. Bond lengths $[\AA]$ and angles $\left[^{\circ}\right]$ for allyl(MeItBu)NiI (1l).

\begin{tabular}{|c|c|}
\hline $\mathrm{Ni}(1)-\mathrm{C}(4)$ & $1.909(4)$ \\
\hline Ni(1)-C(10A) & $1.989(12)$ \\
\hline Ni(1)-C(11) & $1.994(5)$ \\
\hline Ni(1)-C(10) & $2.005(6)$ \\
\hline Ni(1)-C(9) & $2.067(5)$ \\
\hline Ni(1)-I(1) & $2.5241(6)$ \\
\hline $\mathrm{N}(1)-\mathrm{C}(4)$ & $1.362(5)$ \\
\hline $\mathrm{N}(1)-\mathrm{C}(2)$ & $1.378(5)$ \\
\hline $\mathrm{N}(1)-\mathrm{C}(1)$ & $1.463(5)$ \\
\hline $\mathrm{N}(2)-\mathrm{C}(4)$ & $1.365(5)$ \\
\hline $\mathrm{N}(2)-\mathrm{C}(3)$ & $1.397(6)$ \\
\hline $\mathrm{N}(2)-\mathrm{C}(5)$ & $1.491(5)$ \\
\hline $\mathrm{C}(1)-\mathrm{H}(1 \mathrm{~A})$ & 0.9800 \\
\hline $\mathrm{C}(1)-\mathrm{H}(1 \mathrm{~B})$ & 0.9800 \\
\hline $\mathrm{C}(1)-\mathrm{H}(1 \mathrm{C})$ & 0.9800 \\
\hline$C(2)-C(3)$ & $1.348(6)$ \\
\hline $\mathrm{C}(2)-\mathrm{H}(2)$ & 0.9500 \\
\hline $\mathrm{C}(3)-\mathrm{H}(3)$ & 0.9500 \\
\hline$C(5)-C(8)$ & $1.525(6)$ \\
\hline$C(5)-C(6)$ & $1.525(6)$ \\
\hline$C(5)-C(7)$ & $1.533(6)$ \\
\hline $\mathrm{C}(6)-\mathrm{H}(6 \mathrm{~A})$ & 0.9800 \\
\hline $\mathrm{C}(6)-\mathrm{H}(6 \mathrm{~B})$ & 0.9800 \\
\hline $\mathrm{C}(6)-\mathrm{H}(6 \mathrm{C})$ & 0.9800 \\
\hline $\mathrm{C}(7)-\mathrm{H}(7 \mathrm{~A})$ & 0.9800 \\
\hline $\mathrm{C}(7)-\mathrm{H}(7 \mathrm{~B})$ & 0.9800 \\
\hline $\mathrm{C}(7)-\mathrm{H}(7 \mathrm{C})$ & 0.9800 \\
\hline $\mathrm{C}(8)-\mathrm{H}(8 \mathrm{~A})$ & 0.9800 \\
\hline $\mathrm{C}(8)-\mathrm{H}(8 \mathrm{~B})$ & 0.9800 \\
\hline $\mathrm{C}(8)-\mathrm{H}(8 \mathrm{C})$ & 0.9800 \\
\hline $\mathrm{C}(9)-\mathrm{C}(10)$ & $1.286(9)$ \\
\hline$C(9)-C(10 A)$ & $1.449(15)$ \\
\hline $\mathrm{C}(9)-\mathrm{H}(9 \mathrm{~A})$ & 0.9900 \\
\hline $\mathrm{C}(9)-\mathrm{H}(9 \mathrm{~B})$ & 0.9900 \\
\hline $\mathrm{C}(9)-\mathrm{H}(9 \mathrm{C})$ & 0.9900 \\
\hline $\mathrm{C}(9)-\mathrm{H}(9 \mathrm{D})$ & 0.9900 \\
\hline $\mathrm{C}(10)-\mathrm{C}(11)$ & $1.462(9)$ \\
\hline
\end{tabular}




\begin{tabular}{|c|c|}
\hline $\mathrm{C}(10)-\mathrm{H}(10)$ & 0.9500 \\
\hline$C(10 A)-C(11)$ & $1.236(15)$ \\
\hline C(10A)-H(10A) & 0.9500 \\
\hline $\mathrm{C}(11)-\mathrm{H}(11 \mathrm{~A})$ & 0.9900 \\
\hline $\mathrm{C}(11)-\mathrm{H}(11 \mathrm{~B})$ & 0.9900 \\
\hline $\mathrm{C}(11)-\mathrm{H}(11 \mathrm{C})$ & 0.9900 \\
\hline $\mathrm{C}(11)-\mathrm{H}(11 \mathrm{D})$ & 0.9900 \\
\hline $\mathrm{C}(4)-\mathrm{Ni}(1)-\mathrm{C}(10 \mathrm{~A})$ & $127.2(4)$ \\
\hline $\mathrm{C}(4)-\mathrm{Ni}(1)-\mathrm{C}(11)$ & $95.95(19)$ \\
\hline$C(10 A)-N i(1)-C(11)$ & $36.1(4)$ \\
\hline C(4)-Ni(1)-C(10) & $134.5(3)$ \\
\hline$C(10 A)-N i(1)-C(10)$ & $31.9(5)$ \\
\hline C(11)-Ni(1)-C(10) & $42.9(3)$ \\
\hline $\mathrm{C}(4)-\mathrm{Ni}(1)-\mathrm{C}(9)$ & $168.86(19)$ \\
\hline $\mathrm{C}(10 \mathrm{~A})-\mathrm{Ni}(1)-\mathrm{C}(9)$ & $41.8(4)$ \\
\hline $\mathrm{C}(11)-\mathrm{Ni}(1)-\mathrm{C}(9)$ & $73.2(2)$ \\
\hline $\mathrm{C}(10)-\mathrm{Ni}(1)-\mathrm{C}(9)$ & $36.8(3)$ \\
\hline $\mathrm{C}(4)-\mathrm{Ni}(1)-\mathrm{I}(1)$ & $95.31(11)$ \\
\hline $\mathrm{C}(10 \mathrm{~A})-\mathrm{Ni}(1)-\mathrm{I}(1)$ & $130.0(4)$ \\
\hline $\mathrm{C}(11)-\mathrm{Ni}(1)-\mathrm{I}(1)$ & $165.98(17)$ \\
\hline $\mathrm{C}(10)-\mathrm{Ni}(1)-\mathrm{I}(1)$ & $128.8(2)$ \\
\hline C(9)-Ni(1)-I(1) & $95.07(16)$ \\
\hline $\mathrm{C}(4)-\mathrm{N}(1)-\mathrm{C}(2)$ & $111.3(3)$ \\
\hline $\mathrm{C}(4)-\mathrm{N}(1)-\mathrm{C}(1)$ & $123.6(3)$ \\
\hline $\mathrm{C}(2)-\mathrm{N}(1)-\mathrm{C}(1)$ & $125.1(3)$ \\
\hline $\mathrm{C}(4)-\mathrm{N}(2)-\mathrm{C}(3)$ & $110.1(3)$ \\
\hline $\mathrm{C}(4)-\mathrm{N}(2)-\mathrm{C}(5)$ & $126.0(3)$ \\
\hline $\mathrm{C}(3)-\mathrm{N}(2)-\mathrm{C}(5)$ & $123.5(4)$ \\
\hline $\mathrm{N}(1)-\mathrm{C}(1)-\mathrm{H}(1 \mathrm{~A})$ & 109.5 \\
\hline N(1)-C(1)-H(1B) & 109.5 \\
\hline $\mathrm{H}(1 \mathrm{~A})-\mathrm{C}(1)-\mathrm{H}(1 \mathrm{~B})$ & 109.5 \\
\hline $\mathrm{N}(1)-\mathrm{C}(1)-\mathrm{H}(1 \mathrm{C})$ & 109.5 \\
\hline $\mathrm{H}(1 \mathrm{~A})-\mathrm{C}(1)-\mathrm{H}(1 \mathrm{C})$ & 109.5 \\
\hline $\mathrm{H}(1 \mathrm{~B})-\mathrm{C}(1)-\mathrm{H}(1 \mathrm{C})$ & 109.5 \\
\hline $\mathrm{C}(3)-\mathrm{C}(2)-\mathrm{N}(1)$ & $106.9(4)$ \\
\hline $\mathrm{C}(3)-\mathrm{C}(2)-\mathrm{H}(2)$ & 126.5 \\
\hline $\mathrm{N}(1)-\mathrm{C}(2)-\mathrm{H}(2)$ & 126.5 \\
\hline $\mathrm{C}(2)-\mathrm{C}(3)-\mathrm{N}(2)$ & $107.0(4)$ \\
\hline
\end{tabular}




\begin{tabular}{|c|c|}
\hline $\mathrm{C}(2)-\mathrm{C}(3)-\mathrm{H}(3)$ & 126.5 \\
\hline $\mathrm{N}(2)-\mathrm{C}(3)-\mathrm{H}(3)$ & 126.5 \\
\hline $\mathrm{N}(1)-\mathrm{C}(4)-\mathrm{N}(2)$ & $104.6(3)$ \\
\hline $\mathrm{N}(1)-\mathrm{C}(4)-\mathrm{Ni}(1)$ & $122.4(3)$ \\
\hline $\mathrm{N}(2)-\mathrm{C}(4)-\mathrm{Ni}(1)$ & $132.9(3)$ \\
\hline $\mathrm{N}(2)-\mathrm{C}(5)-\mathrm{C}(8)$ & $110.1(3)$ \\
\hline $\mathrm{N}(2)-\mathrm{C}(5)-\mathrm{C}(6)$ & $109.8(3)$ \\
\hline$C(8)-C(5)-C(6)$ & $108.4(4)$ \\
\hline $\mathrm{N}(2)-\mathrm{C}(5)-\mathrm{C}(7)$ & $107.4(3)$ \\
\hline$C(8)-C(5)-C(7)$ & $111.0(4)$ \\
\hline$C(6)-C(5)-C(7)$ & $110.2(4)$ \\
\hline $\mathrm{C}(5)-\mathrm{C}(6)-\mathrm{H}(6 \mathrm{~A})$ & 109.5 \\
\hline $\mathrm{C}(5)-\mathrm{C}(6)-\mathrm{H}(6 \mathrm{~B})$ & 109.5 \\
\hline$H(6 A)-C(6)-H(6 B)$ & 109.5 \\
\hline $\mathrm{C}(5)-\mathrm{C}(6)-\mathrm{H}(6 \mathrm{C})$ & 109.5 \\
\hline $\mathrm{H}(6 \mathrm{~A})-\mathrm{C}(6)-\mathrm{H}(6 \mathrm{C})$ & 109.5 \\
\hline $\mathrm{H}(6 \mathrm{~B})-\mathrm{C}(6)-\mathrm{H}(6 \mathrm{C})$ & 109.5 \\
\hline $\mathrm{C}(5)-\mathrm{C}(7)-\mathrm{H}(7 \mathrm{~A})$ & 109.5 \\
\hline $\mathrm{C}(5)-\mathrm{C}(7)-\mathrm{H}(7 \mathrm{~B})$ & 109.5 \\
\hline $\mathrm{H}(7 \mathrm{~A})-\mathrm{C}(7)-\mathrm{H}(7 \mathrm{~B})$ & 109.5 \\
\hline $\mathrm{C}(5)-\mathrm{C}(7)-\mathrm{H}(7 \mathrm{C})$ & 109.5 \\
\hline $\mathrm{H}(7 \mathrm{~A})-\mathrm{C}(7)-\mathrm{H}(7 \mathrm{C})$ & 109.5 \\
\hline $\mathrm{H}(7 \mathrm{~B})-\mathrm{C}(7)-\mathrm{H}(7 \mathrm{C})$ & 109.5 \\
\hline $\mathrm{C}(5)-\mathrm{C}(8)-\mathrm{H}(8 \mathrm{~A})$ & 109.5 \\
\hline $\mathrm{C}(5)-\mathrm{C}(8)-\mathrm{H}(8 \mathrm{~B})$ & 109.5 \\
\hline $\mathrm{H}(8 \mathrm{~A})-\mathrm{C}(8)-\mathrm{H}(8 \mathrm{~B})$ & 109.5 \\
\hline $\mathrm{C}(5)-\mathrm{C}(8)-\mathrm{H}(8 \mathrm{C})$ & 109.5 \\
\hline $\mathrm{H}(8 \mathrm{~A})-\mathrm{C}(8)-\mathrm{H}(8 \mathrm{C})$ & 109.5 \\
\hline $\mathrm{H}(8 \mathrm{~B})-\mathrm{C}(8)-\mathrm{H}(8 \mathrm{C})$ & 109.5 \\
\hline$C(10)-C(9)-C(10 A)$ & $46.9(7)$ \\
\hline $\mathrm{C}(10)-\mathrm{C}(9)-\mathrm{Ni}(1)$ & $69.0(4)$ \\
\hline $\mathrm{C}(10 \mathrm{~A})-\mathrm{C}(9)-\mathrm{Ni}(1)$ & $66.2(5)$ \\
\hline $\mathrm{C}(10)-\mathrm{C}(9)-\mathrm{H}(9 \mathrm{~A})$ & 116.8 \\
\hline $\mathrm{C}(10 \mathrm{~A})-\mathrm{C}(9)-\mathrm{H}(9 \mathrm{~A})$ & 162.7 \\
\hline $\mathrm{Ni}(1)-\mathrm{C}(9)-\mathrm{H}(9 \mathrm{~A})$ & 116.8 \\
\hline $\mathrm{C}(10)-\mathrm{C}(9)-\mathrm{H}(9 \mathrm{~B})$ & 116.8 \\
\hline $\mathrm{C}(10 \mathrm{~A})-\mathrm{C}(9)-\mathrm{H}(9 \mathrm{~B})$ & 76.4 \\
\hline $\mathrm{Ni}(1)-\mathrm{C}(9)-\mathrm{H}(9 \mathrm{~B})$ & 116.8 \\
\hline $\mathrm{H}(9 \mathrm{~A})-\mathrm{C}(9)-\mathrm{H}(9 \mathrm{~B})$ & 113.8 \\
\hline
\end{tabular}




\begin{tabular}{|c|c|}
\hline C(10)-C(9)-H(9C) & 74.4 \\
\hline $\mathrm{C}(10 \mathrm{~A})-\mathrm{C}(9)-\mathrm{H}(9 \mathrm{C})$ & 117.1 \\
\hline Ni(1)-C(9)-H(9C) & 117.1 \\
\hline $\mathrm{H}(9 \mathrm{~A})-\mathrm{C}(9)-\mathrm{H}(9 \mathrm{C})$ & 45.7 \\
\hline $\mathrm{H}(9 \mathrm{~B})-\mathrm{C}(9)-\mathrm{H}(9 \mathrm{C})$ & 125.1 \\
\hline $\mathrm{C}(10)-\mathrm{C}(9)-\mathrm{H}(9 \mathrm{D})$ & 161.1 \\
\hline C(10A)-C(9)-H(9D) & 117.1 \\
\hline Ni(1)-C(9)-H(9D) & 117.1 \\
\hline $\mathrm{H}(9 \mathrm{~A})-\mathrm{C}(9)-\mathrm{H}(9 \mathrm{D})$ & 77.6 \\
\hline H(9B)-C(9)-H(9D) & 44.4 \\
\hline $\mathrm{H}(9 \mathrm{C})-\mathrm{C}(9)-\mathrm{H}(9 \mathrm{D})$ & 114.1 \\
\hline $\mathrm{C}(9)-\mathrm{C}(10)-\mathrm{C}(11)$ & $123.5(6)$ \\
\hline $\mathrm{C}(9)-\mathrm{C}(10)-\mathrm{Ni}(1)$ & $74.2(4)$ \\
\hline $\mathrm{C}(11)-\mathrm{C}(10)-\mathrm{Ni}(1)$ & $68.2(3)$ \\
\hline C(9)-C(10)-H(10) & 118.2 \\
\hline $\mathrm{C}(11)-\mathrm{C}(10)-\mathrm{H}(10)$ & 118.2 \\
\hline Ni(1)-C(10)-H(10) & 132.8 \\
\hline $\mathrm{C}(11)-\mathrm{C}(10 \mathrm{~A})-\mathrm{C}(9)$ & $128.7(11)$ \\
\hline $\mathrm{C}(11)-\mathrm{C}(10 \mathrm{~A})-\mathrm{Ni}(1)$ & $72.1(6)$ \\
\hline C(9)-C(10A)-Ni(1) & $72.0(6)$ \\
\hline $\mathrm{C}(11)-\mathrm{C}(10 \mathrm{~A})-\mathrm{H}(10 \mathrm{~A})$ & 115.6 \\
\hline $\mathrm{C}(9)-\mathrm{C}(10 \mathrm{~A})-\mathrm{H}(10 \mathrm{~A})$ & 115.6 \\
\hline $\mathrm{Ni}(1)-\mathrm{C}(10 \mathrm{~A})-\mathrm{H}(10 \mathrm{~A})$ & 135.5 \\
\hline$C(10 A)-C(11)-C(10)$ & $47.1(7)$ \\
\hline C(10A)-C(11)-Ni(1) & $71.7(6)$ \\
\hline $\mathrm{C}(10)-\mathrm{C}(11)-\mathrm{Ni}(1)$ & $69.0(3)$ \\
\hline $\mathrm{C}(10 \mathrm{~A})-\mathrm{C}(11)-\mathrm{H}(11 \mathrm{~A})$ & 73.7 \\
\hline $\mathrm{C}(10)-\mathrm{C}(11)-\mathrm{H}(11 \mathrm{~A})$ & 116.8 \\
\hline Ni(1)-C(11)-H(11A) & 116.8 \\
\hline $\mathrm{C}(10 \mathrm{~A})-\mathrm{C}(11)-\mathrm{H}(11 \mathrm{~B})$ & 160.0 \\
\hline $\mathrm{C}(10)-\mathrm{C}(11)-\mathrm{H}(11 \mathrm{~B})$ & 116.8 \\
\hline $\mathrm{Ni}(1)-\mathrm{C}(11)-\mathrm{H}(11 \mathrm{~B})$ & 116.8 \\
\hline $\mathrm{H}(11 \mathrm{~A})-\mathrm{C}(11)-\mathrm{H}(11 \mathrm{~B})$ & 113.8 \\
\hline $\mathrm{C}(10 \mathrm{~A})-\mathrm{C}(11)-\mathrm{H}(11 \mathrm{C})$ & 116.4 \\
\hline $\mathrm{C}(10)-\mathrm{C}(11)-\mathrm{H}(11 \mathrm{C})$ & 162.0 \\
\hline Ni(1)-C(11)-H(11C) & 116.4 \\
\hline $\mathrm{H}(11 \mathrm{~A})-\mathrm{C}(11)-\mathrm{H}(11 \mathrm{C})$ & 45.2 \\
\hline $\mathrm{H}(11 \mathrm{~B})-\mathrm{C}(11)-\mathrm{H}(11 \mathrm{C})$ & 77.2 \\
\hline $\mathrm{C}(10 \mathrm{~A})-\mathrm{C}(11)-\mathrm{H}(11 \mathrm{D})$ & 116.4 \\
\hline
\end{tabular}


C(10)-C(11)-H(11D) $\quad 75.7$

Ni(1)-C(11)-H(11D) 116.4

H(11A)-C(11)-H(11D) 126.1

H(11B)-C(11)-H(11D) 43.9

H(11C)-C(11)-H(11D) 113.4

Symmetry transformations used to generate equivalent atoms: 
Table S-16. Anisotropic displacement parameters $\left(\AA^{2} \times 10^{3}\right)$ for allyl(MeItBu)NiI (11). The anisotropic displacement factor exponent takes the form: $-2 \pi^{2}\left[h^{2} a^{* 2} U^{11}+\ldots+2 h k a^{*} b^{*} U^{12}\right]$

\begin{tabular}{lcccccc}
\hline & $\mathrm{U}^{11}$ & $\mathrm{U}^{22}$ & $\mathrm{U}^{33}$ & $\mathrm{U}^{23}$ & $\mathrm{U}^{13}$ & $\mathrm{U}^{12}$ \\
\hline $\mathrm{Ni}(1)$ & $28(1)$ & $23(1)$ & $17(1)$ & $0(1)$ & $6(1)$ & $3(1)$ \\
$\mathrm{I}(1)$ & $29(1)$ & $30(1)$ & $27(1)$ & $4(1)$ & $7(1)$ & $-1(1)$ \\
$\mathrm{N}(1)$ & $23(2)$ & $19(2)$ & $17(2)$ & $-1(1)$ & $2(1)$ & $0(1)$ \\
$\mathrm{N}(2)$ & $21(2)$ & $21(2)$ & $17(2)$ & $2(1)$ & $1(1)$ & $2(1)$ \\
$\mathrm{C}(1)$ & $33(2)$ & $20(2)$ & $24(2)$ & $2(2)$ & $6(2)$ & $3(2)$ \\
$\mathrm{C}(2)$ & $26(2)$ & $26(2)$ & $21(2)$ & $1(2)$ & $7(2)$ & $-6(2)$ \\
$\mathrm{C}(3)$ & $25(2)$ & $31(2)$ & $24(2)$ & $2(2)$ & $8(2)$ & $1(2)$ \\
$\mathrm{C}(4)$ & $22(2)$ & $21(2)$ & $17(2)$ & $-4(2)$ & $1(2)$ & $-2(2)$ \\
$\mathrm{C}(5)$ & $26(2)$ & $24(2)$ & $21(2)$ & $4(2)$ & $1(2)$ & $7(2)$ \\
$\mathrm{C}(6)$ & $38(3)$ & $30(2)$ & $29(2)$ & $2(2)$ & $6(2)$ & $11(2)$ \\
$\mathrm{C}(7)$ & $40(3)$ & $37(3)$ & $34(3)$ & $-2(2)$ & $-9(2)$ & $7(2)$ \\
$\mathrm{C}(8)$ & $46(3)$ & $25(2)$ & $38(3)$ & $9(2)$ & $17(2)$ & $8(2)$ \\
$\mathrm{C}(9)$ & $72(4)$ & $37(3)$ & $31(2)$ & $-9(2)$ & $32(3)$ & $-13(3)$ \\
$\mathrm{C}(10)$ & $45(5)$ & $35(5)$ & $11(3)$ & $-3(3)$ & $8(3)$ & $5(3)$ \\
$\mathrm{C}(10 \mathrm{~A})$ & $58(9)$ & $39(8)$ & $25(7)$ & $-8(6)$ & $25(6)$ & $1(6)$ \\
$\mathrm{C}(11)$ & $60(3)$ & $38(3)$ & $19(2)$ & $-5(2)$ & $7(2)$ & $-10(2)$ \\
& & & & & & \\
\hline
\end{tabular}


Table S-17. Hydrogen coordinates ( $\left.\times 10^{4}\right)$ and isotropic displacement parameters $\left(\AA^{2} \times 10^{3}\right)$ for allyl(MeItBu)NiI (1l).

\begin{tabular}{|c|c|c|c|c|}
\hline & $\mathrm{x}$ & $\mathrm{y}$ & z & $\mathrm{U}(\mathrm{eq})$ \\
\hline $\mathrm{H}(1 \mathrm{~A})$ & 3618 & -1107 & 3198 & 39 \\
\hline $\mathrm{H}(1 \mathrm{~B})$ & 4051 & -1024 & 4433 & 39 \\
\hline $\mathrm{H}(1 \mathrm{C})$ & 5069 & -341 & 3834 & 39 \\
\hline $\mathrm{H}(2)$ & 1812 & 79 & 4802 & 29 \\
\hline $\mathrm{H}(3)$ & 348 & 1859 & 4141 & 32 \\
\hline $\mathrm{H}(6 \mathrm{~A})$ & 54 & 4501 & 2683 & 49 \\
\hline $\mathrm{H}(6 \mathrm{~B})$ & 1323 & 4062 & 3717 & 49 \\
\hline $\mathrm{H}(6 \mathrm{C})$ & -371 & 3480 & 3342 & 49 \\
\hline $\mathrm{H}(7 \mathrm{~A})$ & 109 & 1945 & 1184 & 62 \\
\hline $\mathrm{H}(7 \mathrm{~B})$ & -727 & 3167 & 1103 & 62 \\
\hline $\mathrm{H}(7 \mathrm{C})$ & -1093 & 2191 & 1831 & 62 \\
\hline $\mathrm{H}(8 \mathrm{~A})$ & 2634 & 3028 & 1617 & 53 \\
\hline $\mathrm{H}(8 \mathrm{~B})$ & 3234 & 3716 & 2686 & 53 \\
\hline $\mathrm{H}(8 \mathrm{C})$ & 1902 & 4252 & 1733 & 53 \\
\hline $\mathrm{H}(9 \mathrm{~A})$ & 6006 & 1846 & 935 & 52 \\
\hline $\mathrm{H}(9 \mathrm{~B})$ & 5878 & 432 & 978 & 52 \\
\hline $\mathrm{H}(9 \mathrm{C})$ & 5218 & 1801 & 508 & 52 \\
\hline $\mathrm{H}(9 \mathrm{D})$ & 6459 & 872 & 1253 & 52 \\
\hline $\mathrm{H}(10)$ & 3503 & 1776 & 58 & 37 \\
\hline $\mathrm{H}(10 \mathrm{~A})$ & 4360 & -408 & 785 & 46 \\
\hline $\mathrm{H}(11 \mathrm{~A})$ & 2876 & -304 & 876 & 48 \\
\hline $\mathrm{H}(11 \mathrm{~B})$ & 1662 & 800 & 704 & 48 \\
\hline $\mathrm{H}(11 \mathrm{C})$ & 2192 & -136 & 1072 & 48 \\
\hline $\mathrm{H}(11 \mathrm{D})$ & 2108 & 1036 & 369 & 48 \\
\hline
\end{tabular}


Table S-18. Torsion angles $\left[^{\circ}\right]$ for allyl(MeItBu)NiI (11).

\begin{tabular}{lc}
\hline $\mathrm{C}(4)-\mathrm{N}(1)-\mathrm{C}(2)-\mathrm{C}(3)$ & $1.0(5)$ \\
$\mathrm{C}(1)-\mathrm{N}(1)-\mathrm{C}(2)-\mathrm{C}(3)$ & $-177.0(4)$ \\
$\mathrm{N}(1)-\mathrm{C}(2)-\mathrm{C}(3)-\mathrm{N}(2)$ & $-1.1(4)$ \\
$\mathrm{C}(4)-\mathrm{N}(2)-\mathrm{C}(3)-\mathrm{C}(2)$ & $0.8(5)$ \\
$\mathrm{C}(5)-\mathrm{N}(2)-\mathrm{C}(3)-\mathrm{C}(2)$ & $174.0(4)$ \\
$\mathrm{C}(2)-\mathrm{N}(1)-\mathrm{C}(4)-\mathrm{N}(2)$ & $-0.5(4)$ \\
$\mathrm{C}(1)-\mathrm{N}(1)-\mathrm{C}(4)-\mathrm{N}(2)$ & $177.6(3)$ \\
$\mathrm{C}(2)-\mathrm{N}(1)-\mathrm{C}(4)-\mathrm{Ni}(1)$ & $176.6(3)$ \\
$\mathrm{C}(1)-\mathrm{N}(1)-\mathrm{C}(4)-\mathrm{Ni}(1)$ & $-5.4(5)$ \\
$\mathrm{C}(3)-\mathrm{N}(2)-\mathrm{C}(4)-\mathrm{N}(1)$ & $-0.2(4)$ \\
$\mathrm{C}(5)-\mathrm{N}(2)-\mathrm{C}(4)-\mathrm{N}(1)$ & $-173.1(3)$ \\
$\mathrm{C}(3)-\mathrm{N}(2)-\mathrm{C}(4)-\mathrm{Ni}(1)$ & $-176.8(3)$ \\
$\mathrm{C}(5)-\mathrm{N}(2)-\mathrm{C}(4)-\mathrm{Ni}(1)$ & $10.3(6)$ \\
$\mathrm{C}(10 \mathrm{~A})-\mathrm{Ni}(1)-\mathrm{C}(4)-\mathrm{N}(1)$ & $74.8(6)$ \\
$\mathrm{C}(11)-\mathrm{Ni}(1)-\mathrm{C}(4)-\mathrm{N}(1)$ & $94.8(3)$ \\
$\mathrm{C}(10)-\mathrm{Ni}(1)-\mathrm{C}(4)-\mathrm{N}(1)$ & $116.4(4)$ \\
$\mathrm{C}(9)-\mathrm{Ni}(1)-\mathrm{C}(4)-\mathrm{N}(1)$ & $81.8(11)$ \\
$\mathrm{I}(1)-\mathrm{Ni}(1)-\mathrm{C}(4)-\mathrm{N}(1)$ & $-76.8(3)$ \\
$\mathrm{C}(10 \mathrm{~A})-\mathrm{Ni}(1)-\mathrm{C}(4)-\mathrm{N}(2)$ & $-109.0(6)$ \\
$\mathrm{C}(11)-\mathrm{Ni}(1)-\mathrm{C}(4)-\mathrm{N}(2)$ & $-89.0(4)$ \\
$\mathrm{C}(10)-\mathrm{Ni}(1)-\mathrm{C}(4)-\mathrm{N}(2)$ & $-67.5(5)$ \\
$\mathrm{C}(9)-\mathrm{Ni}(1)-\mathrm{C}(4)-\mathrm{N}(2)$ & $-102.1(11)$ \\
$\mathrm{I}(1)-\mathrm{Ni}(1)-\mathrm{C}(4)-\mathrm{N}(2)$ & $99.3(4)$ \\
$\mathrm{C}(4)-\mathrm{N}(2)-\mathrm{C}(5)-\mathrm{C}(8)$ & $-34.3(5)$ \\
$\mathrm{C}(3)-\mathrm{N}(2)-\mathrm{C}(5)-\mathrm{C}(8)$ & $153.7(4)$ \\
$\mathrm{C}(4)-\mathrm{N}(2)-\mathrm{C}(5)-\mathrm{C}(6)$ & $-153.5(4)$ \\
$\mathrm{C}(3)-\mathrm{N}(2)-\mathrm{C}(5)-\mathrm{C}(6)$ & $34.5(5)$ \\
$\mathrm{C}(4)-\mathrm{N}(2)-\mathrm{C}(5)-\mathrm{C}(7)$ & $86.7(5)$ \\
$\mathrm{C}(3)-\mathrm{N}(2)-\mathrm{C}(5)-\mathrm{C}(7)$ & $-85.3(5)$ \\
$\mathrm{C}(4)-\mathrm{Ni}(1)-\mathrm{C}(9)-\mathrm{C}(10)$ & $42.5(12)$ \\
$\mathrm{C}(10 \mathrm{~A})-\mathrm{Ni}(1)-\mathrm{C}(9)-\mathrm{C}(10)$ & $50.9(7)$ \\
$\mathrm{C}(11)-\mathrm{Ni}(1)-\mathrm{C}(9)-\mathrm{C}(10)$ & $29.0(4)$ \\
$\mathrm{I}(1)-\mathrm{Ni}(1)-\mathrm{C}(9)-\mathrm{C}(10)$ & $-158.8(4)$ \\
$\mathrm{C}(4)-\mathrm{Ni}(1)-\mathrm{C}(9)-\mathrm{C}(10 \mathrm{~A})$ & $-8.3(14)$ \\
$\mathrm{C}(11)-\mathrm{Ni}(1)-\mathrm{C}(9)-\mathrm{C}(10 \mathrm{~A})$ & $-9(7)$ \\
$\mathrm{C}(10)-\mathrm{Ni}(1)-\mathrm{C}(9)-\mathrm{C}(10 \mathrm{~A})$ & $\mathrm{I})$ \\
$\mathrm{I}(1)-\mathrm{Ni}(1)-\mathrm{C}(9)-\mathrm{C}(10 \mathrm{~A})$ & \\
& \\
& \\
&
\end{tabular}


C(10A)-C(9)-C(10)-C(11)

$\mathrm{Ni}(1)-\mathrm{C}(9)-\mathrm{C}(10)-\mathrm{C}(11)$

$\mathrm{C}(10 \mathrm{~A})-\mathrm{C}(9)-\mathrm{C}(10)-\mathrm{Ni}(1)$

$\mathrm{C}(4)-\mathrm{Ni}(1)-\mathrm{C}(10)-\mathrm{C}(9)$

$\mathrm{C}(10 \mathrm{~A})-\mathrm{Ni}(1)-\mathrm{C}(10)-\mathrm{C}(9)$

$\mathrm{C}(11)-\mathrm{Ni}(1)-\mathrm{C}(10)-\mathrm{C}(9)$

$\mathrm{I}(1)-\mathrm{Ni}(1)-\mathrm{C}(10)-\mathrm{C}(9)$

C(4)-Ni(1)-C(10)-C(11)

C(10A)-Ni(1)-C(10)-C(11)

$\mathrm{C}(9)-\mathrm{Ni}(1)-\mathrm{C}(10)-\mathrm{C}(11)$

$\mathrm{I}(1)-\mathrm{Ni}(1)-\mathrm{C}(10)-\mathrm{C}(11)$

$\mathrm{C}(10)-\mathrm{C}(9)-\mathrm{C}(10 \mathrm{~A})-\mathrm{C}(11)$

$\mathrm{Ni}(1)-\mathrm{C}(9)-\mathrm{C}(10 \mathrm{~A})-\mathrm{C}(11)$

$\mathrm{C}(10)-\mathrm{C}(9)-\mathrm{C}(10 \mathrm{~A})-\mathrm{Ni}(1)$

$\mathrm{C}(4)-\mathrm{Ni}(1)-\mathrm{C}(10 \mathrm{~A})-\mathrm{C}(11)$

C(10)-Ni(1)-C(10A)-C(11)

$\mathrm{C}(9)-\mathrm{Ni}(1)-\mathrm{C}(10 \mathrm{~A})-\mathrm{C}(11)$

$\mathrm{I}(1)-\mathrm{Ni}(1)-\mathrm{C}(10 \mathrm{~A})-\mathrm{C}(11)$

$\mathrm{C}(4)-\mathrm{Ni}(1)-\mathrm{C}(10 \mathrm{~A})-\mathrm{C}(9)$

$\mathrm{C}(11)-\mathrm{Ni}(1)-\mathrm{C}(10 \mathrm{~A})-\mathrm{C}(9)$

$\mathrm{C}(10)-\mathrm{Ni}(1)-\mathrm{C}(10 \mathrm{~A})-\mathrm{C}(9)$

I(1)-Ni(1)-C(10A)-C(9)

$\mathrm{C}(9)-\mathrm{C}(10 \mathrm{~A})-\mathrm{C}(11)-\mathrm{C}(10)$

$\mathrm{Ni}(1)-\mathrm{C}(10 \mathrm{~A})-\mathrm{C}(11)-\mathrm{C}(10)$

$\mathrm{C}(9)-\mathrm{C}(10 \mathrm{~A})-\mathrm{C}(11)-\mathrm{Ni}(1)$

$\mathrm{C}(9)-\mathrm{C}(10)-\mathrm{C}(11)-\mathrm{C}(10 \mathrm{~A})$

$\mathrm{Ni}(1)-\mathrm{C}(10)-\mathrm{C}(11)-\mathrm{C}(10 \mathrm{~A})$

$\mathrm{C}(9)-\mathrm{C}(10)-\mathrm{C}(11)-\mathrm{Ni}(1)$

$\mathrm{C}(4)-\mathrm{Ni}(1)-\mathrm{C}(11)-\mathrm{C}(10 \mathrm{~A})$

C(10)-Ni(1)-C(11)-C(10A)

$\mathrm{C}(9)-\mathrm{Ni}(1)-\mathrm{C}(11)-\mathrm{C}(10 \mathrm{~A})$

I(1)-Ni(1)-C(11)-C(10A)

$\mathrm{C}(4)-\mathrm{Ni}(1)-\mathrm{C}(11)-\mathrm{C}(10)$

$\mathrm{C}(10 \mathrm{~A})-\mathrm{Ni}(1)-\mathrm{C}(11)-\mathrm{C}(10)$

$\mathrm{C}(9)-\mathrm{Ni}(1)-\mathrm{C}(11)-\mathrm{C}(10)$

I(1)-Ni(1)-C(11)-C(10)
27.2(7)

$-49.4(5)$

$76.6(7)$

$-169.4(3)$

$-78.0(9)$

$-137.0(6)$

27.5(5)

$-32.5(5)$

58.9(9)

137.0(6)

164.5(3)

$-35.3(11)$

47.5(11)

$-82.8(6)$

35.2(9)

$-81.3(10)$

$-142.8(11)$

177.1(3)

178.0(3)

142.8(11)

61.5(8)

$-40.1(8)$

30.4(9)

77.9(7)

$-47.5(11)$

$-32.3(9)$

$-84.2(9)$

51.9(6)

$-152.5(7)$

50.2(8)

24.9(7)

$-9.2(11)$

157.4(4)

$-50.2(8)$

$-25.2(4)$

$-59.4(8)$

Symmetry transformations used to generate equivalent atoms: 
Table S-19. Crystal data and structure refinement for trans-[ $\left.\mathrm{ITol}_{2} \mathrm{NiCl}_{2}\right](3 \mathbf{h})$.

Identification code

Empirical formula

Formula weight

Temperature

Wavelength

Crystal system

Space group

Unit cell dimensions

Volume

$\mathrm{Z}$

Density (calculated)

Absorption coefficient

$\mathrm{F}(000)$

Crystal size

Theta range for data collection

Index ranges

Reflections collected

Independent reflections

Completeness to theta $=27.48^{\circ}$

Absorption correction

Max. and min. transmission

Refinement method

Data / restraints / parameters

Goodness-of-fit on F2

Final $\mathrm{R}$ indices [I $>2 \operatorname{sigma}(\mathrm{I})]$

$\mathrm{R}$ indices (all data)

Extinction coefficient

Largest diff. peak and hole mss021

$\mathrm{C}_{34} \mathrm{H}_{32} \mathrm{Cl}_{2} \mathrm{~N}_{4} \mathrm{Ni}$

626.25

150(1) K

$0.71073 \AA$

Triclinic

$\mathrm{P}$

$$
\begin{array}{ll}
\mathrm{a}=10.0381(2) \AA & \alpha=91.5563(12)^{\circ} . \\
\mathrm{b}=12.4276(3) \AA & \beta=111.4776(16)^{\circ} . \\
\mathrm{c}=13.2040(5) \AA & \gamma=102.8770(16)^{\circ} .
\end{array}
$$$$
\text { 1483.48(7) } \AA^{3}
$$

2

$1.402 \mathrm{Mg} / \mathrm{m}^{3}$

$0.865 \mathrm{~mm}^{-1}$

652

$0.33 \times 0.18 \times 0.13 \mathrm{~mm}^{3}$

1.67 to $27.48^{\circ}$.

$-12<=\mathrm{h}<=13,-14<=\mathrm{k}<=16,-15<=\mathrm{l}<=17$

10438

$6647[\mathrm{R}(\mathrm{int})=0.0360]$

$97.8 \%$

Multi-scan

0.8959 and 0.7634

Full-matrix least-squares on F2

6647 / 0 / 375

1.027

$\mathrm{R} 1=0.0449, \mathrm{wR} 2=0.1028$

$\mathrm{R} 1=0.0685, \mathrm{wR} 2=0.1201$

0.0072(9)

0.509 and -0.774 e. $\AA^{-3}$ 
Table S-20. Atomic coordinates ( $\left.\times 10^{4}\right)$ and equivalent isotropic displacement parameters $\left(\AA^{2} \times 10^{3}\right)$ for trans-[$\left[\mathrm{ITol}_{2} \mathrm{NiCl}_{2}\right](3 \mathbf{h}) . \mathrm{U}(\mathrm{eq})$ is defined as one third of the trace of the orthogonalized $\mathrm{U}^{\mathrm{ij}}$ tensor.

\begin{tabular}{|c|c|c|c|c|}
\hline & $\mathrm{x}$ & $\mathrm{y}$ & $\mathrm{z}$ & $\mathrm{U}(\mathrm{eq})$ \\
\hline $\mathrm{Ni}(1)$ & $2387(1)$ & $2317(1)$ & $2513(1)$ & 21(1) \\
\hline $\mathrm{Cl}(1)$ & $3760(1)$ & $3853(1)$ & $2272(1)$ & $30(1)$ \\
\hline $\mathrm{Cl}(2)$ & 1062(1) & 788(1) & 2804(1) & $29(1)$ \\
\hline $\mathrm{N}(1)$ & $3323(2)$ & $1225(2)$ & $963(2)$ & $25(1)$ \\
\hline $\mathrm{N}(2)$ & $1369(2)$ & $1765(2)$ & $113(2)$ & $26(1)$ \\
\hline $\mathrm{N}(3)$ & $3669(2)$ & $3210(2)$ & $4850(2)$ & $24(1)$ \\
\hline $\mathrm{N}(4)$ & $1514(2)$ & $3494(2)$ & $4061(2)$ & $24(1)$ \\
\hline $\mathrm{C}(1)$ & $5924(3)$ & $1277(2)$ & $1917(2)$ & $32(1)$ \\
\hline$C(2)$ & 7014(3) & 784(3) & $2557(3)$ & $37(1)$ \\
\hline$C(3)$ & $6658(3)$ & $-146(3)$ & $3053(2)$ & $33(1)$ \\
\hline $\mathrm{C}(4)$ & $5184(3)$ & $-559(3)$ & 2921(2) & $32(1)$ \\
\hline$C(5)$ & 4083(3) & $-78(2)$ & $2273(2)$ & $30(1)$ \\
\hline$C(6)$ & 4471(3) & $827(2)$ & $1764(2)$ & $27(1)$ \\
\hline$C(7)$ & 7834(4) & $-698(3)$ & $3740(3)$ & $46(1)$ \\
\hline$C(8)$ & $3055(3)$ & 1071(2) & $-150(2)$ & $32(1)$ \\
\hline $\mathrm{C}(9)$ & 1832(3) & $1409(2)$ & $-682(2)$ & $31(1)$ \\
\hline$C(10)$ & 2298(3) & $1676(2)$ & $1144(2)$ & $23(1)$ \\
\hline $\mathrm{C}(11)$ & $39(3)$ & $2137(2)$ & $-165(2)$ & $28(1)$ \\
\hline$C(12)$ & $-1024(3)$ & $1654(2)$ & $233(2)$ & $31(1)$ \\
\hline $\mathrm{C}(13)$ & $-2348(3)$ & 1969(3) & $-110(3)$ & $35(1)$ \\
\hline$C(14)$ & $-2641(3)$ & $2757(2)$ & $-832(2)$ & $34(1)$ \\
\hline$C(15)$ & $-1552(3)$ & $3229(3)$ & $-1211(3)$ & $37(1)$ \\
\hline$C(16)$ & $-219(3)$ & 2925(3) & $-881(3)$ & $37(1)$ \\
\hline$C(17)$ & $-4088(4)$ & $3084(3)$ & $-1211(3)$ & $47(1)$ \\
\hline$C(18)$ & $5049(3)$ & $1847(2)$ & $4735(2)$ & $28(1)$ \\
\hline$C(19)$ & $6387(3)$ & $1554(2)$ & $5039(2)$ & $31(1)$ \\
\hline$C(20)$ & $7721(3)$ & $2306(2)$ & $5663(2)$ & $29(1)$ \\
\hline$C(21)$ & $7679(3)$ & $3369(2)$ & $5980(2)$ & $31(1)$ \\
\hline$C(22)$ & $6350(3)$ & $3671(2)$ & $5698(2)$ & $30(1)$ \\
\hline$C(23)$ & $5039(3)$ & $2905(2)$ & $5081(2)$ & $24(1)$ \\
\hline$C(24)$ & $9157(3)$ & 1963(3) & $5995(3)$ & $38(1)$ \\
\hline$C(25)$ & $3380(3)$ & $3763(2)$ & $5652(2)$ & $29(1)$ \\
\hline$C(26)$ & 2032(3) & $3934(2)$ & $5156(2)$ & $29(1)$ \\
\hline$C(27)$ & $2525(3)$ & $3034(2)$ & $3857(2)$ & $22(1)$ \\
\hline
\end{tabular}




$\begin{array}{lllll}\mathrm{C}(28) & 206(3) & 3706(2) & 3260(2) & 23(1) \\ \mathrm{C}(29) & -1004(3) & 3682(2) & 3541(2) & 30(1) \\ \mathrm{C}(30) & -2179(3) & 4058(2) & 2850(2) & 32(1) \\ \mathrm{C}(31) & -2178(3) & 4459(2) & 1879(2) & 32(1) \\ \mathrm{C}(32) & -987(3) & 4412(2) & 1588(2) & 29(1) \\ \mathrm{C}(33) & 206(3) & 4043(2) & 2270(2) & 26(1) \\ \mathrm{C}(34) & -3392(4) & 4962(3) & 1178(3) & 47(1)\end{array}$


Table S-21. Bond lengths $[\AA]$ and angles $\left[{ }^{\circ}\right]$ for trans- $\left[\mathrm{ITol}_{2} \mathrm{NiCl}_{2}\right](3 \mathbf{h})$.

\begin{tabular}{ll}
\hline $\mathrm{Ni}(1)-\mathrm{C}(27)$ & $1.909(3)$ \\
$\mathrm{Ni}(1)-\mathrm{C}(10)$ & $1.918(3)$ \\
$\mathrm{Ni}(1)-\mathrm{Cl}(2)$ & $2.1940(7)$ \\
$\mathrm{Ni}(1)-\mathrm{Cl}(1)$ & $2.1965(7)$ \\
$\mathrm{N}(1)-\mathrm{C}(10)$ & $1.363(3)$ \\
$\mathrm{N}(1)-\mathrm{C}(8)$ & $1.393(3)$ \\
$\mathrm{N}(1)-\mathrm{C}(6)$ & $1.443(3)$ \\
$\mathrm{N}(2)-\mathrm{C}(10)$ & $1.364(3)$ \\
$\mathrm{N}(2)-\mathrm{C}(9)$ & $1.393(3)$ \\
$\mathrm{N}(2)-\mathrm{C}(11)$ & $1.434(3)$ \\
$\mathrm{N}(3)-\mathrm{C}(27)$ & $1.362(3)$ \\
$\mathrm{N}(3)-\mathrm{C}(25)$ & $1.397(3)$ \\
$\mathrm{N}(3)-\mathrm{C}(23)$ & $1.436(3)$ \\
$\mathrm{N}(4)-\mathrm{C}(27)$ & $1.370(3)$ \\
$\mathrm{N}(4)-\mathrm{C}(26)$ & $1.394(3)$ \\
$\mathrm{N}(4)-\mathrm{C}(28)$ & $1.437(3)$ \\
$\mathrm{C}(1)-\mathrm{C}(6)$ & $1.377(4)$ \\
$\mathrm{C}(1)-\mathrm{C}(2)$ & $1.392(4)$ \\
$\mathrm{C}(1)-\mathrm{H}(1)$ & 0.9500 \\
$\mathrm{C}(2)-\mathrm{C}(3)$ & $1.386(4)$ \\
$\mathrm{C}(2)-\mathrm{H}(2)$ & 0.9500 \\
$\mathrm{C}(3)-\mathrm{C}(4)$ & $1.396(4)$ \\
$\mathrm{C}(3)-\mathrm{C}(7)$ & $1.516(4)$ \\
$\mathrm{C}(4)-\mathrm{C}(5)$ & $1.393(4)$ \\
$\mathrm{C}(4)-\mathrm{H}(4)$ & 0.9500 \\
$\mathrm{C}(5)-\mathrm{C}(6)$ & $1.380(4)$ \\
$\mathrm{C}(5)-\mathrm{H}(5)$ & 0.9500 \\
$\mathrm{C}(7)-\mathrm{H}(7 \mathrm{~A})$ & 0.9800 \\
$\mathrm{C}(7)-\mathrm{H}(7 \mathrm{~B})$ & 0.9800 \\
$\mathrm{C}(7)-\mathrm{H}(7 \mathrm{C})$ & 0.9800 \\
$\mathrm{C}(8)-\mathrm{C}(9)$ & $1.338(4)$ \\
$\mathrm{C}(8)-\mathrm{H}(8)$ & 0.9500 \\
$\mathrm{C}(9)-\mathrm{H}(9)$ & 0.9500 \\
$\mathrm{C}(11)-\mathrm{C}(16)$ & $1.379(4)$ \\
$\mathrm{C}(11)-\mathrm{C}(12)$ & $1.385(4)$ \\
$\mathrm{C}(12)-\mathrm{C}(13)$ & $1.387(4)$ \\
$\mathrm{C}(12)-\mathrm{H}(12)$ & 0.9500 \\
& \\
&
\end{tabular}


C(13)-C(14)

C(13)-H(13)

C(14)-C(15)

C(14)-C(17)

$\mathrm{C}(15)-\mathrm{C}(16)$

$\mathrm{C}(15)-\mathrm{H}(15)$

C(16)-H(16)

C(17)-H(17A)

C(17)-H(17B)

$\mathrm{C}(17)-\mathrm{H}(17 \mathrm{C})$

$\mathrm{C}(18)-\mathrm{C}(23)$

C(18)-C(19)

C(18)-H(18)

C(19)-C(20)

C(19)-H(19)

C(20)-C(21)

$\mathrm{C}(20)-\mathrm{C}(24)$

$\mathrm{C}(21)-\mathrm{C}(22)$

$\mathrm{C}(21)-\mathrm{H}(21)$

$\mathrm{C}(22)-\mathrm{C}(23)$

$\mathrm{C}(22)-\mathrm{H}(22)$

$\mathrm{C}(24)-\mathrm{H}(24 \mathrm{~A})$

$\mathrm{C}(24)-\mathrm{H}(24 \mathrm{~B})$

$\mathrm{C}(24)-\mathrm{H}(24 \mathrm{C})$

C(25)-C(26)

$\mathrm{C}(25)-\mathrm{H}(25)$

$\mathrm{C}(26)-\mathrm{H}(26)$

$\mathrm{C}(28)-\mathrm{C}(33)$

C(28)-C(29)

C(29)-C(30)

C(29)-H(29)

C(30)-C(31)

$\mathrm{C}(30)-\mathrm{H}(30)$

$\mathrm{C}(31)-\mathrm{C}(32)$

$\mathrm{C}(31)-\mathrm{C}(34)$

C(32)-C(33)

C(32)-H(32)

C(33)-H(33)

C(34)-H(34A)
1.389(4)

0.9500

1.386(4)

1.505(4)

1.390(4)

0.9500

0.9500

0.9800

0.9800

0.9800

1.383(4)

1.389(4)

0.9500

1.391(4)

0.9500

1.389(4)

$1.508(4)$

1.388(4)

0.9500

1.384(4)

0.9500

0.9800

0.9800

0.9800

$1.338(4)$

0.9500

0.9500

1.383(4)

1.389(4)

1.387(4)

0.9500

1.389(4)

0.9500

$1.396(4)$

1.509(4)

1.390(4)

0.9500

0.9500

0.9800 


\begin{tabular}{|c|c|}
\hline $\mathrm{C}(34)-\mathrm{H}(34 \mathrm{~B})$ & 0.9800 \\
\hline $\mathrm{C}(34)-\mathrm{H}(34 \mathrm{C})$ & 0.9800 \\
\hline $\mathrm{C}(27)-\mathrm{Ni}(1)-\mathrm{C}(10)$ & $176.78(11)$ \\
\hline $\mathrm{C}(27)-\mathrm{Ni}(1)-\mathrm{Cl}(2)$ & $89.24(8)$ \\
\hline $\mathrm{C}(10)-\mathrm{Ni}(1)-\mathrm{Cl}(2)$ & $93.93(8)$ \\
\hline $\mathrm{C}(27)-\mathrm{Ni}(1)-\mathrm{Cl}(1)$ & $89.55(8)$ \\
\hline $\mathrm{C}(10)-\mathrm{Ni}(1)-\mathrm{Cl}(1)$ & $87.29(8)$ \\
\hline $\mathrm{Cl}(2)-\mathrm{Ni}(1)-\mathrm{Cl}(1)$ & $178.30(3)$ \\
\hline $\mathrm{C}(10)-\mathrm{N}(1)-\mathrm{C}(8)$ & $111.6(2)$ \\
\hline$C(10)-N(1)-C(6)$ & $126.9(2)$ \\
\hline $\mathrm{C}(8)-\mathrm{N}(1)-\mathrm{C}(6)$ & $121.2(2)$ \\
\hline$C(10)-N(2)-C(9)$ & $111.7(2)$ \\
\hline $\mathrm{C}(10)-\mathrm{N}(2)-\mathrm{C}(11)$ & $126.1(2)$ \\
\hline $\mathrm{C}(9)-\mathrm{N}(2)-\mathrm{C}(11)$ & $122.1(2)$ \\
\hline $\mathrm{C}(27)-\mathrm{N}(3)-\mathrm{C}(25)$ & $111.8(2)$ \\
\hline $\mathrm{C}(27)-\mathrm{N}(3)-\mathrm{C}(23)$ & $126.0(2)$ \\
\hline$C(25)-N(3)-C(23)$ & $122.1(2)$ \\
\hline$C(27)-N(4)-C(26)$ & $111.3(2)$ \\
\hline $\mathrm{C}(27)-\mathrm{N}(4)-\mathrm{C}(28)$ & $126.7(2)$ \\
\hline$C(26)-N(4)-C(28)$ & $121.2(2)$ \\
\hline$C(6)-C(1)-C(2)$ & $119.6(3)$ \\
\hline $\mathrm{C}(6)-\mathrm{C}(1)-\mathrm{H}(1)$ & 120.2 \\
\hline $\mathrm{C}(2)-\mathrm{C}(1)-\mathrm{H}(1)$ & 120.2 \\
\hline$C(3)-C(2)-C(1)$ & $120.8(3)$ \\
\hline $\mathrm{C}(3)-\mathrm{C}(2)-\mathrm{H}(2)$ & 119.6 \\
\hline $\mathrm{C}(1)-\mathrm{C}(2)-\mathrm{H}(2)$ & 119.6 \\
\hline$C(2)-C(3)-C(4)$ & $118.4(3)$ \\
\hline$C(2)-C(3)-C(7)$ & $121.3(3)$ \\
\hline$C(4)-C(3)-C(7)$ & $120.3(3)$ \\
\hline$C(5)-C(4)-C(3)$ & $121.1(3)$ \\
\hline $\mathrm{C}(5)-\mathrm{C}(4)-\mathrm{H}(4)$ & 119.4 \\
\hline $\mathrm{C}(3)-\mathrm{C}(4)-\mathrm{H}(4)$ & 119.4 \\
\hline$C(6)-C(5)-C(4)$ & $118.9(3)$ \\
\hline $\mathrm{C}(6)-\mathrm{C}(5)-\mathrm{H}(5)$ & 120.6 \\
\hline $\mathrm{C}(4)-\mathrm{C}(5)-\mathrm{H}(5)$ & 120.6 \\
\hline$C(1)-C(6)-C(5)$ & $121.1(3)$ \\
\hline $\mathrm{C}(1)-\mathrm{C}(6)-\mathrm{N}(1)$ & $119.3(3)$ \\
\hline $\mathrm{C}(5)-\mathrm{C}(6)-\mathrm{N}(1)$ & $119.3(2)$ \\
\hline
\end{tabular}




\begin{tabular}{|c|c|}
\hline $\mathrm{C}(3)-\mathrm{C}(7)-\mathrm{H}(7 \mathrm{~A})$ & 109.5 \\
\hline $\mathrm{C}(3)-\mathrm{C}(7)-\mathrm{H}(7 \mathrm{~B})$ & 109.5 \\
\hline $\mathrm{H}(7 \mathrm{~A})-\mathrm{C}(7)-\mathrm{H}(7 \mathrm{~B})$ & 109.5 \\
\hline $\mathrm{C}(3)-\mathrm{C}(7)-\mathrm{H}(7 \mathrm{C})$ & 109.5 \\
\hline $\mathrm{H}(7 \mathrm{~A})-\mathrm{C}(7)-\mathrm{H}(7 \mathrm{C})$ & 109.5 \\
\hline H(7B)-C(7)-H(7C) & 109.5 \\
\hline $\mathrm{C}(9)-\mathrm{C}(8)-\mathrm{N}(1)$ & $106.8(2)$ \\
\hline $\mathrm{C}(9)-\mathrm{C}(8)-\mathrm{H}(8)$ & 126.6 \\
\hline $\mathrm{N}(1)-\mathrm{C}(8)-\mathrm{H}(8)$ & 126.6 \\
\hline $\mathrm{C}(8)-\mathrm{C}(9)-\mathrm{N}(2)$ & $106.6(2)$ \\
\hline $\mathrm{C}(8)-\mathrm{C}(9)-\mathrm{H}(9)$ & 126.7 \\
\hline $\mathrm{N}(2)-\mathrm{C}(9)-\mathrm{H}(9)$ & 126.7 \\
\hline $\mathrm{N}(1)-\mathrm{C}(10)-\mathrm{N}(2)$ & $103.3(2)$ \\
\hline $\mathrm{N}(1)-\mathrm{C}(10)-\mathrm{Ni}(1)$ & $127.67(19)$ \\
\hline $\mathrm{N}(2)-\mathrm{C}(10)-\mathrm{Ni}(1)$ & $127.85(19)$ \\
\hline$C(16)-C(11)-C(12)$ & $120.2(3)$ \\
\hline $\mathrm{C}(16)-\mathrm{C}(11)-\mathrm{N}(2)$ & $120.0(3)$ \\
\hline $\mathrm{C}(12)-\mathrm{C}(11)-\mathrm{N}(2)$ & $119.7(2)$ \\
\hline $\mathrm{C}(11)-\mathrm{C}(12)-\mathrm{C}(13)$ & $118.8(3)$ \\
\hline $\mathrm{C}(11)-\mathrm{C}(12)-\mathrm{H}(12)$ & 120.6 \\
\hline $\mathrm{C}(13)-\mathrm{C}(12)-\mathrm{H}(12)$ & 120.6 \\
\hline$C(12)-C(13)-C(14)$ & $122.4(3)$ \\
\hline $\mathrm{C}(12)-\mathrm{C}(13)-\mathrm{H}(13)$ & 118.8 \\
\hline $\mathrm{C}(14)-\mathrm{C}(13)-\mathrm{H}(13)$ & 118.8 \\
\hline $\mathrm{C}(15)-\mathrm{C}(14)-\mathrm{C}(13)$ & $117.4(3)$ \\
\hline $\mathrm{C}(15)-\mathrm{C}(14)-\mathrm{C}(17)$ & $120.5(3)$ \\
\hline $\mathrm{C}(13)-\mathrm{C}(14)-\mathrm{C}(17)$ & $122.1(3)$ \\
\hline $\mathrm{C}(14)-\mathrm{C}(15)-\mathrm{C}(16)$ & $121.3(3)$ \\
\hline $\mathrm{C}(14)-\mathrm{C}(15)-\mathrm{H}(15)$ & 119.4 \\
\hline $\mathrm{C}(16)-\mathrm{C}(15)-\mathrm{H}(15)$ & 119.4 \\
\hline $\mathrm{C}(11)-\mathrm{C}(16)-\mathrm{C}(15)$ & $120.0(3)$ \\
\hline $\mathrm{C}(11)-\mathrm{C}(16)-\mathrm{H}(16)$ & 120.0 \\
\hline $\mathrm{C}(15)-\mathrm{C}(16)-\mathrm{H}(16)$ & 120.0 \\
\hline $\mathrm{C}(14)-\mathrm{C}(17)-\mathrm{H}(17 \mathrm{~A})$ & 109.5 \\
\hline $\mathrm{C}(14)-\mathrm{C}(17)-\mathrm{H}(17 \mathrm{~B})$ & 109.5 \\
\hline $\mathrm{H}(17 \mathrm{~A})-\mathrm{C}(17)-\mathrm{H}(17 \mathrm{~B})$ & 109.5 \\
\hline $\mathrm{C}(14)-\mathrm{C}(17)-\mathrm{H}(17 \mathrm{C})$ & 109.5 \\
\hline $\mathrm{H}(17 \mathrm{~A})-\mathrm{C}(17)-\mathrm{H}(17 \mathrm{C})$ & 109.5 \\
\hline $\mathrm{H}(17 \mathrm{~B})-\mathrm{C}(17)-\mathrm{H}(17 \mathrm{C})$ & 109.5 \\
\hline
\end{tabular}


C(23)-C(18)-C(19)

C(23)-C(18)-H(18)

C(19)-C(18)-H(18)

C(18)-C(19)-C(20)

C(18)-C(19)-H(19)

C(20)-C(19)-H(19)

$\mathrm{C}(21)-\mathrm{C}(20)-\mathrm{C}(19)$

$\mathrm{C}(21)-\mathrm{C}(20)-\mathrm{C}(24)$

$\mathrm{C}(19)-\mathrm{C}(20)-\mathrm{C}(24)$

$\mathrm{C}(22)-\mathrm{C}(21)-\mathrm{C}(20)$

$\mathrm{C}(22)-\mathrm{C}(21)-\mathrm{H}(21)$

$\mathrm{C}(20)-\mathrm{C}(21)-\mathrm{H}(21)$

$\mathrm{C}(23)-\mathrm{C}(22)-\mathrm{C}(21)$

$\mathrm{C}(23)-\mathrm{C}(22)-\mathrm{H}(22)$

$\mathrm{C}(21)-\mathrm{C}(22)-\mathrm{H}(22)$

$\mathrm{C}(18)-\mathrm{C}(23)-\mathrm{C}(22)$

$\mathrm{C}(18)-\mathrm{C}(23)-\mathrm{N}(3)$

$\mathrm{C}(22)-\mathrm{C}(23)-\mathrm{N}(3)$

$\mathrm{C}(20)-\mathrm{C}(24)-\mathrm{H}(24 \mathrm{~A})$

$\mathrm{C}(20)-\mathrm{C}(24)-\mathrm{H}(24 \mathrm{~B})$

$\mathrm{H}(24 \mathrm{~A})-\mathrm{C}(24)-\mathrm{H}(24 \mathrm{~B})$

$\mathrm{C}(20)-\mathrm{C}(24)-\mathrm{H}(24 \mathrm{C})$

$\mathrm{H}(24 \mathrm{~A})-\mathrm{C}(24)-\mathrm{H}(24 \mathrm{C})$

$\mathrm{H}(24 \mathrm{~B})-\mathrm{C}(24)-\mathrm{H}(24 \mathrm{C})$

$\mathrm{C}(26)-\mathrm{C}(25)-\mathrm{N}(3)$

$\mathrm{C}(26)-\mathrm{C}(25)-\mathrm{H}(25)$

$\mathrm{N}(3)-\mathrm{C}(25)-\mathrm{H}(25)$

$\mathrm{C}(25)-\mathrm{C}(26)-\mathrm{N}(4)$

$\mathrm{C}(25)-\mathrm{C}(26)-\mathrm{H}(26)$

$\mathrm{N}(4)-\mathrm{C}(26)-\mathrm{H}(26)$

$\mathrm{N}(3)-\mathrm{C}(27)-\mathrm{N}(4)$

$\mathrm{N}(3)-\mathrm{C}(27)-\mathrm{Ni}(1)$

$\mathrm{N}(4)-\mathrm{C}(27)-\mathrm{Ni}(1)$

$\mathrm{C}(33)-\mathrm{C}(28)-\mathrm{C}(29)$

$\mathrm{C}(33)-\mathrm{C}(28)-\mathrm{N}(4)$

$\mathrm{C}(29)-\mathrm{C}(28)-\mathrm{N}(4)$

$\mathrm{C}(30)-\mathrm{C}(29)-\mathrm{C}(28)$

$\mathrm{C}(30)-\mathrm{C}(29)-\mathrm{H}(29)$

C(28)-C(29)-H(29)
119.2(3)

120.4

120.4

121.6(3)

119.2

119.2

117.9(2)

121.5(3)

120.6(3)

121.3(3)

119.4

119.4

119.6(3)

120.2

120.2

120.4(2)

120.6(2)

118.9(2)

109.5

109.5

109.5

109.5

109.5

109.5

106.4(2)

126.8

126.8

107.2(2)

126.4

126.4

103.4(2)

127.72(18)

128.91(19)

120.4(2)

120.5(2)

118.8(2)

119.5(3)

120.3

120.3 


$\begin{array}{ll}\mathrm{C}(29)-\mathrm{C}(30)-\mathrm{C}(31) & 121.6(3) \\ \mathrm{C}(29)-\mathrm{C}(30)-\mathrm{H}(30) & 119.2 \\ \mathrm{C}(31)-\mathrm{C}(30)-\mathrm{H}(30) & 119.2 \\ \mathrm{C}(30)-\mathrm{C}(31)-\mathrm{C}(32) & 117.6(3) \\ \mathrm{C}(30)-\mathrm{C}(31)-\mathrm{C}(34) & 121.5(3) \\ \mathrm{C}(32)-\mathrm{C}(31)-\mathrm{C}(34) & 120.9(3) \\ \mathrm{C}(33)-\mathrm{C}(32)-\mathrm{C}(31) & 121.8(3) \\ \mathrm{C}(33)-\mathrm{C}(32)-\mathrm{H}(32) & 119.1 \\ \mathrm{C}(31)-\mathrm{C}(32)-\mathrm{H}(32) & 119.1 \\ \mathrm{C}(28)-\mathrm{C}(33)-\mathrm{C}(32) & 119.1(2) \\ \mathrm{C}(28)-\mathrm{C}(33)-\mathrm{H}(33) & 120.5 \\ \mathrm{C}(32)-\mathrm{C}(33)-\mathrm{H}(33) & 120.5 \\ \mathrm{C}(31)-\mathrm{C}(34)-\mathrm{H}(34 \mathrm{~A}) & 109.5 \\ \mathrm{C}(31)-\mathrm{C}(34)-\mathrm{H}(34 \mathrm{~B}) & 109.5 \\ \mathrm{H}(34 \mathrm{~A})-\mathrm{C}(34)-\mathrm{H}(34 \mathrm{~B}) & 109.5 \\ \mathrm{C}(31)-\mathrm{C}(34)-\mathrm{H}(34 \mathrm{C}) & 109.5 \\ \mathrm{H}(34 \mathrm{~A})-\mathrm{C}(34)-\mathrm{H}(34 \mathrm{C}) & 109.5 \\ \mathrm{H}(34 \mathrm{~B})-\mathrm{C}(34)-\mathrm{H}(34 \mathrm{C}) & 109.5\end{array}$

Symmetry transformations used to generate equivalent atoms: 
Table S-22. Anisotropic displacement parameters $\left(\AA^{2} \times 10^{3}\right)$ for trans-[ITol $\left.{ }_{2} \mathrm{NiCl}_{2}\right](3 \mathbf{h})$. The anisotropic displacement factor exponent takes the form: $-2 \pi^{2}\left[h^{2} a^{* 2} U^{11}+\ldots+2 h k a^{*} b^{*} U^{12}\right]$

\begin{tabular}{|c|c|c|c|c|c|}
\hline & U11 & U22 & U33 & U23 & U13 \\
\hline $\mathrm{Ni}(1)$ & $22(1)$ & $22(1)$ & $19(1)$ & 1(1) & $7(1)$ \\
\hline $\mathrm{Cl}(1)$ & $30(1)$ & $26(1)$ & $34(1)$ & 1(1) & 15(1) \\
\hline $\mathrm{Cl}(2)$ & $31(1)$ & $26(1)$ & $28(1)$ & $4(1)$ & $10(1)$ \\
\hline $\mathrm{N}(1)$ & $29(1)$ & $25(1)$ & $22(1)$ & $0(1)$ & $10(1)$ \\
\hline $\mathrm{N}(2)$ & $29(1)$ & $27(1)$ & $20(1)$ & $1(1)$ & $7(1)$ \\
\hline $\mathrm{N}(3)$ & $27(1)$ & $26(1)$ & $22(1)$ & 2(1) & $9(1)$ \\
\hline $\mathrm{N}(4)$ & $26(1)$ & $26(1)$ & $21(1)$ & $0(1)$ & $10(1)$ \\
\hline $\mathrm{C}(1)$ & $31(2)$ & $28(2)$ & $37(2)$ & $4(1)$ & $14(1)$ \\
\hline$C(2)$ & $32(2)$ & $38(2)$ & $42(2)$ & $-1(1)$ & $15(1)$ \\
\hline$C(3)$ & $32(2)$ & $36(2)$ & $30(2)$ & $-1(1)$ & $10(1)$ \\
\hline $\mathrm{C}(4)$ & $37(2)$ & $32(2)$ & $31(2)$ & $6(1)$ & 14(1) \\
\hline$C(5)$ & $31(1)$ & $32(2)$ & $28(2)$ & $0(1)$ & 12(1) \\
\hline$C(6)$ & $32(1)$ & $27(1)$ & $22(1)$ & $-3(1)$ & $9(1)$ \\
\hline$C(7)$ & $38(2)$ & $51(2)$ & $51(2)$ & $8(2)$ & $13(2)$ \\
\hline $\mathrm{C}(8)$ & $40(2)$ & $32(2)$ & $25(2)$ & $-1(1)$ & $16(1)$ \\
\hline C(9) & $40(2)$ & $33(2)$ & $20(1)$ & $1(1)$ & 11(1) \\
\hline$C(10)$ & $24(1)$ & 21(1) & $24(1)$ & 2(1) & $8(1)$ \\
\hline $\mathrm{C}(11)$ & $32(1)$ & $29(2)$ & $20(1)$ & 2(1) & $6(1)$ \\
\hline $\mathrm{C}(12)$ & $30(1)$ & $32(2)$ & $26(2)$ & $8(1)$ & $7(1)$ \\
\hline$C(13)$ & $32(2)$ & $35(2)$ & $35(2)$ & $4(1)$ & $12(1)$ \\
\hline$C(14)$ & $33(2)$ & $32(2)$ & $30(2)$ & $0(1)$ & $5(1)$ \\
\hline$C(15)$ & $47(2)$ & $35(2)$ & $33(2)$ & $12(1)$ & 13(1) \\
\hline$C(16)$ & $40(2)$ & $39(2)$ & $35(2)$ & $13(1)$ & 17(1) \\
\hline $\mathrm{C}(17)$ & $39(2)$ & $43(2)$ & $55(2)$ & $4(2)$ & $10(2)$ \\
\hline$C(18)$ & $28(1)$ & $30(2)$ & $24(1)$ & $-1(1)$ & $5(1)$ \\
\hline$C(19)$ & $37(2)$ & $28(2)$ & $28(2)$ & $0(1)$ & 11(1) \\
\hline$C(20)$ & $29(1)$ & $31(2)$ & $26(1)$ & $7(1)$ & $10(1)$ \\
\hline$C(21)$ & $29(1)$ & $29(2)$ & $29(2)$ & 2(1) & $5(1)$ \\
\hline$C(22)$ & $33(2)$ & $24(1)$ & $30(2)$ & 1(1) & $7(1)$ \\
\hline$C(23)$ & $26(1)$ & $26(1)$ & $20(1)$ & $4(1)$ & $6(1)$ \\
\hline$C(24)$ & $33(2)$ & $41(2)$ & $44(2)$ & $13(1)$ & $13(1)$ \\
\hline$C(25)$ & $37(2)$ & $32(2)$ & $21(1)$ & $1(1)$ & $12(1)$ \\
\hline$C(26)$ & $34(2)$ & $32(2)$ & $23(1)$ & $1(1)$ & $12(1)$ \\
\hline \multirow[t]{2}{*}{$C(27)$} & $24(1)$ & $20(1)$ & $22(1)$ & $3(1)$ & $9(1)$ \\
\hline & & & S-73 & & \\
\hline
\end{tabular}




$\begin{array}{llllll}\mathrm{C}(28) & 22(1) & 21(1) & 26(1) & -1(1) & 7(1) \\ \mathrm{C}(29) & 32(1) & 31(2) & 29(2) & 2(1) & 14(1) \\ \mathrm{C}(30) & 26(1) & 34(2) & 36(2) & -2(1) & 12(1) \\ \mathrm{C}(31) & 30(1) & 33(2) & 32(2) & -3(1) & 7(1) \\ \mathrm{C}(32) & 30(1) & 30(2) & 29(2) & 3(1) & 11(1) \\ \mathrm{C}(33) & 26(1) & 26(1) & 28(2) & 0(1) & 10(1) \\ \mathrm{C}(34) & 37(2) & 65(2) & 42(2) & 10(2) & 10(2)\end{array}$


Table S-23. Hydrogen coordinates ( $\left.\times 10^{4}\right)$ and isotropic displacement parameters $\left(\AA^{2} \times 10^{3}\right)$ for trans-[ITol $\left.{ }_{2} \mathrm{NiCl}_{2}\right]$ (3h).

\begin{tabular}{|c|c|c|c|c|}
\hline & $\mathrm{x}$ & $\mathrm{y}$ & z & $\mathrm{U}(\mathrm{eq})$ \\
\hline $\mathrm{H}(1)$ & 6180 & 1919 & 1588 & 38 \\
\hline $\mathrm{H}(2)$ & 8015 & 1089 & 2655 & 44 \\
\hline $\mathrm{H}(4)$ & 4928 & -1178 & 3279 & 39 \\
\hline $\mathrm{H}(5)$ & 3082 & -368 & 2183 & 36 \\
\hline $\mathrm{H}(7 \mathrm{~A})$ & 7873 & -668 & 4494 & 69 \\
\hline $\mathrm{H}(7 \mathrm{~B})$ & 8796 & -307 & 3741 & 69 \\
\hline $\mathrm{H}(7 \mathrm{C})$ & 7591 & -1475 & 3431 & 69 \\
\hline $\mathrm{H}(8)$ & 3630 & 781 & -470 & 38 \\
\hline $\mathrm{H}(9)$ & 1371 & 1406 & -1452 & 37 \\
\hline $\mathrm{H}(12)$ & -849 & 1117 & 733 & 37 \\
\hline $\mathrm{H}(13)$ & -3080 & 1633 & 158 & 42 \\
\hline $\mathrm{H}(15)$ & -1720 & 3771 & -1705 & 45 \\
\hline $\mathrm{H}(16)$ & 514 & 3259 & -1149 & 44 \\
\hline $\mathrm{H}(17 \mathrm{~A})$ & -3928 & 3858 & -1370 & 70 \\
\hline $\mathrm{H}(17 \mathrm{~B})$ & -4488 & 3010 & -634 & 70 \\
\hline $\mathrm{H}(17 \mathrm{C})$ & -4790 & 2598 & -1876 & 70 \\
\hline $\mathrm{H}(18)$ & 4152 & 1328 & 4295 & 34 \\
\hline $\mathrm{H}(19)$ & 6393 & 822 & 4816 & 37 \\
\hline $\mathrm{H}(21)$ & 8577 & 3899 & 6397 & 37 \\
\hline $\mathrm{H}(22)$ & 6340 & 4399 & 5927 & 36 \\
\hline $\mathrm{H}(24 \mathrm{~A})$ & 9969 & 2579 & 6465 & 57 \\
\hline $\mathrm{H}(24 \mathrm{~B})$ & 9335 & 1773 & 5339 & 57 \\
\hline $\mathrm{H}(24 \mathrm{C})$ & 9101 & 1315 & 6398 & 57 \\
\hline $\mathrm{H}(25)$ & 4014 & 3975 & 6401 & 35 \\
\hline $\mathrm{H}(26)$ & 1524 & 4289 & 5489 & 35 \\
\hline $\mathrm{H}(29)$ & -1027 & 3409 & 4201 & 36 \\
\hline $\mathrm{H}(30)$ & -3004 & 4042 & 3047 & 38 \\
\hline $\mathrm{H}(32)$ & -991 & 4638 & 905 & 35 \\
\hline $\mathrm{H}(33)$ & 1009 & 4022 & 2059 & 32 \\
\hline $\mathrm{H}(34 \mathrm{~A})$ & -4354 & 4441 & 1017 & 71 \\
\hline $\mathrm{H}(34 \mathrm{~B})$ & -3264 & 5113 & 491 & 71 \\
\hline $\mathrm{H}(34 \mathrm{C})$ & -3347 & 5657 & 1571 & 71 \\
\hline
\end{tabular}


TableS-24. Torsion angles $\left[^{\circ}\right]$ for trans-[$\left[\mathrm{TOO}_{2} \mathrm{NiCl}_{2}\right](3 \mathbf{h})$.

\begin{tabular}{ll}
\hline $\mathrm{C}(6)-\mathrm{C}(1)-\mathrm{C}(2)-\mathrm{C}(3)$ & $-0.8(4)$ \\
$\mathrm{C}(1)-\mathrm{C}(2)-\mathrm{C}(3)-\mathrm{C}(4)$ & $-1.8(4)$ \\
$\mathrm{C}(1)-\mathrm{C}(2)-\mathrm{C}(3)-\mathrm{C}(7)$ & $179.2(3)$ \\
$\mathrm{C}(2)-\mathrm{C}(3)-\mathrm{C}(4)-\mathrm{C}(5)$ & $2.4(4)$ \\
$\mathrm{C}(7)-\mathrm{C}(3)-\mathrm{C}(4)-\mathrm{C}(5)$ & $-178.6(3)$ \\
$\mathrm{C}(3)-\mathrm{C}(4)-\mathrm{C}(5)-\mathrm{C}(6)$ & $-0.5(4)$ \\
$\mathrm{C}(2)-\mathrm{C}(1)-\mathrm{C}(6)-\mathrm{C}(5)$ & $2.8(4)$ \\
$\mathrm{C}(2)-\mathrm{C}(1)-\mathrm{C}(6)-\mathrm{N}(1)$ & $-170.0(2)$ \\
$\mathrm{C}(4)-\mathrm{C}(5)-\mathrm{C}(6)-\mathrm{C}(1)$ & $-2.1(4)$ \\
$\mathrm{C}(4)-\mathrm{C}(5)-\mathrm{C}(6)-\mathrm{N}(1)$ & $170.6(2)$ \\
$\mathrm{C}(10)-\mathrm{N}(1)-\mathrm{C}(6)-\mathrm{C}(1)$ & $-122.3(3)$ \\
$\mathrm{C}(8)-\mathrm{N}(1)-\mathrm{C}(6)-\mathrm{C}(1)$ & $64.7(3)$ \\
$\mathrm{C}(10)-\mathrm{N}(1)-\mathrm{C}(6)-\mathrm{C}(5)$ & $64.7(4)$ \\
$\mathrm{C}(8)-\mathrm{N}(1)-\mathrm{C}(6)-\mathrm{C}(5)$ & $-108.2(3)$ \\
$\mathrm{C}(10)-\mathrm{N}(1)-\mathrm{C}(8)-\mathrm{C}(9)$ & $-1.1(3)$ \\
$\mathrm{C}(6)-\mathrm{N}(1)-\mathrm{C}(8)-\mathrm{C}(9)$ & $172.9(3)$ \\
$\mathrm{N}(1)-\mathrm{C}(8)-\mathrm{C}(9)-\mathrm{N}(2)$ & $0.0(3)$ \\
$\mathrm{C}(10)-\mathrm{N}(2)-\mathrm{C}(9)-\mathrm{C}(8)$ & $1.1(3)$ \\
$\mathrm{C}(11)-\mathrm{N}(2)-\mathrm{C}(9)-\mathrm{C}(8)$ & $-176.9(2)$ \\
$\mathrm{C}(8)-\mathrm{N}(1)-\mathrm{C}(10)-\mathrm{N}(2)$ & $1.7(3)$ \\
$\mathrm{C}(6)-\mathrm{N}(1)-\mathrm{C}(10)-\mathrm{N}(2)$ & $-171.9(2)$ \\
$\mathrm{C}(8)-\mathrm{N}(1)-\mathrm{C}(10)-\mathrm{Ni}(1)$ & $-166.5(2)$ \\
$\mathrm{C}(6)-\mathrm{N}(1)-\mathrm{C}(10)-\mathrm{Ni}(1)$ & $20.0(4)$ \\
$\mathrm{C}(9)-\mathrm{N}(2)-\mathrm{C}(10)-\mathrm{N}(1)$ & $-1.7(3)$ \\
$\mathrm{C}(11)-\mathrm{N}(2)-\mathrm{C}(10)-\mathrm{N}(1)$ & $176.3(2)$ \\
$\mathrm{C}(9)-\mathrm{N}(2)-\mathrm{C}(10)-\mathrm{Ni}(1)$ & $166.4(2)$ \\
$\mathrm{C}(11)-\mathrm{N}(2)-\mathrm{C}(10)-\mathrm{Ni}(1)$ & $-15.6(4)$ \\
$\mathrm{C}(27)-\mathrm{Ni}(1)-\mathrm{C}(10)-\mathrm{N}(1)$ & $92.5(18)$ \\
$\mathrm{Cl}(2)-\mathrm{Ni}(1)-\mathrm{C}(10)-\mathrm{N}(1)$ & $-96.6(2)$ \\
$\mathrm{Cl}(1)-\mathrm{Ni}(1)-\mathrm{C}(10)-\mathrm{N}(1)$ & $82.2(2)$ \\
$\mathrm{C}(27)-\mathrm{Ni}(1)-\mathrm{C}(10)-\mathrm{N}(2)$ & $-72.9(19)$ \\
$\mathrm{Cl}(2)-\mathrm{Ni}(1)-\mathrm{C}(10)-\mathrm{N}(2)$ & $98.1(2)$ \\
$\mathrm{Cl}(1)-\mathrm{Ni}(1)-\mathrm{C}(10)-\mathrm{N}(2)$ & $-83.1(2)$ \\
$\mathrm{C}(10)-\mathrm{N}(2)-\mathrm{C}(11)-\mathrm{C}(16)$ & $132.0(3)$ \\
$\mathrm{C}(9)-\mathrm{N}(2)-\mathrm{C}(11)-\mathrm{C}(16)$ & $-50.3(4)$ \\
$\mathrm{C}(10)-\mathrm{N}(2)-\mathrm{C}(11)-\mathrm{C}(12)$ & \\
$\mathrm{C}(9)-\mathrm{N}(2)-\mathrm{C}(11)-\mathrm{C}(12)$ & \\
& \\
& \\
&
\end{tabular}




\begin{tabular}{|c|c|}
\hline$C(16)-C(11)-C(12)-C(13)$ & $0.7(4)$ \\
\hline $\mathrm{N}(2)-\mathrm{C}(11)-\mathrm{C}(12)-\mathrm{C}(13)$ & $-175.3(2)$ \\
\hline$C(11)-C(12)-C(13)-C(14)$ & $-0.5(5)$ \\
\hline $\mathrm{C}(12)-\mathrm{C}(13)-\mathrm{C}(14)-\mathrm{C}(15)$ & $0.2(5)$ \\
\hline$C(12)-C(13)-C(14)-C(17)$ & $179.3(3)$ \\
\hline $\mathrm{C}(13)-\mathrm{C}(14)-\mathrm{C}(15)-\mathrm{C}(16)$ & $0.0(5)$ \\
\hline$C(17)-C(14)-C(15)-C(16)$ & $-179.0(3)$ \\
\hline $\mathrm{C}(12)-\mathrm{C}(11)-\mathrm{C}(16)-\mathrm{C}(15)$ & $-0.5(5)$ \\
\hline $\mathrm{N}(2)-\mathrm{C}(11)-\mathrm{C}(16)-\mathrm{C}(15)$ & $175.5(3)$ \\
\hline $\mathrm{C}(14)-\mathrm{C}(15)-\mathrm{C}(16)-\mathrm{C}(11)$ & $0.1(5)$ \\
\hline$C(23)-C(18)-C(19)-C(20)$ & $-1.4(4)$ \\
\hline $\mathrm{C}(18)-\mathrm{C}(19)-\mathrm{C}(20)-\mathrm{C}(21)$ & $0.1(4)$ \\
\hline$C(18)-C(19)-C(20)-C(24)$ & $179.2(3)$ \\
\hline$C(19)-C(20)-C(21)-C(22)$ & $0.9(4)$ \\
\hline$C(24)-C(20)-C(21)-C(22)$ & $-178.2(3)$ \\
\hline $\mathrm{C}(20)-\mathrm{C}(21)-\mathrm{C}(22)-\mathrm{C}(23)$ & $-0.6(4)$ \\
\hline$C(19)-C(18)-C(23)-C(22)$ & $1.7(4)$ \\
\hline $\mathrm{C}(19)-\mathrm{C}(18)-\mathrm{C}(23)-\mathrm{N}(3)$ & $-175.5(3)$ \\
\hline $\mathrm{C}(21)-\mathrm{C}(22)-\mathrm{C}(23)-\mathrm{C}(18)$ & $-0.7(4)$ \\
\hline $\mathrm{C}(21)-\mathrm{C}(22)-\mathrm{C}(23)-\mathrm{N}(3)$ & $176.5(3)$ \\
\hline $\mathrm{C}(27)-\mathrm{N}(3)-\mathrm{C}(23)-\mathrm{C}(18)$ & $-48.5(4)$ \\
\hline $\mathrm{C}(25)-\mathrm{N}(3)-\mathrm{C}(23)-\mathrm{C}(18)$ & $132.9(3)$ \\
\hline $\mathrm{C}(27)-\mathrm{N}(3)-\mathrm{C}(23)-\mathrm{C}(22)$ & $134.2(3)$ \\
\hline $\mathrm{C}(25)-\mathrm{N}(3)-\mathrm{C}(23)-\mathrm{C}(22)$ & $-44.4(4)$ \\
\hline $\mathrm{C}(27)-\mathrm{N}(3)-\mathrm{C}(25)-\mathrm{C}(26)$ & $0.1(3)$ \\
\hline $\mathrm{C}(23)-\mathrm{N}(3)-\mathrm{C}(25)-\mathrm{C}(26)$ & $178.9(2)$ \\
\hline $\mathrm{N}(3)-\mathrm{C}(25)-\mathrm{C}(26)-\mathrm{N}(4)$ & $-0.3(3)$ \\
\hline $\mathrm{C}(27)-\mathrm{N}(4)-\mathrm{C}(26)-\mathrm{C}(25)$ & $0.4(3)$ \\
\hline $\mathrm{C}(28)-\mathrm{N}(4)-\mathrm{C}(26)-\mathrm{C}(25)$ & $-169.3(2)$ \\
\hline $\mathrm{C}(25)-\mathrm{N}(3)-\mathrm{C}(27)-\mathrm{N}(4)$ & $0.1(3)$ \\
\hline $\mathrm{C}(23)-\mathrm{N}(3)-\mathrm{C}(27)-\mathrm{N}(4)$ & $-178.6(2)$ \\
\hline $\mathrm{C}(25)-\mathrm{N}(3)-\mathrm{C}(27)-\mathrm{Ni}(1)$ & $-179.9(2)$ \\
\hline $\mathrm{C}(23)-\mathrm{N}(3)-\mathrm{C}(27)-\mathrm{Ni}(1)$ & $1.4(4)$ \\
\hline $\mathrm{C}(26)-\mathrm{N}(4)-\mathrm{C}(27)-\mathrm{N}(3)$ & $-0.3(3)$ \\
\hline $\mathrm{C}(28)-\mathrm{N}(4)-\mathrm{C}(27)-\mathrm{N}(3)$ & $168.6(2)$ \\
\hline $\mathrm{C}(26)-\mathrm{N}(4)-\mathrm{C}(27)-\mathrm{Ni}(1)$ & $179.7(2)$ \\
\hline $\mathrm{C}(28)-\mathrm{N}(4)-\mathrm{C}(27)-\mathrm{Ni}(1)$ & $-11.3(4)$ \\
\hline $\mathrm{C}(10)-\mathrm{Ni}(1)-\mathrm{C}(27)-\mathrm{N}(3)$ & $-90.0(18)$ \\
\hline $\mathrm{Cl}(2)-\mathrm{Ni}(1)-\mathrm{C}(27)-\mathrm{N}(3)$ & $99.1(2)$ \\
\hline
\end{tabular}




$\begin{array}{ll}\mathrm{Cl}(1)-\mathrm{Ni}(1)-\mathrm{C}(27)-\mathrm{N}(3) & -79.8(2) \\ \mathrm{C}(10)-\mathrm{Ni}(1)-\mathrm{C}(27)-\mathrm{N}(4) & 90.0(18) \\ \mathrm{Cl}(2)-\mathrm{Ni}(1)-\mathrm{C}(27)-\mathrm{N}(4) & -81.0(2) \\ \mathrm{Cl}(1)-\mathrm{Ni}(1)-\mathrm{C}(27)-\mathrm{N}(4) & 100.2(2) \\ \mathrm{C}(27)-\mathrm{N}(4)-\mathrm{C}(28)-\mathrm{C}(33) & -35.9(4) \\ \mathrm{C}(26)-\mathrm{N}(4)-\mathrm{C}(28)-\mathrm{C}(33) & 132.1(3) \\ \mathrm{C}(27)-\mathrm{N}(4)-\mathrm{C}(28)-\mathrm{C}(29) & 150.8(3) \\ \mathrm{C}(26)-\mathrm{N}(4)-\mathrm{C}(28)-\mathrm{C}(29) & -41.3(4) \\ \mathrm{C}(33)-\mathrm{C}(28)-\mathrm{C}(29)-\mathrm{C}(30) & -3.7(4) \\ \mathrm{N}(4)-\mathrm{C}(28)-\mathrm{C}(29)-\mathrm{C}(30) & 169.6(2) \\ \mathrm{C}(28)-\mathrm{C}(29)-\mathrm{C}(30)-\mathrm{C}(31) & 0.2(4) \\ \mathrm{C}(29)-\mathrm{C}(30)-\mathrm{C}(31)-\mathrm{C}(32) & 3.5(4) \\ \mathrm{C}(29)-\mathrm{C}(30)-\mathrm{C}(31)-\mathrm{C}(34) & -174.5(3) \\ \mathrm{C}(30)-\mathrm{C}(31)-\mathrm{C}(32)-\mathrm{C}(33) & -3.9(4) \\ \mathrm{C}(34)-\mathrm{C}(31)-\mathrm{C}(32)-\mathrm{C}(33) & 174.2(3) \\ \mathrm{C}(29)-\mathrm{C}(28)-\mathrm{C}(33)-\mathrm{C}(32) & 3.4(4) \\ \mathrm{N}(4)-\mathrm{C}(28)-\mathrm{C}(33)-\mathrm{C}(32) & -169.8(2) \\ \mathrm{C}(31)-\mathrm{C}(32)-\mathrm{C}(33)-\mathrm{C}(28) & 0.4(4)\end{array}$

Symmetry transformations used to generate equivalent atoms: 